\title{
Investigation of SNARE function in the early endosomal compartment
}

\author{
PhD Thesis \\ in partial fulfilment of the requirements for the degree \\ Doctor of Philosophy (PhD) in the Neuroscience Program \\ at the Georg August University Göttingen, Faculty of Biology
}

submitted by

Ioanna Bethani

born in

Athens, Greece

March 2009 

Herewith I declare, that I prepared the PhD Thesis 'Investigation of SNARE function in the early endosomal compartment' on my own and with no other sources and aids than quoted.

Göttingen, March $18^{\text {th }} 2009$. 

- The cell has a memory of its past, certainly in the case of the egg cell, and foresight of the future, together with precise and detailed patterns for differentiations and growth, a knowledge which is materialized in the process of reproduction and the development of all beings from bacteria to plants, beasts, or men. It is this cell which plans and composes all organisms, and which transmits to them its defects and potentialities. Man, like other organisms, is so perfectly coordinated that he may easily forget, whether awake or asleep, that he is a colony of cells in action, and that it is the cells which achieve, through him, what he has the illusion of accomplishing himself. It is the cells which create and maintain in us, during the span of our lives, our will to live and survive, to search and experiment, and to struggle.

Albert Claude

Nobel Lecture, December 12, 1974 



\section{Contents}

Table of Contents vii

List of Figures $\quad$ xi

List of Tables $\quad$ xiii

$\begin{array}{ll}\text { Abstract } & \text { XV }\end{array}$

$\begin{array}{ll}\text { Acknowledgments } & \text { xvi }\end{array}$

1 Introduction 1

1.1 SNAREs: key players of membrane fusion . . . . . . . . . . . . 2

1.1.1 SNARE structure . . . . . . . . . . . . . . . . 4

1.1.2 SNARE function and its regulation in membrane fusion . . . . 6

1.1.3 Specificity in SNARE function: a controversial issue . . . . . . 9

1.2 Early endosomes and endosomal trafficking . . . . . . . . . . . . . 10

1.2.1 Endocytosis and early endosomes . . . . . . . . . . . . 10

1.2.2 Characteristics of homotypic early endosomal fusion: the SNAREs involved . . . . . . . . . . . . . . 13

1.2.3 Promiscuous SNARE associations on the endosomal membrane 15

1.3 Aims of this study . . . . . . . . . . . . . . . . . . 18

2 Materials and Methods $\quad 19$

2.1 Materials ............................. 19

2.1.1 Chemicals ..................... 19 
2.1.2 Enzymes, kits and bacterial strains . . . . . . . . . . . 19

2.1.3 siRNA and shRNA sequences . . . . . . . . . . . . . 19

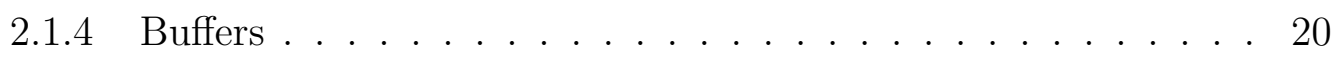

2.1.5 Antibodies ..................... . . 21

2.2 Methods . . . . . . . . . . . . . . . . . . . . 21

2.2.1 Cell culture . . . . . . . . . . . . . . . . . . . 21

2.2.2 Transfection of DNA plasmids and siRNA oligonucleotides . . 22

2.2.3 Preparation of postnuclear supernatant from PC12 cells . . . . 23

2.2.4 Purification of early endosomes from PC12 cells via density gradient centrifugation . . . . . . . . . . . 23

2.2.5 Preparation of synaptic-vesicle-enriched LS1 fraction and cytosol from rat brains . . . . . . . . . . . . . . 24

2.2.6 Preparation of rat brain cytosol . . . . . . . . . . 25

2.2.7 Cell-free fusion and docking assay . . . . . . . . . . 25

2.2 .8 Immunoprecipitation . . . . . . . . . . . . . . . . 26

2.2.9 Western blotting . . . . . . . . . . . . . 28

2.2.10 WB analysis for determination of protein expression levels . . 29

2.2.11 Quantification of SNARE ammounts in PNS and early endosomes from PC12 and in LS1 fraction from rat brain homogenates ......................... 29

2.2.12 Analysis of EEA1 membrane recruitment . . . . . . . . . . 29

2.2 .13 Immunocytoshemistry . . . . . . . . . . . . . . 30

2.2.14 Uptake and trafficking assays . . . . . . . . . . . . . 30

2.2 .15 Norepinephrine release . . . . . . . . . . . . . . . . 31

2.2.16 NGF-induced differentiation of PC12 cells . . . . . . . . . 31

2.2.17 Fluorescence Microscopy . . . . . . . . . . . . . . . . . . . . 32

$\begin{array}{lll}3 & \text { Results } & 33\end{array}$

3.1 Specificity in SNARE function . . . . . . . . . . . . . 33

3.1.1 Synaptobrevin, the most abundant endosomal SNARE, is not involved in early endosomal fusion . . . . . . . . . . . . . 33 
3.1.2 Cognate and non-cognate SNAREs occupy common microdomains on the endosomal membrane . . . . . . . . . 35

3.1.3 Mass-action governs SNARE interactions . . . . . . . . . . 38

3.1.4 Specificity of SNARE pairing is ensured by two co-operative mechanisms ...................... 43

3.2 Endosomal function upon SNARE knock-down . . . . . . . . . . . . . 48

3.2.1 Knock-down of early endosomal and exocytic SNAREs . . . . 50

3.2.2 Endosomal function is not affected in the knock-down cells . . 56

3.2.3 NGF-differentiation and exocytosis remain unaffected upon SNARE downregulation . . . . . . . . . . . . . 61

3.2.4 SNARE silencing does not affect early endosomal fusion in vitro 62

3.2.5 SNARE associations persist in the knock-down background, despite the low SNARE amounts . . . . . . . . . . 66

3.2.6 SNARE clustering on the endosomal membrane . . . . . . . . 69

3.2.7 The role of fusion-docking co-regulation . . . . . . . . . . . 72

4 Discussion 77

4.1 Regulation of specificity in SNARE-mediated fusion . . . . . . . . . . 78

4.1.1 SM proteins contribute to SNARE pairing specificity . . . . . 79

4.1.2 SM proteins control SNARE complex assembly . . . . . . . 79

4.1.3 Tethering factors determine membrane fusion sites and recruit the fusion machinery . . . . . . . . . . . . . . 82

4.2 The response of the fusion machinery to SNARE knock-down . . . . . 84

4.2.1 SNAREs are abundant and organize in membrane clusters . . 86

4.2.2 Co-operative functions of the docking and fusion machinery . 90

4.3 Conclusions . . . . . . . . . . . . . . . . . . . 92

$\begin{array}{lll}5 & \text { Summary } & 93\end{array}$

A Appendix

A.1 Monte Carlo simulation of SNARE interactions on the endosomal

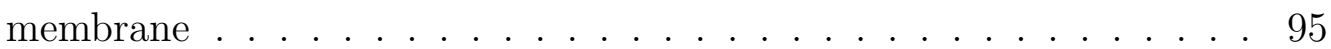

A.2 Calculation of the number of fused and docked organelles . . . . . . . 100 
Bibliography

Curriculum Vitæ

List of Publications 


\section{List OF Figures}

1.1 The essential steps of membrane traffic . . . . . . . . . . . . . . 3

1.2 The SNARE conformational cycle during vesicle docking and fusion. . 7

1.3 The endocytic pathway in mammalian cells . . . . . . . . . . . . . . . 12

1.4 Synaptobrevin interacts with the early endosomal SNAREs . . . . . . 15

1.5 Promiscuous SNARE interactions on early endosomes . . . . . . . . . 16

1.6 The non-cognate SNARE interactions represent genuine SNARE complexes in endosomal membranes . . . . . . . . . . . . . . . . . 17

3.1 Synaptobrevin is not involved in homotypic fusion of early endosomes 34

3.2 Formation of enlarged endosomes in PC12 cells transfected with GFP-

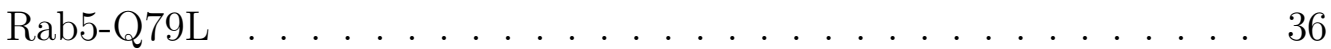

3.3 Intensity profiles along the membrane of an enlarged endosome costained for a pair of endosomal SNAREs . . . . . . . . . . . 37

3.4 The cognate and non-cognate SNAREs largely colocalize in microdomains on the endosomal membrane . . . . . . . . . . . . . 38

3.5 Mass action determines cis-complex formation . . . . . . . . . . . . . 42

3.6 Synaptobrevin appears to 'avoid' the interfaces between endosomes . 45

3.7 Fusion specificity is achieved by synergistically operating mechanisms 46

3.8 Transient knock-down of SNAREs in PC12 cells . . . . . . . . . . . . 51

3.9 The expression of individual SNAREs is efficiently downregulated in the knock-down cell lines . . . . . . . . . . . . . . . . . . . . . 54

3.10 The expression of other SNAREs does not change in the stably knocked-down cells . . . . . . . . . . . . . . . . . 55 
3.11 Transferrin, LDL and Dextran trafficking is not affected in knockeddown cells . . . . . . . . . . . . . . . . . . 5 58

3.12 Various endocytic pathways are not affected by transient knock-down of early endosomal SNAREs . . . . . . . . . . . . . . . . 59

3.13 Transferrin trafficking is not affected by the simultaneous knock-down of two SNAREs . . . . . . . . . . . . . . . . . . 6 60

3.14 Endosomal fusion functions in stably knocked-down cells . . . . . . . 61

3.15 NGF differentiation and norepinephrine release are not altered upon SNARE downregulation . . . . . . . . . . . . . . 63

3.16 Downregulation of individual SNAREs does not affect the fusion of early endosomes in vitro . . . . . . . . . . . . . . . . . . . 65

3.17 Changes in cognate SNARE associations upon downregulation of noncognate partners . . . . . . . . . . . . . . 6 67

3.18 Less than $10 \%$ of synaptobrevin binds to syntaxin 13 . . . . . . . 69

3.19 SNAREs are clustered on the endosomal membrane . . . . . . . . . 71

3.20 SNAREs are restricted from interactions due to clustering on the endosomal membrane . . . . . . . . . . . . . . . . 73

3.21 Enhanced recruitment of EEA1 on syntaxin 13 KD endosomes . . . . 74

3.22 More EEA1 is recruited on the membrane of knock-down organelles . 75

3.23 Docking is enhanced upon silencing of early endosomal SNAREs . . . 76 


\section{LisT OF TABLES}

3.1 Quantification of exocytic and early endosomal SNAREs in different preparations ............................ 40 


\begin{abstract}
Soluble N-ethylmaleimide-sensitive factor attachment receptor (SNARE) proteins catalyze organelle fusion in the secretory pathway. Different fusion steps are mediated by specific SNARE sets. By studying homotypic early endosomal fusion in neuroendocrine PC12 cells, I investigated how the specificity of SNARE function is regulated. Even though early endosomal and exocytic SNAREs promote distinct fusion events (early endosomal fusion and exocytosis, respectively), they colocalize on the early endosomal membrane and associate in promiscuous cis-complexes, in a mass-action-dependent manner. I showed that two combinatorial mechanisms account for the specificity in SNARE pairing and function: the enrichment of the necessary SNAREs at the prospective fusion sites, and preference for cognate SNARE associations in trans. To test the robustness of this highly regulated system under stress conditions, such as poor availability of its components, I downregulated by siRNA means the amount of single early endosomal or exocytic SNAREs in PC12 cells. Surprisingly, knock-down of early endosomal SNAREs, alone or in combinations, did not result in measurable changes of endosomal trafficking or fusion. I found that the residual SNARE levels (typically $\sim 10 \%$ ) were sufficient for a substantial amount of SNARE-SNARE interactions and that in wild type cells most SNARE molecules were concentrated in clusters, constituting a spare pool not readily available for interactions. Additionally, the organelles derived from the knock-down cell lines recruited more of the tethering factor EAA1 and exhibited enhanced docking. I therefore conclude that, surprisingly, SNAREs are expressed at much higher levels than needed for maintenance of organelle fusion, and that loss of SNAREs is compensated for by the co-regulation of the docking machinery, providing an additional example on the importance of cooperative functions for the regulation of intracellular fusion.
\end{abstract}




\section{Acknowledgments}

First of all, I would like to thank my supervisor, Prof. Reinhard Jahn - for his continuous trust and support, for his advice and guidance and for being an inspiring teacher.

My dearest thanks to Dr. Silvio Rizzoli. He guided me with amazing patience in my first scientific steps, he showed me that research can be a lot of fun and he always managed to transform my experimental disappointments to exciting new questions. Unlike his fruitless efforts to teach me how to use commas, he introduced me to the philosophy hidden in science. Silvio, thank you for being a mentor.

Many thanks to Prof. Nils Brose and Prof. Evgeni Ponimaskin, for being members of my PhD committee and for advising me in my work.

I want to thank all my colleagues at the Department of Neurobiology for making everyday work so easy and fun. I am grateful to Gottfried Mieskes for his constant help and to Michaela, Dagmar and Maria for assistance with cell culture and cloning. Many thanks to Ulf, for sharing our excitement and frustration on the endosomal issues, to Constanze, for - not only - musical discussions, to Raj, for the Indian enlightenment and to Nathan, for his advice and encouragement.

My special thanks to our 'corner gang', Alexander, Anand and Matias - I never admitted to them how much I enjoyed when they tried to make my life difficult! I thank them for spicing up every single day.

These years at the lab would not have been the same without Sina. Our endless conversations were a refugee for the bad moments and a good companion in everyday routine. My dear Suzannette, thank you for all the emotional support and the friendship.

I want to thank the IMPRS Neuroscience program and in particular, Michael 
Hörner and Sandra Drube for the organization and help.

Away from home, I made a new family here. My dear friends, thank you for always being there to listen and share, to laugh and party! Alexandra, Foteini, Phil, Michael, Alexandre, Felipe and Corinna - I am so happy to have met you!

My deepest thanks go to my family for their love, care and belief in me.

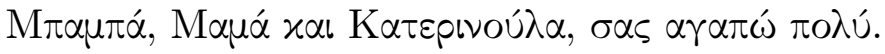

Finally, I want to thank Stephan - I would not have managed without your support. You showed me how to appreciate life, you always cured disappointment with smiles and you tolerated with love all my 'greek tragedies'. From Jim Knopf to Jack, I thank you for the journey.

'Seinen eigentlichen Anfang nahm das Abenteuer erst, als sie in Löwenberg ausstiegen.' I am looking forward to ours. 


\section{1}

\section{INTRODUCTION}

- An eukaryotic animal cell ... appears to operate a number of discontinuous circulatory systems ... [with] the receiving compartment [acting] as an efficient sink so that backflow or diffusion up the pathway is prevented. 9

Symposium on Membrane Recycling, Pitman Press, Bath, UK, 1982, G.E. Palade

Eukaryotic cells exhibit a highly compartmentalized organization, with a daedalian network of membranes, defining the cellular borders to the extracellular milieu as well as the numerous intracellular organelles. With the Golgi being the first organelle identified at the end of the 19th century, it was soon appreciated that the existence of membrane-enclosed compartments serves to spatially and temporally organize the various intracellular biochemical processes from each other, preventing undesired cross-reactions and providing high metabolic efficiency. Nevertheless, communication between the different compartments and the extracellular space is also vital for the exchange of proteins and lipids, the supply of nutrients and the disposal of toxic byproducts. More specifically, newly synthesized proteins are released extracellularly following the secretory pathway from the ER to the plasma membrane, while internalized material is targeted through the endocytic pathway to various interacellular organelles.

Exhange of material among the different compartments (especially the ones that constitute the endocytic and secretory network) is mediated by the intracellular trafficking of small, membrane-bound vesicles and tubulovesicular structures, a principle that was first described by Palade (summarized in Palade (1975)). A vesicle car- 
rying the appropriate cargo is generated from the donor compartment, pinching off its membrane (a process called budding), is transported, tethered to the target membrane, and finally fused to release its cargo (Figure 1.1).

Although the flow of cargo through the endocytic and secretory pathway is extensive, it does not compromise the identity of the constituent compartments. The mechanisms ensuring the structural and biochemical integrity of the different organelles lay in the principles of 'molecular sorting' and 'vesicle targeting'. First, membrane and soluble components are selectively incorporated into or excluded from the newly-formed transport vesicles. In this way, the material needed to leave the donor compartment is sorted for transport, without endangering the organelles' composition. Second, the nascent budded vesicles carry information that dictates their specific targeting and fusion with the appropriate acceptor compartment. A great variety of factors that mediate the regulated transport of cargo along the endocytic and secretory pathway has been characterized, including the Rab GTPases, tethering complexes, AAA-type ATPases, and SNAREs (Jahn and Scheller, 2006; Pfeffer, 2001). Interestingly, multiple homologues of these evolutionary-conserved molecules can be found in eukaryotic cells, complicating the issue of trafficking specificity, since the basic reactions of vesicular transport follow common mechanisms for any combination of compartments involved (with the exception of mitochondria and peroxisomes).

Therefore, the specific recognition between different compartments is presumably achieved by the unique combination of mutually matching factors which, once achieved, allows fusion of the interacting compartments. The sequential events of membrane recognition, interaction, tethering and fusion, and the co-operative function of different elements, seem to ensure the impeccable transport of cargo to the appropriate target compartment, although the detailed molecular mechanisms underlying these processes remain unknown.

\subsection{SNAREs: key players of membrane fusion}

The formulation of the SNARE hypothesis was the product of the novel experimental tools that were applied in the field of cell biology in the 1980s and 1990s. The 


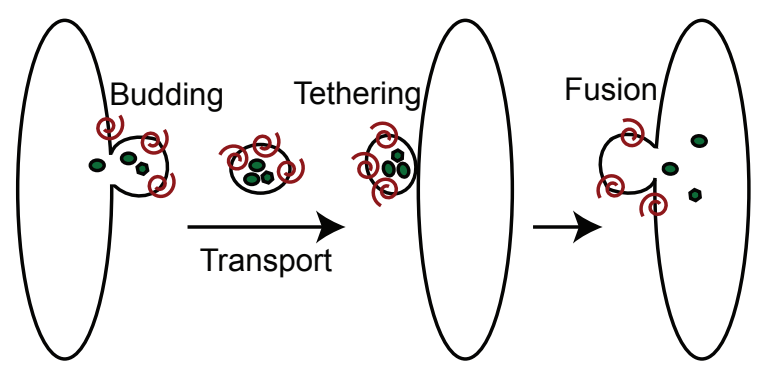

Figure 1.1: The essential steps of membrane traffic. First, a specific transport intermediate (vesicle), carrying the correct cargo (receptors, in red; soluble cargo, in green), is formed and buds from the donor organelle. The vesicle is subsequently transported to its target membrane by using the cell's microtubule or actin filament system. Vesicle and target membrane are then brought into close proximity by a process called tethering, which leads to the final fusion of this vesicle with the target organelle and the release of the cargo. Figure modified from Grosshans et al. (2006).

application of genetics and the reconstitution of membrane fusion in vitro provided the first evidence on the key role of SNARE proteins in intracellular fusion events. According to the model -which was later supported by a considerable amount of work- SNARE proteins that are localized in opposing membranes associate in a fourhelix bundle and drive membrane fusion by using the energy released by the complex formation. The formation of this bundle brings the membranes in closer apposition and promotes the membrane merger. The recycling of free SNARE molecules is achieved through the dissociation of the helical bundle, by the AAA+ protein NSF (N-ethylmaleimide-sensitive factor).

The in vitro reconstitution of the fusion between ER-derived or early Golgi vesicles and Golgi membranes (Fries and Rothman, 1980) provided the possibility to characterize the protein machinery required for vesicle targeting and fusion. As it was fast realized that the reaction was sensitive to the reagent NEM $(\mathrm{N}-$ ethylmaleimide), the ATPase NSF (NEM-sensitive-factor) was the first component of the fusion machinery to be isolated and therefore falsely believed to directly mediate the fusion process (Block et al., 1988). Few years later, three isoforms of NSF co-factors, named $\alpha^{-}, \beta$-, and $\gamma$-SNAPs (soluble NSF attachment proteins) were identified (Clary et al., 1990). In parallel, genetic screens in S. cerevisiae for the 
isolation of mutants with defects in the secretory pathway led to the identification of the yeast homologues for NSF and $\alpha$-SNAP, SEC18 and SEC17, respectively (Novick et al., 1980). Finally, using NSF and $\alpha$-SNAP as baits, a complex of three proteins was isolated from rat brain extracts (Söllner et al., 1993). Due to their affinity for $\alpha$-SNAP, the isolated proteins were named SNAREs (SNAP receptors) and their significance in neurotransmitter secretion was soon appreciated. The specific cleavage of each of these proteins by clostridial neurotoxins (tetanus and botulinum neurotoxins A, B, C1, D, E, F and G) (Jahn and Niemann, 1994; Montecucco and Schiavo, 1994) impaired neuronal exocytosis, providing the first functional link between SNAREs and membrane fusion. These proteins were no others than the now well-characterized neuronal exocytic SNAREs synaptobrevin, syntaxin 1 and SNAP25 and the work done on their characterization revealed biochemical and biophysical properties that are shared among all members of the SNARE family.

\subsubsection{SNARE structure}

SNAREs consist a superfamily of small proteins (around 100-300 amino acids in length), with 25 known members in yeast and around 36 distinct isoforms in mammalian cells (Bock et al., 2001). The characteristic, evolutionary-conserved sequence that defines all members of the family, the SNARE motif, consists of 60-70 residues with eight ampiphatic, heptad repeats, which are prone to form coil coiled structures (Hong, 2005). Most of the SNAREs (31 out of the 36 mammalian ones) are membrane anchored, via a hydrophobic transmembrane region present at their C-terminal, adjacent to the SNARE motif. SNAREs lacking a transmembrane domain, such as SNAP-25, SNAP-23 and Ykt6 possess hydrophobic post-translational modifications for membrane binding. Additionally, many SNARE molecules contain independently-folded domains at their N-terminus that are used as a criterion for a further classification of SNARE proteins.

\section{SNARE classification}

According to the SNARE hypothesis, SNAREs from opposing membranes can spontaneously assemble into a complex and thus promote membrane merging. Depending 
on their compartment of residence, SNAREs were initially classified as v-SNAREs (vesicle membrane SNAREs, when found on the 'donor' compartment) and as tSNAREs (target membrane SNAREs, when present on the 'acceptor' compartment) (Söllner et al., 1993). Since such a categorization could not be used in the case of homotypic fusion, an alternative terminology was adopted based on the structural characteristics of the proteins. The crystal structure of the neuronal (Sutton et al., 1998) and late endosomal (Antonin et al., 2002) SNARE complex revealed a high degree of conservation and showed that the complex consists of a twisted bundle of four helices, each one contributed by a SNARE motif. Additionally, 16 layers of interacting side chains were identified along the center of the complex and perpendicular to its axis, holding the four helices together. The residues participating in these interactions are hydrophobic with the exception of the ones occupying the central, so called '0'-layer, where three highly conserved glutamine (Q) residues and one highly conserved arginine $(\mathrm{R})$ residue can be found. Therefore, according to the aminoacid present at the '0' position of their SNARE motif, SNAREs were named as Q- or R-SNAREs. Additionally, since functional SNARE motifs proved to always be hetero-oligomeric (three Q-SNARE motifs and one R-SNARE), the position of the molecules in the helical bundle further led to the $\mathrm{Qa}, \mathrm{Qb}, \mathrm{Qc}$ and $\mathrm{R}$ classification (Bock et al., 2001; Fasshauer et al., 1998).

\section{SNARE N-terminal regions}

Extended N-terminal domains with coiled-coil structures can be found in most of the SNAREs. A three helical (Habc) bundle is characteristic of the Qa/syntaxin family. More specifically, in the case of syntaxin 1 and 7, the bundle can interact with the C-terminal SNARE motif, resulting in a so called closed conformation (Dietrich et al., 2003; Fasshauer, 2003). SNARE complex formation cannot occur under these conditions, and several regulatory molecules have been suggested to re-establish complex-competent, open conformation. The family of soluble SM (Sec1/Munc18related) proteins have been shown to be essential for fusion (Gerst, 2003) and to associate with Qa SNAREs, however in two different modes : either by stabilizing the closed conformation or by only associating with the N-terminal end of the SNARE. 
Another type of N-terminal extension is found in R-SNAREs like Ykt6 and Sec22b and it is known as longin domain (Dietrich et al., 2003). These are profilin-like domains and in case of Ykt6, can also associate with the C-terminal SNARE motif. Finally, the evolutionary younger family of R-SNAREs called brevins are devoid of a N-terminal motif and possess only few aminoacids after their SNARE motif (Jahn and Scheller, 2006). The necessity of a structured N-terminal SNARE motif for fusion regulation remains controversial.

\subsubsection{SNARE function and its regulation in membrane fusion}

\section{The zippering hypothesis and SNARE-mediated fusion}

SNAREs have been proposed to catalyze membrane fusion via their association in complex following a 'zippering' mode as described in Figure 1.2. This model requires the existence of at least one SNARE with a transmembrane domain on each of the membranes about to fuse. Assembly of the bundle starts at the N-terminus of the SNARE motifs and then proceeds in a zipper-like fashion towards the C-terminal, till a trans-SNARE complex is formed. The SNARE bundle is an extremely stable structure (it is resistant to $80^{\circ} \mathrm{C}, 8 \mathrm{M}$ urea or $2 \%$ SDS) (Fasshauer et al., 2002) and the mechanical force that is exerted on the membranes by its formation is believed to provide the necessary energy for lipid mixing and fusion (Jahn and Scheller, 2006). After fusion, the assembled SNARE complexes are found residing on the newly fused membrane, in a cis-configuration. Cis-SNARE complexes are fusion incompetent, and their disassembly is necessary for the recycling of free, reactive SNARE molecules. This process is mediated by the enzymatic activity of NSF, a hexameric member of the AAA+ protein family (Block et al., 1988). To perform its function, NSF requires binding to its co-factor SNAP (one of the three highly conserved isoforms, $\alpha-, \beta$-, or $\gamma$-SNAPs) which can directly bind to SNAREs.

\section{Regulation of SNARE function and sorting}

SNAREs may be considered the key catalysts of membrane fusion but they do not function alone. Multiple proteins have been shown to bind to free SNAREs or to pre-assembled SNARE complexes and specifically fine-tune their biophysical and 


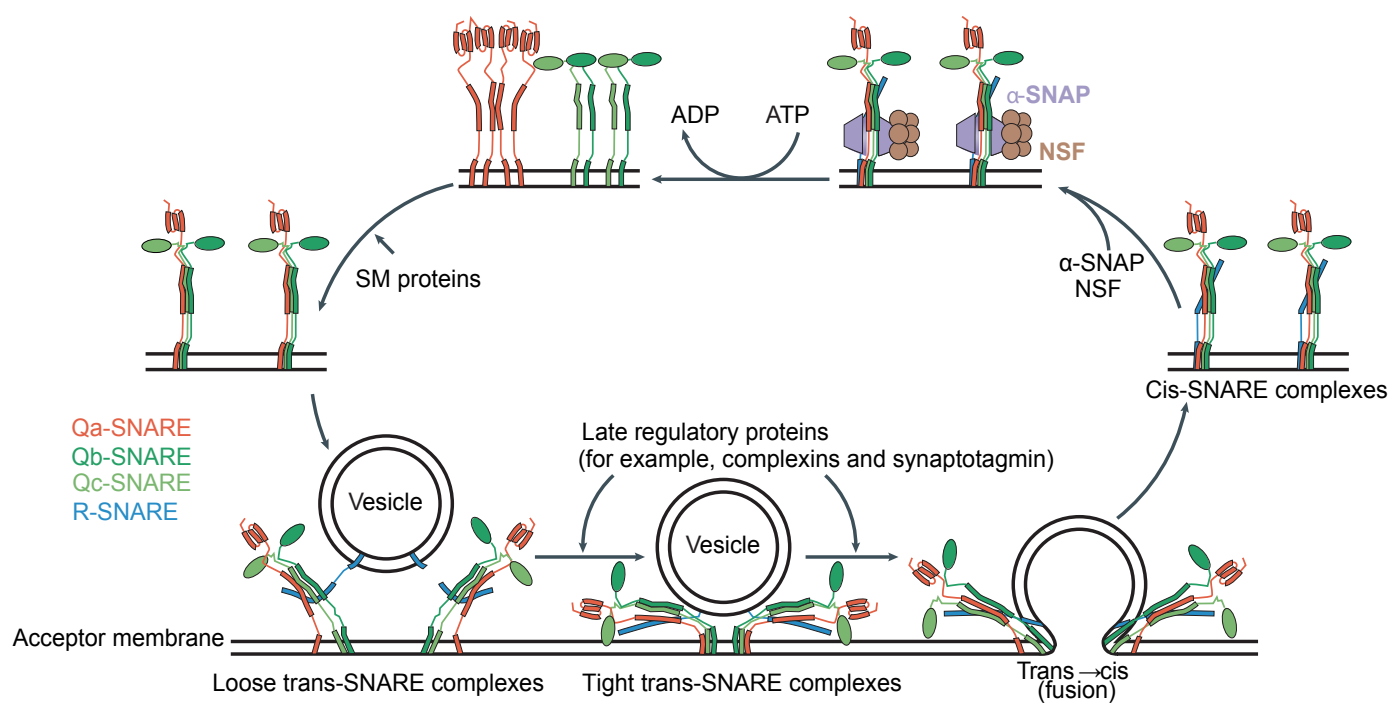

Figure 1.2: The SNARE conformational cycle during vesicle docking and fusion. As an example, three Q-SNAREs on an acceptor membrane and an R-SNARE on a vesicle are shown. QSNAREs, which are organized in clusters (top left), assemble into acceptor complexes, and this step might require SM proteins. Acceptor complexes interact with the vesicular R-SNAREs through the N-terminal end of the SNARE motifs, and this initiates the formation of a trans-complex. Transcomplexes proceed from a loose to a tight state (via a zippering procedure), and this is followed by the opening of the fusion pore. In regulated exocytosis, these steps are controlled by late regulatory proteins like complexins or synaptotagmins. During fusion, the strained trans-complex relaxes into a cis-configuration. Cis-complexes are disassembled by the AAA+ protein NSF together with its co-factors SNAPs. The R- and Q-SNAREs are then separated by sorting. Figure modified from Jahn and Scheller (2006).

functional properties. These include the correct sorting and targeting of SNAREs to the correct compartment, their competence for complex assembly, the stability and activity of the trans-SNARE complex.

As already discussed, the conformation of free SNARE molecules and their availability for complex formation is partly regulated by members of the SM family, with the exact role of these proteins remaining unclear. Studies with SNAREs of the secretory pathway indicate that SM proteins act positively to promote SNARE assembly perhaps by allowing for the transition of SNAREs from inactive-closed to active-open conformations. However, studies on the neuronal SNARE complex contradict each other attributing both negative and positive roles to Munc-18 (Gerst, 
2003; Wickner and Schekman, 2008).

Alternative regulators of trans-SNARE complexes are members of the synaptotagmin and complexin families. Synaptotagmin I is considered the $\mathrm{Ca}^{2+}$-sensor of fast synaptic vesicles exocytosis and interacts both with SNAREs and with acidic phospholipids in a $\mathrm{Ca}^{2+}$-dependent manner. Following $\mathrm{Ca}^{2+}$ binding, synaptotagmin is thought to promote fusion, although it is still unclear to what extent this is caused via its binding to SNAREs (Rizo et al., 2006). Another example of SNARE binding-protein that responds to $\mathrm{Ca}^{2+}$ elevation in neurosecretion is complexin; this molecule is believed to partially associate with SNARE complexes and unbind once the calcium triggers arrives (Tang et al., 2006). Finally, the macromolecular complex HOPS is believed to stabilize trans-SNARE complexes for the vacuole homotypic fusion in yeast (Wickner and Schekman, 2008).

Targeting of SNAREs to the compartment of their function is of high importance for the subsequent faultless function of the endocytic and secretory pathways and the interaction of SNAREs with coat proteins and tethering factors contributes to their correct sorting. The ER-Golgi SNAREs Bet1, Sed5 and Sec22 were shown to bind to the Sec23-Sec24 subcomplex of the coatomer complex, COPII, VAMP4 to interact with the adaptor protein, AP1, at the TGN (Jahn and Scheller, 2006) and recently, VAMP7 to bind to the clathrin adaptor ArfGAP Hrb (Pryor et al., 2008) and vti1b to epsinR (Miller et al., 2007). The Golgi tethering factor p115 was shown to promote the assembly of two syntaxin 5-containing SNARE complexes (Pfeffer, 2007). Last but not least, the early endosomal tether EEA1 possibly promotes the specificity of early endosomal SNARE pairing by interacting with the early endosomal SNAREs syntaxin 13 and 6 (McBride et al., 1999; Simonsen et al., 1999). However, due to the nature of SNAREs' intracellular role, it is expected that SNAREs not only localize at the compartments of their function but also in membranes of organelles that are part of the trafficking pathway they mediate. Some SNAREs, including members of the early endosomal set actually exhibit a very broad distribution, such as syntaxin 6 (Wendler and Tooze, 2001) and VAMP4 (Brandhorst et al., 2006; Steegmaier et al., 1999). Therefore, the localization of a SNARE alone cannot be informative on the fusion step this isoform mediates. 


\subsubsection{Specificity in SNARE function: a controversial issue}

The high conservation of the SNARE motif and the co-residence of several SNARE molecules on interacellular membranes irrespective of their compartement of function raise the question of regulation in fusion specificity. Without underestimating the role of docking/tethering factors in the recognition of compartments before fusion (Pfeffer, 2001), the contribution of SNARE identity in this regulatory step remains controversial. In in vitro assays, mammalian or yeast SNAREs that participate in distinct fusion events can associate promiscuously, with no preference observed towards cognate associations (Fasshauer et al., 1999; Tsui and Banfield, 2000; Yang et al., 1999). In accordance, in liposome fusion experiments with reconstituted mammalian SNAREs, associations between exocytic and endosomal SNAREs could promote fusion even though these two SNARE sets do not overlap functionally in vivo (Brandhorst et al., 2006). Additionally, more than one combination in the topology of early endosomal SNAREs on liposomes proved fusogenic (Zwilling et al., 2007). However, fusion of yeast SNARE-containing liposomes gave indications of both topological (Parlati et al., 2000) and pairing (McNew et al., 2000; Paumet et al., 2004) specificity, even though promiscuity in SNARE associations was not excluded as any R-SNARE could promote fusion with plasma-membrane Q-SNAREs (McNew et al., 2000). In exocytosis of PC12 cells, specificity in SNARE pairing was observed, as cognate SNAREs were more successful than the non-cognate ones in blocking release in a cell-free assay (Scales et al., 2000).

It is not debatable that several SNAREs operate in distinct fusion events involving different processes and organelles (Jahn and Scheller, 2006). Indeed, deletion of a given SNARE or downregulation by siRNA means results in specific impairment of intracellular trafficking. For example, exocytosis was blocked in neurons when synaptobrevin (Schoch et al., 2001) or SNAP-25 (Washbourne et al., 2002) were knocked out, and deletion of Ykt6p inhibited ER-to-Golgi transport in yeast (McNew et al., 1997). Knock-down of syntaxin 10 led to missorting of mannose-phosphatereceptors (Ganley et al., 2008) and downregulation of syntaxin 16 and 5 affected endosome to Golgi trafficking (Amessou et al., 2007). Nevertheless, in multiple cases, SNARE knock-out or knock-down had little impact on cell function, with 
no measurable changes being observable in the intracellular pathways in which the targeted SNAREs are known to play a role. knock-out of the late endosomal SNARE vti1b had neither an effect on the animals' fertility or viability, nor on late endosomal and lysosomal function (Atlashkin et al., 2003), while syntaxin 1 deficient mice were fertile and viable (Fujiwara et al., 2006). SNAP-25 and SNAP-23, as well as VAMP2/synaptobrevin-2 and cellubrevin, can substitute for each other to a varying degree in the norepinephrine exocytosis of chromaffin cells (Borisovska et al., 2005; Delgado-Martnez et al., 2007) and similarly in yeast, the loss of Sec22 and Pep12p is partially rescued by Ykt6 and Vam3p, respectively (Darsow et al., 1997; Liu and Barlowe, 2002).

Therefore, important aspects on the regulation of specificity in SNARE pairing and function remain obscure and the mechanisms that ensure the high selectivity among cognate partners for fusion are still unclear. Nevertheless, considering the diversity of factors that function upstream of SNAREs in tethering and docking, one can expect that the high organization of the intracellular network of membranes is the product of the combinatorial action of multiple mechanisms that precede the final act of fusion.

\subsection{Early endosomes and endosomal trafficking}

- Endosomes are a heterogeneous population of endocytic vacuoles through which molecules internalized during pinocytosis pass en route to lysosomes. In addition to this transport function, recent studies indicate that these organelles also act as clearing houses for incoming ligands, fluid components and receptors.

Helenius A. et al., TIBS, 1983

\subsubsection{Endocytosis and early endosomes}

Eukaryotic cells are efficiently separated from the extracellular environment by the dynamic structure of the plasma membrane, which regulates the transport of 
molecules from and into the cytoplasm. While small molecules, like ions, sugars and aminoacids can easily transverse the plasma membrane by use of integral membrane proteins (ion channels or transporters), the entry of macromolecules is mediated by the formation of membrane-bound vesicles that include the cargo, invaginate towards the cytoplasm and pinch-off (bud from) the plasma membrane. This process is characterized by the general term of endocytosis and is essential for the maintenance of cellular homeostasis, uptake of nutrients, intercellular communication, neurotransmission, development and immune response. Indicative of its importance is the fact that many infectious components have developed mechanisms that incorporate them to the endocytic process and this way mediate their entry to the host cell.

There are several mechanisms for the internalization of material from the plasma membrane: phagocytosis, which is mainly used by specialized cells of the immune system, and pinocytosis, which includes macropinocytosis, clathrin-mediated endocytosis (CME), caveolae-mediated endocytosis, and clathrin- and caveolaeindependent endocytosis (Conner and Schmid, 2003). The most well characterized process is the receptor-mediated endocytosis that is responsible for the uptake of essential nutrients, such as the low-density lipoprotein (LDL) particles that bind to the LDL receptor, and the iron-laden transferrin (Tfn) that binds to the Tfn receptor (Mellman, 1996). This endocytic process involves the incorporation of receptor-ligand complexes into clathrin-coated pits, which subsequently pinch off the membrane to form clathrin-coated vesicles (Mukherjee et al., 1997). The first compartment that recently endocytosed vesicles meet and fuse with is the early/sorting endosome. Early endosomes are located at the periphery of the cell, exhibit a tubulovesicular morphology and serve as the main sorting station of the endocytic pathway where the intracellular destination of internalized cargo is decided. Due to the slightly acidic pH (around 6-6.8) of the early endosomal lumen, dissociation of many ligands from their receptors occurs in this compartment. The free receptors are then returned, directly or indirectly (through recycling endosomes), back to the plasma membrane, while the released ligands are targeted to degradation through sequential transport to late endosomes and lysosomes. In addition, early endosomes 


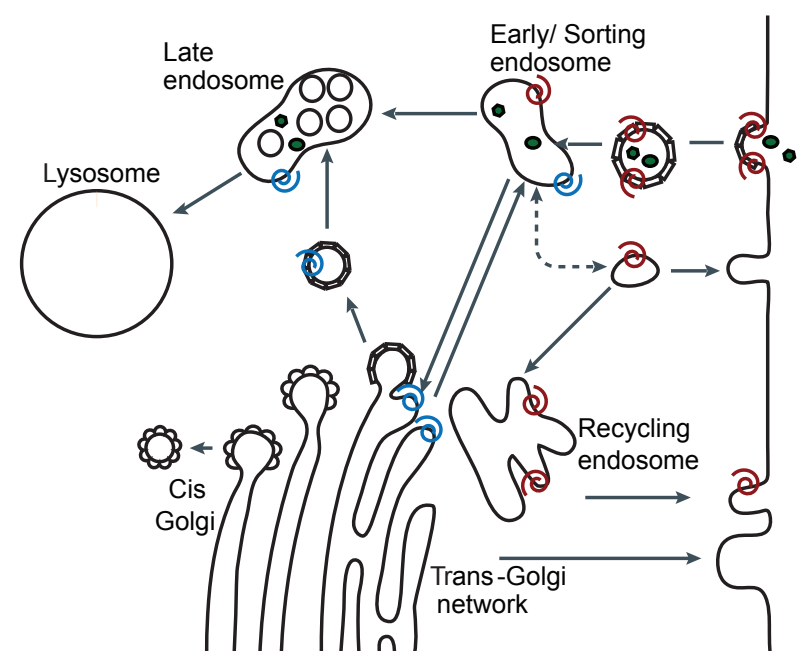

Figure 1.3: The endocytic pathway in mammalian cells. Arrows indicate the different intracellular routes. Examples of the transferrin receptor (in red) and of mannose-6-phosphate receptor (in blue) are schematically presented; soluble material in green. Figure modified from Jahn and Scheller (2006).

receive and send material to the trans-Golgi network (Cook et al., 2004; Itin et al., 1997), justifying their name as the major sorting organelles (Figure 1.3). Even though the early endosomal compartment is considered the first interacellular station mainly for cargo that follows clathrin-mediated endocytosis, there is increasing evidence that alternative internalization pathways may merge to the early endosome. More specifically, the bacterial Shiga and Cholera toxins, which achieve their entry to the host cell by binding to plasma membrane sphingolipids and gangliosides respectively, are believed to use the early endosomal compartment en route to Golgi and ER, independent of their mode of entry (which can depend or not on caveolin or clathrin; Lencer and Tsai (2003)). Finally, the early endosomal intermediate has also been implicated in the recycling of synaptic vesicles in neurons. One of the models of synaptic recycling suggests that the components of synaptic vesicles recycle from the membrane via clathrin coated carriers, which upon uncoating fuse with an endosomal structure. New synaptic vesicles are then believed to form from this endosomal intermediate (Sudhof, 2004).

Considering that mammalian cells internalize every hour ammounts of membrane that correspond to their total surface area (Steinman et al., 1976), it is not surpris- 
ing that the early endosome is a highly dynamic compartment. In addition, it does not only exhibit the ability of receiving internalized vesicles but also performing homotypic fusion (Gruenberg and Howell, 1989). Therefore, its steady-state is very variable and it is determined by the parallel processes of the fusion of incoming vesicles, the fusion of endosomes with each other and the budding of carrier vesicles to various destinations. Furthermore, according to a second model, early endosomes are not considered long-lived defined organelles but are rather believed to mature to late endosomes and lysosomes, in a process that involves gradual and specific changes in their molecular composition (Maxfield and McGraw, 2004). Even though the borders for defining a structure as part of the endosomal compartment seem blurry considering the multipotency of these organelles, many of the molecular players involved in the functional communication of early endosomes with other compartments and mainly in their homotypic fusion have been identified and can be used as molecular tags for structures that perform early in the endocytic pathway and exhibit a sorting role.

\subsubsection{Characteristics of homotypic early endosomal fusion: the SNAREs involved}

Early endosomal homotypic fusion follows the requirements of any other intracellular fusion process: it is time, temperature, cytosol and ATP dependent, and exhibits sensitivity to NEM, indicative of SNARE involvement in the process (Diaz et al., 1988). An essential factor of endosomal fusion that was identified early (Gorvel et al., 1991) is the small GTPase Rab5, by now the most well-characterized molecular marker of the early endosomal compartment. Rab5 remains in the cytosol when it is associated with GDP. However, once it binds to GTP, it is attached to the endosomal membrane and is believed to organize tethering/docking domains on the membrane by the recruitment of its effectors. Interestingly, many of the effector molecules have a positive feedback loop on the membrane stabilization of Rab5, with rabex-5 and rabaptin-5 preventing GTP hydrolysis and Vps34 producing more $\mathrm{PI}(3) \mathrm{P}$, which is the binding site of most Rab-5 effectors molecules (Zerial and McBride, 2001). In parallel, other Rab5 effectors, such as rabenosyn-5 and EEA1 associate with the 
members of the fusion machinery, possibly serving as links between the sequential processes of docking and fusion (McBride et al., 1999; Simonsen et al., 1999).

While the composition of the tethering/docking machinery that functions upstream of the early endosomal homotypic fusion is well defined, the characterization of the SNARE molecules working in this process has been the objective of multiple studies, with a variety of candidates proposed. The first molecules that were suggested by functional studies to play a role in early endosomal fusion were the Q-SNAREs syntaxin 13 and syntaxin 6 (Mills et al., 2001; Prekeris et al., 1998), which interestingly, were also found to interact with the tethering factor EEA1, as described above. Co-immunoprecipitation experiments further identified the interaction of syntaxin 6 and VAMP4 (Steegmaier et al., 1999) as well as the associations between syntaxins 6, 16, vtila and VAMP4 (Kreykenbohm et al., 2002). Antibodies against endobrevin seem to inhibit the endosomal fusion reaction (Antonin et al., 2000), while even the neuronal exocytic SNAREs, synaptobrevin and SNAP-25 have been implicated in the process (Aikawa et al., 2006; Sun et al., 2003).

A common complication in the identification of endosomal SNAREs raises from the fact that co-immunoprecipitation and localization data were used to functionally implicate several SNARE molecules in this fusion step. Even though each SNARE is expected to accumulate at the compartment in which exhibits its function, vesicular transport will inevitably spread the SNARE molecules in several compartments, making it hard to determine the fusion specificity of a SNARE solely by its distribution. Due to their central role in the endocytic pathway, early endosomes accomodate on their membrane a plethora of SNARE molecules that are passengers en route to their resident compartment. It is therefore not surprising that exocytic (SNAP-25, syntaxin 1 and synaptobrevin) and late endosomal SNAREs (syntaxins 7 and 8, vti1b, endobrevin) seem to localize at the endosomal compartment, as shown by Brandhorst et al. (2006). However, functional characterization of homotypic fusion in the same study by introduction of recombinant SNARE fragments, which compete with the function of the endogenous proteins, suggested that syntaxin 13 , 6, vtila and VAMP4 are the key mediators of homotypic fusion between early endosomes. Conversely, specific cleavage of SNAP-25 by BoNT/E in this study and 

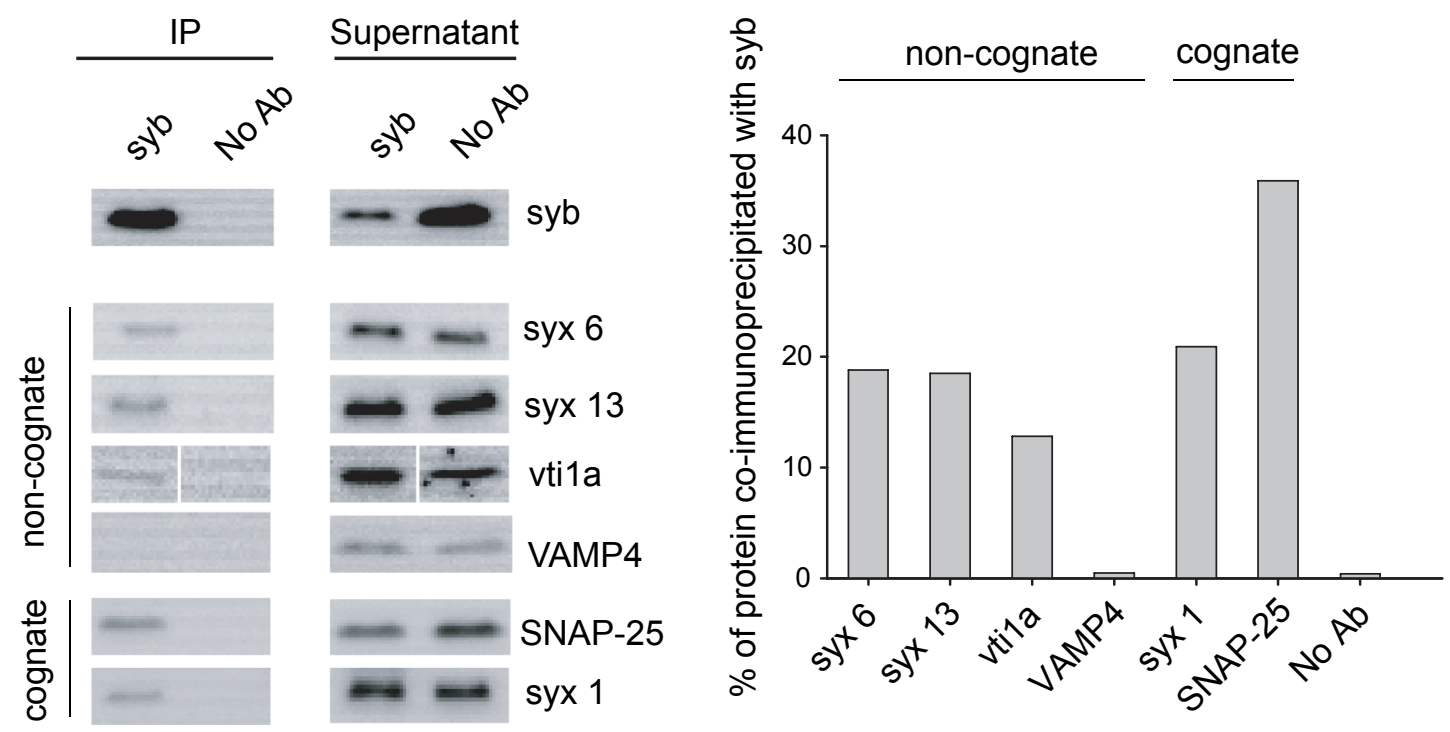

Figure 1.4: Synaptobrevin interacts with the early endosomal SNAREs. PNS from PC12 cells was centrifuged in a sucrose density gradient, a band highly enriched in early endosomes was isolated, and after solubilization with Triton X-100 immunoprecipitation was performed with a monoclonal antibody against synaptobrevin (left lane); controls are shown in the second lane (no antibody added). The corresponding supernatants are shown in the respective position (lanes 3-4). The interacting SNAREs were identified by immunoblot analysis. A total of $10 \%$ of the total sample is loaded in every lane. Typical blots of three independent experiments are shown. Graph: Quantification by densitometry of co-precipitation by densitometry, from the blots presented; the band intensities were normalized to starting material. The 'no antibody' value corresponds to the mean value of all negative controls presented.

of syntaxin 1 by BoNT/C1 (Rizzoli et al., 2006) did not affect endosomal fusion, excluding the participation of these proteins in this process, despite their presence on the endosomal membrane.

\subsubsection{Promiscuous SNARE associations on the endosomal membrane}

Therefore, the questions of specificity in SNARE pairing and function, that were discussed at section 1.1.3 seem to be particularly relevant for the endosomal compartment. How can the cognate SNAREs associate selectively for fusion on a membrane containing a variety of non-cognate SNAREs, with the intrinsic abilities of promiscuous interactions? Are there regulatory mechanisms that prevent the associ- 


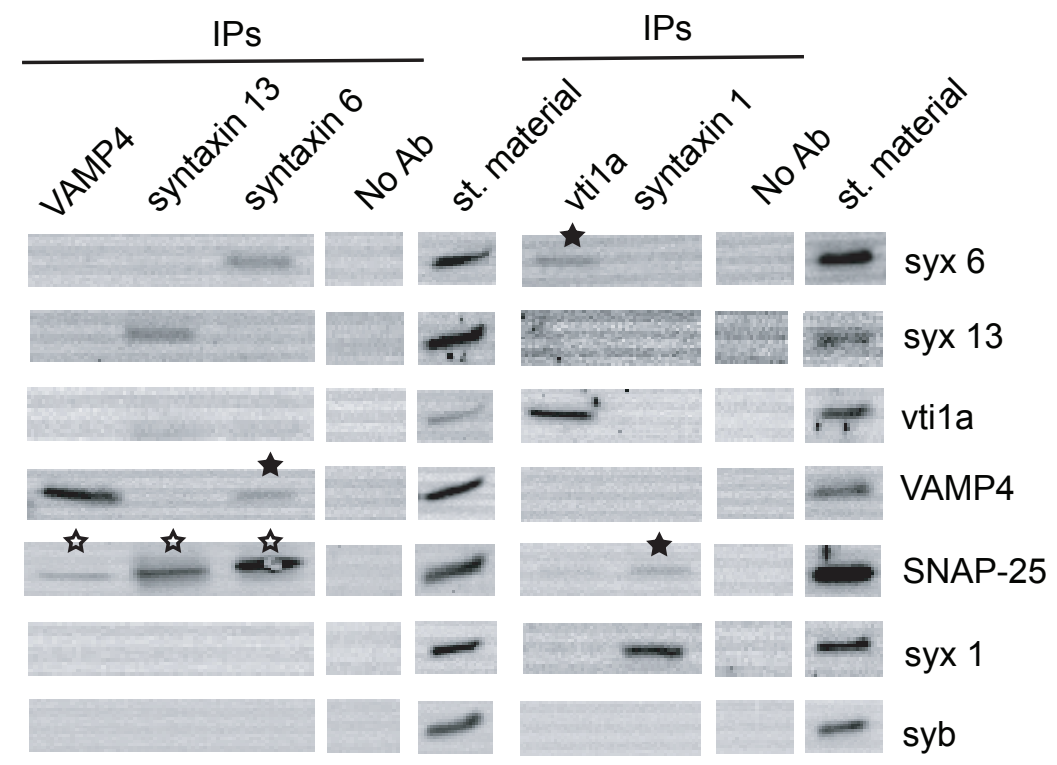

Figure 1.5: Promiscuous SNARE interactions on early endosomes. Immunoprecipitations were performed with antibodies against the SNAREs indicated at the top of the figure. All immunoprecipitates were analyzed by immunoblotting for the SNAREs indicated. The 'No Ab' value corresponds to the negative control, in which no antibody was added for immunoprecipitation. A total of $10 \%$ of the total sample is loaded in every lane. Note that SNAP-25 was omitted from the IP analysis because none of the available antibodies detects SNAP-25 in assembled SNARE complexes. Open stars indicate interactions between non-cognate SNAREs; filled stars indicate cognate associations.

ations among non-cognate SNAREs and ensure the functional separation of different SNARE sets? Or is the 'crosstalking' among non-cognate isoforms possible on the endosomal membrane, as observed in vitro, despite the functional redundancy of such associations? During my Master's thesis, I addressed the question of promiscuity in SNARE associations at the endosomal compartment. I purified early endosomes from PC12 cells by discontinuous gradient centrifugation and after solubilization, I performed immunoprecipitation using an antibody against synaptobrevin. Interestingly, the precipitants contained not only the exocytic SNAREs SNAP-25 and syntaxin 1 as expected, but also the three endosomal Q-SNAREs, syntaxin 13, 6 and vti1a, with no preference for the cognate SNAREs (Figure 1.4). The fact that the endosomal SNARE VAMP4 was not co-immunoprecipitated indicates that the 

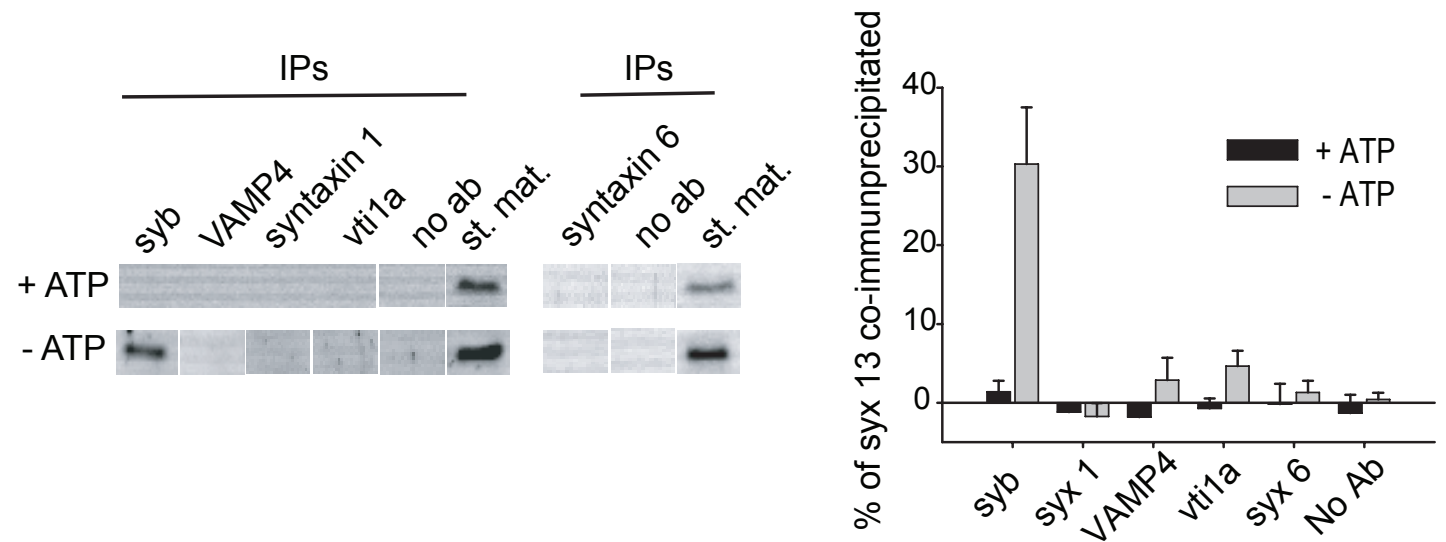

Figure 1.6: The non-cognate SNARE interactions represent genuine SNARE complexes in endosomal membranes. PNSs were incubated with rat brain cytosol in presence or absence of ATP and immunoprecipitations were performed after detergent extraction. Typical immunoblots for syntaxin 13 are shown. Note the presence of bands in the synaptobrevin precipitates in absence, but not in presence of ATP. Upon ATP depletion, NSF function is blocked and SNARE complexes accumulate, facilitating their detection. Quantification of syntaxin 13 co-immunoprecipitation is presented. Bands were quantified by densitometry (see Materials and Methods); averages \pm s.e.m. from three independent experiments are shown.

observed associations are part of genuine SNARE complexes that follow the QabcR rule. I subsequently performed immunoprecipitation against VAMP4, syntaxin 13, syntaxin 6 , vtila and syntaxin 1 and analyzed the precipitates for the presence of the other exocytic and endosomal SNAREs. A high degree of promiscuity was observed with SNAP-25 co-immunoprecipitating with VAMP4, syntaxin 13 and syntaxin 6, in parallel to the cognate associations between syntaxin 6 and VAMP4, vtila and syntaxin 6, and syntaxin 1 and SNAP-25 (Figure 1.5). To further substantiate the identity and properties of the observed interactions, I checked their sensitivity to NSF inhibition. Immunoprecipitation experiments were performed in presence or absence of ATP; since NSF is an ATPase, depletion of cytosol from ATP should block its activity. Focusing on syntaxin 13, no interactions were detected upon incubation in presence of ATP. However, upon ATP depletion, cognate and non-cognate interactions were observed, with the most dominant the association of syntaxin 13 with synaptobrevin (Figure 1.6). Interestingly, similar observations were made when 
a synaptosome-enriched rat brain fraction or intact PC12 cells were used as starting material. It can be therefore concluded that the promiscuous formation of genuine cis-SNARE complexes between exocytic and endosomal SNAREs is possible on the endosomal membrane and that the immunoprecipitation data cannot always be informative on the functional relation between interacting SNAREs.

\subsection{Aims of this study}

Considering the high degree of promiscuity in SNARE associations described above, the regulation of specificity in SNARE pairing is of vital importance for the impeccable function of the intracellular machinery. Nevertheless, some SNARE molecules seem to participate in more than one fusion events and in particular for the early endosomal compartment, a high variety of non-cognate SNAREs co-reside on the same membrane.

The goal of this thesis is to characterize the properties of the endosomal fusion machinery, to investigate the mechanisms that ensure the specificity on SNARE pairing and function at the endosomal compartment and understand the importance of early endosomal SNAREs in intracellular trafficking. To dissect the mechanisms that govern endosomal SNARE function, new experimental tools were established and a variety of experimental approaches was applied.

Using the early endosomes from PC12 cells as an experimental platform, I applied biochemical, microscopical and computational tools and studied the status of SNARE associations both in cis- and trans-configuration.

In an attempt to evaluate the function of endosomal SNAREs in vivo, I took advantage of the advancing field of RNAi silencing and downregulated the expression of SNAREs that reside on the endosomal compartment in PC12 cells. To this end, I designed and performed the creation of PC12 cell lines stably knocked down for individual SNAREs. In parallel, I developed a variety of cell-based assays to allow the investigation of intracellular trafficking under conditions of SNARE downregulation. Finally, I studied the status of SNARE interactions and the response of the fusion and docking machinery to SNARE downregulation. 


\section{Materials AND Methods}

\subsection{Materials}

\subsubsection{Chemicals}

All chemicals were purchased from Biorad, Boehringer, Merck, Roth, Serva and Sigma. Fluorescently-labeled markers were purchased from Molecular Probes/Invitrogen.

\subsubsection{Enzymes, kits and bacterial strains}

For the preparation of the shRNA-vectors used for the creation of the stably knockeddown cell lines, the kit accompanying the psiSTRIKE ${ }^{T M}$ Neomycin vector (Promega, Madisson, USA) was used. For the purfication of digestion reactions and proliferation of DNA plasmids, kits from Qiagen and Machery \& Nagel were used. Restristion enzymes were purchased from New England Biolabs. For cloning, the E.coli XL1-Blue strain from Stratagene was used.

\subsection{3 siRNA and shRNA sequences}

The siRNA oligos were designed using the BLOCK-iT ${ }^{T M}$ RNAi Designer (Invitrogen) and were purchased from the same company. The sequences used are: Syntaxin 6 (bp 375-400 and 385-410 of GenBank accession no. U56815), syntaxin 13 (bp 736-761 of GenBank accession no. AF035632), syntaxin 16 (bp 298-323 and 571-596 of GenBank accession no. NM_ 001108610), VAMP4 (bp 357-382 and 377-402 of GenBank accession no. AF061516), vti1a (bp 160-185 and 461-486 of GenBank accession no. NM_ 023101), vti1b (bp 664-689 and 677-802 of GenBank accession no. 
NM_ 016800), synaptobrevin (bp 395-420 of GenBank accession no. NM_ 012663).

Target sites for silencing the different SNAREs were selected and DNA oligonucleotide sequences were designed accordingly by using the siRNA Target Designer (Promega, Madisson, USA). The DNA sequences (MWG, Ebersberg, Germany) contained the chosen target site, a loop sequence, the reverse complement of the target site and the appropriate 5' and 3' ends for cloning into the psiSTRIKE U6 Hairpin Neomycin vector (Accession No. AY497508). The resulting plasmids were sequenced to ensure correct insertion of the shRNA sequences. The sequences used were:

vti1a (bp 684-702 of NM_023101):

5 '-ACCGTCTGCTCTCCCTTGATAATTCAAGAGATTATCAAGGGAGAGCAGACTTTTTC -3’

syntaxin 13 (bp 764-782 of AF035632):

5 '-ACCGTCCTCTCAGTGATTGTTATTCAAGAGATAACAATCACTGAGAGGACTTTTTC -3'

syntaxin 6 (bp 792-810 of U56815):

5 '-ACCGTCATGCTGGATGATTTCTTTCAAGAGAAGAAATCATCCAGCATGACTTTTTC -3'

synaptobrevin (bp 1298-1316 of NM_012663):

5 '-ACCGGAGTTCAGTGTGTTATGATTCAAGAGATCATAACACACTGAACTCCTTTTTC -3'

control sequence (scrambled sequence of synaptobrevin bp 966-984, NM_012663):

5' -ACCGCGTGGATACAGATTACCTTTCAAGAGAAGGTAATCTGTATCCACGCTTTTTC -3'.

\subsubsection{Buffers}

PBS (Phosphate Saline Buffer):150 mM NaCl/20 mM Naㄹ $\mathrm{HPO}_{4}, \mathrm{pH}$ 7.4.

Homegenization Buffer: $250 \mathrm{mM}$ sucrose, $3 \mathrm{mM}$ imidazole- $\mathrm{HCl}, \mathrm{pH}$ 7.4.

Extraction Buffer: PBS pH 7.4, 1\% Triton X-100, 5 mM EGTA, 5 mM EDTA.

Sample Buffer $(2 \times)$ : 100 mM Tris, $8 \%$ SDS, $24 \%$ glycerol, 0,02\% Serva Blue G, $4 \%$ $\beta$-mercaptoethanol, $\mathrm{pH} 6.8$.

Krebs solution: $130 \mathrm{mM} \mathrm{NaCl}, 4 \mathrm{mM} \mathrm{KCl}, 5 \mathrm{mM} \mathrm{CaCl}_{2}, 1 \mathrm{mM} \mathrm{MgCl} 2,48 \mathrm{mM}$ glucose, 10 mM HEPES-NaOH pH 7.3. 


\subsubsection{Antibodies}

The following antibodies were used for immunoprecipitation: rabbit polyclonal sera against syntaxin 13 and VAMP4 (Brandhorst et al., 2006); monoclonal antibodies against synaptobrevin ( $\mathrm{Cl}$ 69.1), available from Synaptic Systems, Göttingen, Germany. The following antibodies were used for Western Blot detection: monoclonal antibodies against Rab5 (Cl 621.1, (von Mollard et al. (1994), actin (Affinity BioReagents, Golden, CO), tubulin (Developmental Studies Hybridoma Bank), vtila, vti1b, EEA1 (BD Biosciences, Erembodegem, Belgium), VAMP3 and VAMP8 (Abcam, Cambridge, UK), synaptobrevin (69.1), syntaxin 13 (151.1), syntaxin 1 (78.2) (all Synaptic Systems); polyclonal sera against syntaxins 6, 16, VAMP4 (Bethani et al. (2007)), syntaxin 7 and 8 (Takamori et al. (2006)), syntaxin 1 (Lang et al. (2001)), SNAP-25 (Aguado et al. (1996)). HRP-conjugated goat anti-mouse and anti-rabbit secondary antibodies were purchased from BioRad (Hercules, CA). For immunostaining: rabbit polyclonal sera against syntaxin 6 , syntaxin 16 , syntaxin 13 , SNAP-25 (Bethani et al. (2007)), VAMP4 (Abcam, Cambridge, UK), monoclonal antibodies against vti1a, vti1b, EEA1 (BD Biosciences, Erembodegem, Belgium), syntaxin 6 (BD Biosciences, Erembodegem, Belgium), syntaxin 13 (151.1), synaptobrevin (69.1), syntaxin 1 (78.3), SNAP-25 (71.1) (Synaptic Systems, Göttingen, Germany). Goat anti-mouse and anti-rabbit fluorescently-labelled antibodies were purchased from Jackson Immunoresearch Europe (Newmarket, England). The following antibodies were added to the fusion reactions: rabbit polyclonal sera against syntaxins 1, 6, 13, 16, vti1a, synaptobrevin, VAMP4 (as described above), syntaxins 4 and 7 (Riedel et al. (2002)).

\subsection{Methods}

\subsubsection{Cell culture}

Neuroendocrine PC12 (pheochromocytoma) cells were grown in Dulbecco's modified Eagle's medium (DMEM) with the following additions: 5\% fetal calf serum (FCS), 10\% horse serum, $4 \mathrm{mM}$ glutamine and 100 units $/ \mathrm{ml}$ each of penicillin and streptomycin. Cells were grown at $37^{\circ} \mathrm{C}$ in $90 \% \mathrm{CO}_{2}$ and were passaged when 
reached confluency. Cells were used until their 20th passage. Cells, stably transfected with psiSTRIKE-shRNA neomycin vector, were grown in complete media containing $500 \mu \mathrm{g} / \mathrm{ml} \mathrm{G418} \mathrm{(PAA,} \mathrm{Pasching,} \mathrm{Germany).} \mathrm{The} \mathrm{minimum} \mathrm{amount} \mathrm{of}$ G418 necessary to eliminate non-transfected cells was determined to $500 \mu \mathrm{g} / \mathrm{ml}$ by growing PC12 under various concentrations of G418 (100-1000 $\mu \mathrm{g} / \mathrm{ml})$ for 10 days and counting the surviving cells every two days.

\subsubsection{Transfection of DNA plasmids and siRNA oligonucleotides}

\section{Electroporation}

Cells were grown to confluency in a $75 \mathrm{~cm}^{2}$ flask and harvested with Trypsin-EDTA (200 mg/L EDTA-500 mg/L Trypsin, Cambrex, East Rutherford, NJ). Cell pellet was resuspended in $2 \mathrm{ml}$ Cytomix, pH $7.6\left(120 \mathrm{mM} \mathrm{KCl}, 10 \mathrm{mM} \mathrm{K}_{2} \mathrm{HPO}_{4} * 3 \mathrm{H}_{2} \mathrm{O}\right.$, $10 \mathrm{mM} \mathrm{KH}_{2} \mathrm{HPO}_{4}, 0.15 \mathrm{mM} \mathrm{CaCl}_{2} * 2 \mathrm{H}_{2} \mathrm{O}, 2 \mathrm{mM}$ EGTA, $5 \mathrm{mM} \mathrm{MgCl}{ }_{2} * 6 \mathrm{H}_{2} \mathrm{O}, 25 \mathrm{mM}$ HEPES, $2 \mathrm{mM}$ ATP, $5 \mathrm{mM}$ GSH). $400 \mu \mathrm{l}$ of the suspension were added in a Gene Pulser Cuvette (Gene Pulser II, 0.2 cm electrode gap, BioRad), which already contained $20 \mu \mathrm{g}$ DNA. Cells were pulsed with the following settings: $1.15 \mathrm{kV}, 50 \mathrm{Ohm}$, $50 \mu \mathrm{F}$ and then plated in fresh medium.

\section{Lipofection}

PC12 cells were plated on poly-L-lysine coverslips the day before transfection in medium depleted from antibiotics. Before transfection of siRNA oligonucleotides, medium was changed to DMEM containing only $4 \mathrm{mM}$ glutamine. For transfection of cells contained in 1 well of a 24-well plate, $6 \mu$ siRNA (stock $20 \mu \mathrm{M}$ ) were diluted in $18 \mu \mathrm{l}$ OptiMEM and $6 \mu \mathrm{l}$ Lipofectamine 2000 (Invitrogen, Carlsbad, CA) in 50 $\mu \mathrm{l}$ OptiMEM. After 5 min incubation, the two solutions were mixed. 25 min later, the mix was brought to $100 \mu \mathrm{l}$ with addition of OptiMEM and was added to the well. Complete medium was replaced after 4 hours. Cells were studied $72 \mathrm{~h}$ posttransfection. For the trasfection of the GFP-Rab5-Q79L plasmid , $1 \mu \mathrm{g}$ of DNA was used per well (in a 24-well plate) and the transfection was performed according to manufacturer's instructions, using Lipofectamine 2000. Cells were processed $48 \mathrm{~h}$ post-transfection. 


\subsubsection{Preparation of postnuclear supernatant from PC12 cells}

Cells were grown in $150 \mathrm{~mm}$ culture dishes. After they reached confluency, the culture medium was removed from each plate and the plates were washed with $5 \mathrm{ml}$ saline PBS per dish. The cells were then harvested by trypsin/EDTA treatment (2 ml per plate; $200 \mathrm{mg} / \mathrm{L}$ EDTA-500 mg/L Trypsin, Cambrex, East Rutherford, $\mathrm{NJ}$ ). The reaction was terminated by addition of $5 \mathrm{ml}$ of cold culture medium; the de-attached cells were collected and centrifuged at 1,000 rpm (Varifuge 3.0R, Heraeus SEPATECH), for $5 \mathrm{~min}$, at $4^{\circ} \mathrm{C}$. The cells were resuspended in PBS, the centrifugation step was repeated and the resulting pellet was washed with ice-cold internalization medium (OptiMEM, Invitrogen, Carlsbad, USA, supplemented with $10 \mathrm{mM}$ glucose). After centrifugation, the cells were prewarmed at $37^{\circ} \mathrm{C}$, resuspended in internalization medium (added in a volume equal to that of the cellular pellet) and incubated for $5 \mathrm{~min}$ at $37^{\circ} \mathrm{C}$ (with mild agitation once per minute). The internalization was stopped by cooling on ice; the cells were then washed with icecold PBS containing $5 \mathrm{mg} / \mathrm{ml} \mathrm{BSA}$ and with ice-cold homogenization buffer. The cellular pellet was resuspended in homogenization buffer (approximately $3 \mathrm{ml}$ of buffer per $2 \mathrm{ml}$ of pellet), containing protease inhibitors $(0.2 \mathrm{mM} \mathrm{PMSF}, 1 \mu \mathrm{g} / \mathrm{ml}$ each of leupeptin, aprotinin and pepstatin A). The cells were homogenized by 10 passages through a stainless-steel ball homogenizer with a clearance of $0.02 \mathrm{~mm}$ (Industrial Tectonics Inc, Dexter, Michigan) and the homogenates were centrifuged for $15 \mathrm{~min}$ at $1,200 \times \mathrm{g}$. The resulting postnuclear supernatant (PNS) was collected, and was either shock frozen in liquid nitrogen and preserved in $-80^{\circ} \mathrm{C}$ or used immediately for further experiments.

\subsubsection{Purification of early endosomes from PC12 cells via density gra- dient centrifugation}

$1.5 \mathrm{ml}$ of post-nuclear supernatant was mixed with $1.5 \mathrm{ml}$ ice-cold $62 \%$ sucrose solution, containing $3 \mathrm{mM}$ imidazole- $\mathrm{HCl}, \mathrm{pH}$ 7.4. The mixture was then overlaid with $3 \mathrm{ml}$ each of ice-cold $35 \%$ and $25 \%$ sucrose solutions (also containing $3 \mathrm{mM}$ imidazole), the tube was filled up with homogenization buffer and then centrifuged for $90 \mathrm{~min}$ at $151,000 \times \mathrm{g}$ at $4^{\circ} \mathrm{C}$. The band at the interface between the $35 \%$ and 
$25 \%$ sucrose solutions, corresponding to enriched early endosomes, was isolated, its protein concentration was determined and the purified early endosomes were used in further experiments or shock frozen in liquid nitrogen and preserved in $-80^{\circ} \mathrm{C}$.

\subsubsection{Preparation of synaptic-vesicle-enriched LS1 fraction and cytosol from rat brains}

The following protocol was adapted from Huttner et al. (1983). Four adult rats ( 250 grams each) were decapitated and the brains were rapidly placed into icecold sucrose buffer (320 mM sucrose, 5 mM HEPES, pH 7.4). From this point on, the material was kept at $4^{\circ} \mathrm{C}$ throughout the preparation. The cerebrum cortices (without the brain stem and most of the midbrain) and the cerebelli were homogenized in $50 \mathrm{ml}$ sucrose buffer in a glass-Teflon homogenizer (using 10 up-and-down strokes at $900 \mathrm{rpm})$. The material was separated into 50-ml polycarbonate tubes and the cellular fragments were pelleted by centrifugation for 2 min at 2,000 $\times \mathrm{g}$. The pellets (containing mainly large cellular fragments, blood cells and mitochondria) were discarded and the supernatants (S1) were centrifuged further for $15 \mathrm{~min}$ at $7,800 \times \mathrm{g}$, to yield pellets $(\mathrm{P} 2)$ consisting mainly of synaptosomes, mitochondria and myelin. The pellets were washed (avoiding the relatively harder, brown-coloured mitochondrial component) in $30 \mathrm{ml}$ sucrose buffer each and recentrifuged for $15 \mathrm{~min}$ at $9,500 \times \mathrm{g}$. Supernatants were removed and each of the pellets $(\mathrm{P} 2)$ was resuspended in $15 \mathrm{ml}$ sodium buffer $(140 \mathrm{mM} \mathrm{NaCl}, 5 \mathrm{mM} \mathrm{KCl}, 10 \mathrm{mM}$ glucose, $5 \mathrm{mM}$ $\mathrm{NaHCO}_{3}, 1.2 \mathrm{mM} \mathrm{Na} \mathrm{HPO}_{4}, 1 \mathrm{mM} \mathrm{MgCl}$, $20 \mathrm{mM}$ HEPES, pH 7.4). The suspensions were pooled and centrifuged for 8 min at $11,200 \times$ g. All sodium buffer was removed and ice-cold distilled $\mathrm{H}_{2} 0$, containing $0.5 \mathrm{mM} \mathrm{PMSF}$, was added in a 4-fold higher volume than that of the pellet, to cause osmotic lysis of the pelleted synaptosomes. The suspension was homogenized (approximately 10 up-and-down strokes at maximum speed) and appropriate amounts of ice-cold 5X homogenization buffer (1.25 M sucrose, $15 \mathrm{mM}$ imidazole), containing protease inhibitors $(5 \mu \mathrm{g} / \mathrm{ml}$ of leupeptin, aprotinin and pepstatin A), were added to the homogenate. The synaptosomal membranes were pelleted for 20 min at $24,000 \times \mathrm{g}$ and the supernatant (an LS1-like fraction, enriched in synaptic vesicles) was collected. 


\subsubsection{Preparation of rat brain cytosol}

Approximately twenty adult rats were decapitated. The brains were rapidly placed into ice-cold sucrose buffer (320 mM sucrose, 5 mM HEPES, pH 7.4) and homogenized in sucrose buffer, containing $1 \mu \mathrm{g} / \mathrm{ml}$ pepstatin and $0.2 \mathrm{mM}$ PMSF, in a 50-ml glass-Teflon homogenizer (10 brains per $50 \mathrm{ml}$ sucrose buffer, 10 up-and-down strokes at $900 \mathrm{rpm}$ ). From this point on, the material was kept at $4^{\circ} \mathrm{C}$ throughout the preparation. The homogenate was centrifuged at $5000 \mathrm{rpm}$ using the SS-34 rotor (Sorvall) for $10 \mathrm{~min}$. The resulting pellet contained both the nuclei and the cell fragments (pink layer), as well as synaptosomes, myelin and mitochondria (yellow layer). Supernatant and part of the synaptosome pellet was isolated and centrifuged at 16,500 rpm in SS-34 rotor, for $15 \mathrm{~min}$. The resulting supernatant contained cytosol and small-sized organelles and was further cleared up by centrifugation at $340,000 \times \mathrm{g}$ for $30 \mathrm{~min}$. The cytosol was aliquoted, snap-frozen and stored at $-80^{\circ} \mathrm{C}$.

\subsubsection{Cell-free fusion and docking assay}

Cells were harvested, fluorescently-labeled marker was internalized to label the early endosomal population and postnuclear supernatant was prepared as described before. Briefly, cells were collected and washed with internalization medium, prewarmed, and incubated for 5 min with marker (10-kDa dextran labeled with Alexa 488 or Alexa 594, respectively; Molecular Probes) dissolved in internalization medium at a concentration of $1 \mathrm{mg} / \mathrm{ml}$ and added in a volume equal to that of the cellular pellet. After the internalization had been stopped by transfer on ice, the cells were washed three times with ice-cold PBS containing $5 \mathrm{mg} / \mathrm{ml} \mathrm{BSA}$ and were processed for the isolation of postnuclear supernatant.

Reaction mixtures contained, as final concentrations, $4 \mathrm{mg} / \mathrm{ml}$ PNSs $(2 \mathrm{mg} / \mathrm{ml}$ of Alexa 488-Dextran-labeled PNS and $2 \mathrm{mg} / \mathrm{ml}$ of Alexa 594-Dextran-labeled PNS, $2 \mathrm{mg} / \mathrm{ml}$ rat brain cytosol, $11.25 \mathrm{mM}$ Hepes, pH 7.0, $1.35 \mathrm{mM}$ magnesium acetate, $0.18 \mathrm{mM}$ DTT, $45 \mathrm{mM}$ potassium acetate, and $3.2 \mathrm{mM} \mathrm{ATP} / 26 \mathrm{mM}$ creatine phosphate/0.132 mg of creatine kinase (Roche, Basel, Switzerland) as an ATPregenerating system or 1500 units/ml of hexokinase (Roche, Basel, Switzerland) dissolved in $250 \mathrm{mM}$ glucose as an ATP-depleting system. The reaction mixtures 
were incubated for $45 \mathrm{~min}$ at $37^{\circ} \mathrm{C}$. Reactions were usually made at a final volume of $50 \mu \mathrm{l}$ and the volume was corrected by the addition of homogenization buffer.

Aliquots of the reaction solutions $(5-10 \mu \mathrm{l}$ from a $50 \mu \mathrm{l}$ reaction) were added onto coverslips (18-mm diameter; Marienfeld GmbH, Lauda-Knigshofen, Germany) in 12-well plates (each well containing $1 \mathrm{ml}$ of PBS) and centrifuged at 5,868 $\times \mathrm{g}$ in a Multifuge4 centrifuge (Heraeus Instruments, Hanau, Germany) for 45 min. TetraSpeck beads (200 nm diameter, dilution 1:100,000 in $1 \mathrm{ml}$ PBS; Molecular Probes) were pre-bound to the surface of the coverslips by centrifugation. Coverslips were analyzed by using a Zeiss Axiovert 200M fluorescence microscope (Jena, Germany) equipped with a 1.4 NA $100 \times$ objective and appropriate filters (see 2.2.17).

For the data analysis, a custom-written routine in Matlab (The Mathworks Inc., Natick, MA, USA) was used (see Appendix A2). Images were, first, high-pass filtered and thresholds of $6 \mathrm{AU}$ (green) and $4 \mathrm{AU}$ (red) above background were applied; all objects persisting above the thresholds (excluding single pixels) were then used in the analysis. The $\mathrm{x}$ and $\mathrm{y}$ coordinates of the intensity centres of the objects were determined and the shift between the images was corrected by use of the coordinates of a Tetraspeck bead (identified in the blue channel). The distances between the green and red objects were determined, and the percentage of green objects that were within $100 \mathrm{~nm}$ of red objects was calculated and considered as fused organelles. Endosomes whose red and green intensity centers were within 137.5-512.5 nm from each other were counted as docked. The value obtained for docking was also corrected for endosome density on the coverslip by subtracting a baseline due to the random distribution of the endosomes on the coverglass (the average percentage for the distances from 512.5 to $1012.50 \mathrm{~nm}$ ). The program can be found at Appendix A2.

\subsubsection{Immunoprecipitation}

PNS from wild type or knock-down cells was incubated for $45 \mathrm{~min}$ at $37^{\circ} \mathrm{C}$, under ATP-depleting conditions (1500 units/ml of hexokinase, dissolved in $250 \mathrm{mM}$ glucose). For the experiment in Figure 3.20, PNS was incubated in ATP-regenerating 
conditions (see 2.2.7) in presence or absence of $60 \mathrm{mg} / \mathrm{ml} \beta$-methyl-cyclodextrin for $30 \mathrm{~min}$ at $37^{\circ} \mathrm{C}$, with an additional $20 \mathrm{~min}$ in presence of $2 \mathrm{mM}$ NEM. Material was solubilized in a concentration of up to $0.7 \mathrm{mg} / \mathrm{ml}$ of protein by addition of icecold extraction buffer containing 2 mM PMSF. For immunoprecipitation from early endosomes, PNS was incubated under ATP-depleting conditions and loaded on a discontinuous sucrose gradient (see 2.2.4). The band enriched in early endosomes was isolated and its protein concentration was determined. Samples from the different cell lines were brought to the same protein concentration by addition of $25 \%$ sucrose buffer. Appropriate volume of $10 \times$ extraction buffer $(10 \times$ PBS, 50 mM EGTA, $50 \mathrm{mM}$ EDTA, 10\% Triton X-10) was added. Protein extraction was performed for $2 \mathrm{~h}$ at $4^{\circ} \mathrm{C}$, by rotating the samples on a rotary stage. To remove insoluble material, the extracts were centrifuged at $200,000 \times$ g for 25 min at $4^{\circ} \mathrm{C}$. Immunoprecipitation reactions were performed with the clear supernatants by adding either $2 \mu$ l of polyclonal anti-SNARE antibodies or $7 \mu \mathrm{l}$ of anti-SNARE monoclonal ascites fluids per $0.35 \mathrm{mg}$ of protein $(500 \mu \mathrm{l}$ reaction volume). As a negative control, no antibodies were added. The reaction tubes were incubated overnight at $4^{\circ} \mathrm{C}$, under continuous head-over-head rotation. Protein G Sepharose beads (Amersham Biosciences) were used to precipitate the antibody-protein complexes. Before use, Sepharose beads were washed three times (pelleting with 3 min-centrifugation steps at $700 \times \mathrm{g}$ ) and were resuspended in ice-cold extraction buffer, to a final suspension of $50 \%$ beads in extraction buffer. $50 \mu \mathrm{l}$ of the bead suspension was added to each immunoprecipitation reaction and samples were further incubated for $2 \mathrm{~h}$ at $4^{\circ} \mathrm{C}$. The beads were pelleted at $700 \times \mathrm{g}$ for $3 \mathrm{~min}$ and the supernatants were collected. Beads were washed three times with ice-cold extraction buffer and proteins were eluted with addition of $50 \mu \mathrm{l} 2 \times$ sample buffer. For the supernatants, protein elution was performed by the addition of appropriate volume $(125 \mu \mathrm{l})$ of $5 \times$ sample buffer. Samples were analyzed by SDS-PAGE and Western blotting.

The percentage of precipitation was determined using the following formula: intensity of precipitated protein band $\times 100 /$ intensity of starting material band, where starting material refers to the sum of the intensity of the precipitated protein and the intensity of its corresponding supernatant. The final amount of co- 
immunoprecipitated protein was calculated after correcting for the amount of precipitated protein (which was set to 100\%).

\subsubsection{Western blotting}

Samples were separated in a 10\% denaturating Tris/Tricine SDS polyacrylamide gel electrophoresis system, as described by Schägger and von Jagow (1987). The resolving gel consisted of 10\% bis-acrylamide (Rotiphorese Gel 30, Roth GmbH, Karlsruhe, Germany), 1 M Tris (pH 8.45), 0.1\% SDS, $10 \%$ glycerol; the stacking gel contained $4 \%$ bis-acrylamide, $1 \mathrm{M}$ Tris (pH 8.45), 0.1\% SDS. Ammonium persulfate and TEMED (N, N, N', N'-Tetramethylethylenen-diamine) were added for polymerization. Before loading, all samples were boiled for 5 min. $5 \mu \mathrm{l}$ PageRuler prestained protein ladder solution (Fermentas) were used in every blot, for approximate sizing of the proteins. Separation was performed in a discontinuous buffer system, with a $0.2 \mathrm{M}$ Tris ( $\mathrm{pH} 8.9$ ) solution in the tank and a $0.3 \mathrm{M}$ Tris ( $\mathrm{pH} \mathrm{8.45),0.03 \%} \mathrm{SDS}$ solution as the gel buffer. Subsequently, proteins were transferred to Protran nitrocellulose membrane (PerkinElmer Life Sciences, Boston, MA USA), via a semi-dry procedure in buffer containing $200 \mathrm{mM}$ Glycine, $25 \mathrm{mM}$ Tris (pH 7.4), 0.04\% SDS, $20 \%$ methanol. For the transfer, $45 \mathrm{~mA}$ were applied per blot for $1 \mathrm{~h}$ in a Biorad PowerPac 300 blotting apparatus. The membranes were blocked for $30 \mathrm{~min}$ at room temperature in blocking solution (PBS pH 7.4, 5\% non-fat milk powder, $0,1 \%$ Tween 20) and then incubated overnight with the appropriate dilutions of the primary antibody in blocking solution, at $4^{\circ} \mathrm{C}$. After 3 washes with blocking solution (10 min each), the blots were incubated with horseradish peroxidase-conjugated secondary antibodies (diluted 1:4000), for $1 \mathrm{~h}$ at room temperature. Protein bands were detected using the enhanced chemiluminescence (ECL) system (PerkinElmer LAS, Inc., Boston) on a FujiFilm LAS-1000 imaging station. The relative intensity of each band was calculated using a Matlab (Mathworks) code (designed by Silvio Rizzoli, European Neuroscience Insititute). The band of interest was manually selected; background intensity was calculated in the immidiate vicinity of the band and was substracted from the band's intensity value. 


\subsubsection{WB analysis for determination of protein expression levels}

Cells, grown to confluency, were washed twice with ice-cold Tris buffer (20 mM Tris, $150 \mathrm{mM} \mathrm{NaCl}, 1 \mathrm{mM} \mathrm{MgCl}$, protease inhibitors cocktail (Roche, Basel, Switzerland), $1 \mathrm{mM}$ PMSF, $0.2 \mathrm{mg} / \mathrm{ml}$ DNAse (Roche)) and collected in Tris buffer containing $1 \%$ Triton. Proteins were extracted for $90 \mathrm{~min}$ at $4^{\circ} \mathrm{C}$ and samples were centrifuged for $30 \mathrm{~min}$ at $16000 \times \mathrm{g}$. Protein concentration was determined using the BCA method (Pierce, Rockford, IL) and appropriate volumes of $2 \times$ sample buffer were added. Alternatively, cells homogenates from PNS preparation for each cell line were used. Equal protein amount were loaded to compare the levels of several proteins in the different cell lines. The protein levels for each cell line were normalized to the loading control (actin) and subsequently to the protein levels of the wild-type cells that were set to $100 \%$.

\subsubsection{Quantification of SNARE ammounts in PNS and early endosomes from PC12 and in LS1 fraction from rat brain homogenates}

Standard curves of purified recombinant proteins were separated by SDS-PAGE alongside with different amounts of PNSs, early endosomal and LS1 fractions, followed by immunoblot analysis. The protein amounts in each preparation were calculated by interpolation. Recombinant full length syntaxins 6 and 13, vtila, VAMP4 were kind gifts from Dr. Daniel Zwilling and syntaxin 1, synaptobrevin and SNAP25 were provided by Dr. Tabrez J. Siddiqui, MPI for Biophysical Chemistry, Gttingen, Germany.

\subsubsection{Analysis of EEA1 membrane recruitment}

PNSs from wild-type, control transfected and knock-down cells were incubated in presence of cytosol and ATP-regenerating conditions for $45 \mathrm{~min}$ at $37^{\circ} \mathrm{C}$. Reactions were centrifuged at $26,000 \times$ g. Supernatants were removed, pellets were resuspended in PBS-1\% Triton X-100 buffer and sonicated for 15 min. Sample buffer was added to the supernatants and pellets and the samples were analysed by immunoblotting, by loading equal amounts from pellet and supernatant. 


\subsubsection{Immunocytoshemistry}

PC12 cells were grown on poly-L-lysine coated coverslips. On the day of immunostaining, they were washed twice with ice-cold PBS and subsequently fixed for 45 min with $4 \%$ paraformaldehyde (PFA) in PBS ( $\mathrm{pH} 7.4)$. From this point on, all procedures were performed at room temperature. The free aldehyde groups were quenched by addition of $100 \mathrm{mM} \mathrm{NH}_{4} \mathrm{Cl}$ (in PBS) for $15 \mathrm{~min}$, the cells were briefly washed with PBS and permeabilized by washing with PBS containing $0.1 \%$ Triton $\mathrm{X}-100$ (3 times for $5 \mathrm{~min}$ ). The coverslips were incubated for $1 \mathrm{~h}$ with primary antibodies diluted 1:100 in PBS/0.1\% Triton X-100/1\% BSA. The coverslips were then washed 3 times with PBS/0.1\% Triton X-100 (5 min each) and incubated with Cy2, Cy3 or Cy5-labelled secondary antibodies, diluted at 1:100 in PBS/0.1\% Triton X100/1\% BSA, for 45 min. The coverslips were then washed briefly with PBS, 3 times for 5 min with high-salt PBS $(500 \mathrm{mM} \mathrm{NaCl}$ in $20 \mathrm{mM}$ sodium phosphate buffer, $\mathrm{pH}$ 7.4) and twice for 5 min with PBS followed by embedding in DakoCytomation fluorescent mounting medium.

\subsubsection{Uptake and trafficking assays}

Wild type, control transfected and knock-down cells were grown on poly-L-lysine coverslips to $60-80 \%$ confluency. To study the uptake of several endocytic markers, cells were incubated with either Alexa-594 labelled transferrin $(50 \mu \mathrm{g} / \mathrm{ml})$ or DiIlabelled LDL $(10 \mu \mathrm{g} / \mathrm{ml})$ or Alexa-594 labelled dextran 10kDa $(1 \mathrm{mg} / \mathrm{ml})$ or $20 \mu \mathrm{M}$ AM2-10 for $5 \mathrm{~min}$ at $37^{\circ} \mathrm{C}$ (all markers from Invitrogen, dissolved in OpitMEMglucose). Cells were then transferred on ice, washed twice with ice-cold PBS and fixed with 4\% PFA in PBS. To follow the recycling of transferrin, cells were incubated for 5 min at $37^{\circ} \mathrm{C}$ with Alexa-594-transferrin, were washed twice with pre-warmed medium, and further incubated in transferrin-free medium for 10 min at $37^{\circ} \mathrm{C}$. Cells were transferred on ice, washed and fixed. Uptake of the beta-subunit of Cholera toxin $(\mathrm{CTxB})$ was performed as following: Cells were incubated for 30 min at $4^{\circ} \mathrm{C}$ in Krebs buffer containing $10 \mu \mathrm{g} / \mathrm{ml}$ Alexa-594 labelled CTxB (Invitrogen). Unbound $\mathrm{CtxB}$ was washed away with $\mathrm{Ca}^{2+}$-free Krebs solution and the entry of $\mathrm{CtxB}$ into the cells was allowed by incubating the cells for 40 min at $37^{\circ} \mathrm{C}$ in $\mathrm{CTxB}$-free 
medium. Cells were transferred on ice, washed and fixed. Cells were visualised using a Zeiss Axiovert 200M fluorescence microscope equipped with a 1.4 NA $63 \mathrm{x}$ objective and the fluorescent signal was measured using custom-written routine in Matlab (The Mathworks Inc., Natick, MA, USA). Three independent experiments were performed and 10-15 cells were visualised in each experiment. The average fluorescence, corresponding to each different marker, was calculated for each cell type.

\subsubsection{Norepinephrine release}

The assay was performed according to Klenchin et al. (1998). Briefly, wild type PC12 and knock-down cells were grown for $24 \mathrm{~h}$, in $10 \mathrm{~cm}$-diameter dishes, containing 10 $\mathrm{ml}$ of complete medium supplemented with $0.5 \mathrm{mM}$ ascorbic acid and $4 \mu \mathrm{I} \mathrm{I}-\left[7,8{ }^{3} \mathrm{H}\right]$ noradrenaline (38 Ci/nmole, GE Healthcare). After washing with Krebs solution, control cells were incubated in $3 \mathrm{ml}$ of Krebs solution for 15 min at $37^{\circ} \mathrm{C}$, while stimulated cells were incubated in $3 \mathrm{ml}$ Krebs solution containing $50 \mathrm{mM} \mathrm{NaCl}$ and $90 \mathrm{mM} \mathrm{KCl}$. Supernatants were collected and the cells were lysed in $1 \mathrm{ml}$ PBS containing $1 \%$ Triton X-100, by intensive shaking. The amount of radioactivity contained in each sample was counted using a liquid scintillation analyzer (Canberra Packard, Schwadorf, Austria). The percentage of release was calculated by dividing the amount of radioactivity of the supernatant by the sum of the radioactivity in supernatant and the corresponding cell lysate.

\subsubsection{NGF-induced differentiation of PC12 cells}

Cells were grown on poly-L-lysine coverslips for 24,48 or $72 \mathrm{~h}$, in medium containing $100 \mathrm{ng} / \mathrm{ml}$ NGF (Sigma Aldrich). Cells were imaged in solution containing the styryl dye AM 2-10, using a Zeiss Axiovert 200M fluorescence microscope equipped with a 20x/0.30 Ph1 objective. The length of the longest neurite per field of view was measured and data was averaged for 10-20 cells per cell line and per time point, from three independent experiments. 


\subsubsection{Fluorescence Microscopy}

For epifluorescence microscopy, a Zeiss Axiovert 200M fluorescence microscope (Jena, Germany) equipped with a 1.4 NA $100 \times$ or $63 \times$ objective and a CCD camera with a $1317 \times 1035$ Kodak chip (pixel size $6.8 \times 6.8 \mu \mathrm{m}$, Princeton Instruments Inc., Trenton, NJ, USA) was used. Alexa 488 and Cy2 fluorescence (green channel) was detected with the excitation filter 480/40 HQ, the beamsplitter 505 LP Q and the emission filter 527/30 HQ. Alexa 594 and Cy3 fluorescence was detected using the excitation filter 560/55 HQ, the beamsplitter $595 \mathrm{LP} \mathrm{Q}$ and the emission filter 645/75 HQ. DiI fluorescence was detected using the excitation filter 545/30 HQ, the beamsplitter 570 LP Q and the emission filter 610/75 HQ. Blue fluorescence (dapi) was detected using the excitation filter 350/50 D, the beamsplitter 400 DCLP and the emission filter 460/50 D. Cy5 fluorescence was detected using the excitation filter 620/60 HQ, the beamsplitter 660 LP Q and the emission filter 700/75 HQ. All filters were purchased from Chroma, Rockingham, VT, USA. Image acquisition was performed using Images were processed using METAMORPH (Universal Imaging Corporation, West Chester, PA, USA).

Confocal images were acquired with a Leica TCS SP5 confocal microscope (Leica Microsystems GmbH, Mannheim, Germany) using a 63×, 1.4 numerical aperture HCX PL APO objective, and the LAS AF 1.5.1 software. Confocal images of the EEA1 distibution on GFP-Rab5-Q79L-positive endosomes and STED images were acquired using a TCS SP5 STED microscope, equiped with an $100 \times, 1.4$ NA objective (Leica). Acquisition was performed using the sequential line mode, so that the images from two different channels were perfectly aligned. 


\section{3 \\ RESULTS}

\subsection{Specificity in SNARE function}

\subsubsection{Synaptobrevin, the most abundant endosomal SNARE, is not in- volved in early endosomal fusion}

As discussed in the Introduction, early endosomal (syntaxins 13 and 6, vti1a and VAMP4) and exocytic (syntaxin 1, SNAP-25, synaptobrevin) SNAREs co-reside on the membrane of the early endosomal compartment in PC12 cells. However, they show no functional overlap: the former SNARE set promotes homotypic early endosomal fusion while the latter regulates exocytosis. Cleavage of SNAP-25 with $\mathrm{BoNT} / \mathrm{E}$, or of syntaxin 1 with BoNT/C1, as well as competition experiments using cytosolic fragments of exocytic SNAREs, do not inhibit fusion of early endosomes (Brandhorst et al., 2006; Rizzoli et al., 2006). Additionally, selective cleavage of the three exocytic SNAREs blocks exocytosis (Humeau et al., 2000), showing that the early endosomal SNAREs cannot substitute for the function of their exocytic counterparts in this reaction. Nevertheless, the members of the two functionally distinct SNARE sets can interact with each other and can promiscuously associate in genuine cis-SNARE complexes (Bethani et al., 2007). More specifically, the endosomal Q-SNAREs strongly bind to the exocytic R-SNARE synaptobrevin, and they even seem to prefer the exocytic, non-cognate SNARE over their cognate partners, in cis-associations, as shown in Figures 1.6.

Even though addition of recombinant synaptobrevin does not impair early endosomal fusion in vitro (Brandhorst et al., 2006), the strong association of the protein with early endosomal SNAREs led me to further investigate the influence 

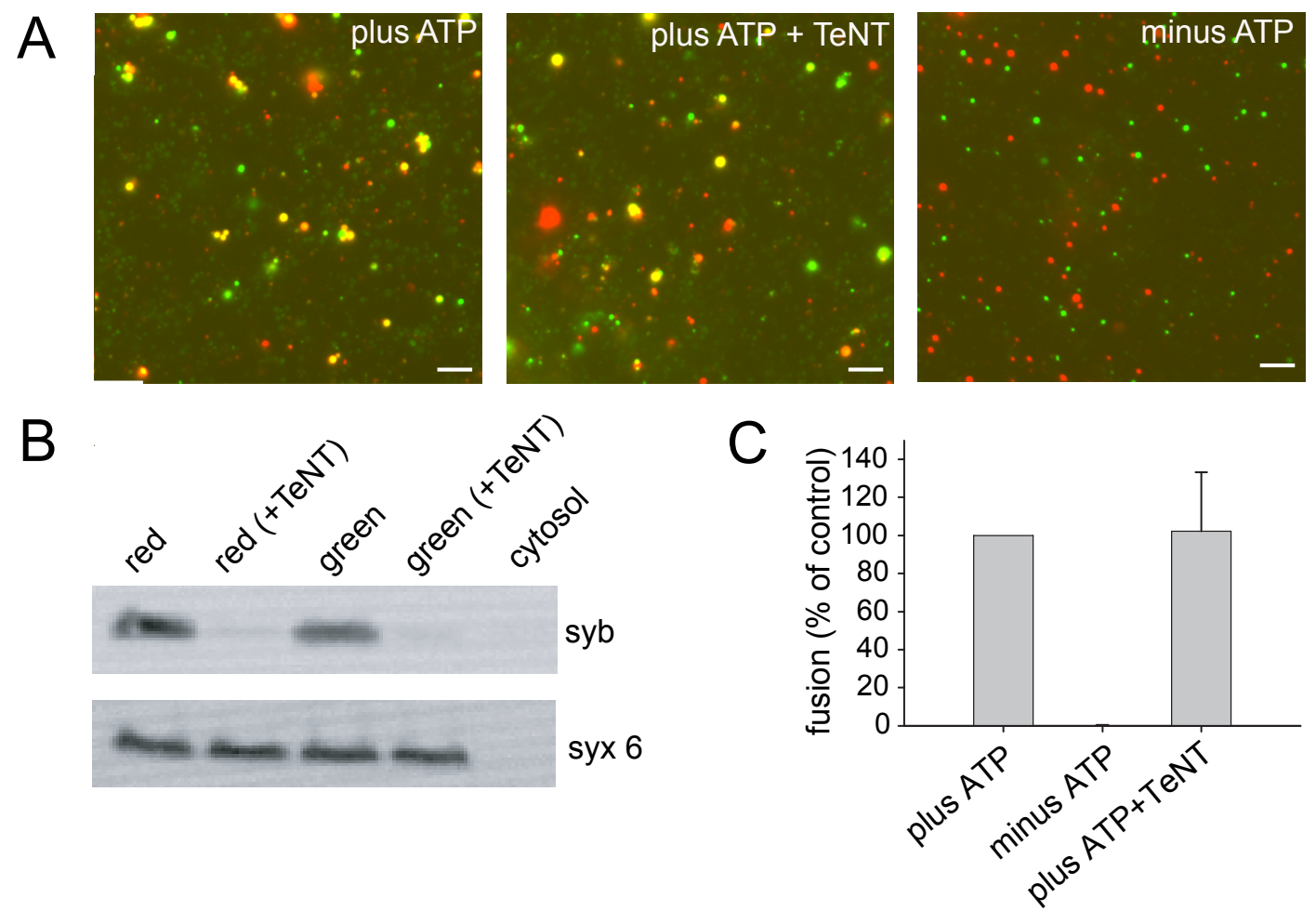

Figure 3.1: Synaptobrevin is not involved in homotypic fusion of early endosomes. PC12 cells were loaded for 5 min with Alexa 488-labeled (green) or Alexa 594-labeled (red) dextran. Cells were then homogenized and post-nuclear supernatant (PNS) containing the labeled organelles was isolated. The two differentially labeled PNSs were incubated in the presence of cytosol and in the absence or presence of an ATP-regenerating system, for 45 min at $37^{\circ} \mathrm{C}$. A) Representative images of fusion reactions that were carried out in the presence of ATP (left panel), after preincubation of the two endosome populations with tetanus toxin (middle panel) or in the presence of an ATP-depleting system (right panel). Scale bar: $4 \mu \mathrm{m}$. B) Labeled postnuclear fractions were incubated separately in presence of cytosol and an ATP-regenerating system (as in A), either in absence or presence of the recombinant light chain of tetanus toxin. Fractions were analyzed by immunoblotting for synaptobrevin (syb) and syntaxin 6 (syx 6, as loading control). C) Quantification of homotypic fusion of early endosomes under the three conditions. Values are means \pm s.e.m. of four independent experiments. Values are normalized to fusion in the control population.

of this protein on endosomal fusion by specifically eliminating its function by use of tetanus neurotoxin. Synaptobrevin was specifically cleaved by tetanus toxin in the post-nuclear supernatant (PNS) of PC12 cells and the ability of early endosomes lacking the full-length protein to perform homotypic fusion was tested. 'Green' and 'red'-labeled PNS from PC12 cells was prepared as described in 2.2.3 and the two 
endosome populations were incubated with the recombinant light chain of tetanus toxin for $7 \mathrm{~min}$ at $37^{\circ} \mathrm{C}$, before combining them. The reaction was incubated in the presence of cytosol and ATP for $45 \mathrm{~min}$ at $37^{\circ} \mathrm{C}$, as described in 2.2.7. Control reactions that did not contain tetanus toxin were performed in parallel, either in the presence or in the absence of ATP. The tetanus-treated PNS was analyzed by immunoblotting for the presence of synaptobrevin and as shown in Figure 3.1, the efficiency of the toxin cleavage was high, with barely detectable levels of uncleaved synaptobrevin left in the preparation. As expected, ATP-dependent early endosomal fusion was not affected by synaptobrevin cleavage, excluding a role for this protein in this fusion process. This is in agreement with previous observations from non-neuronal cells where tetanus toxin-mediated cleavage of cellubrevin did not affect fusion of early endosomes (Link et al., 1993).

\subsubsection{Cognate and non-cognate SNAREs occupy common microdomains on the endosomal membrane}

Since it is clear that there is no functional crosstalk between the early endosomal and the exocytic SNAREs, the ability of these SNAREs to promiscuously associate raises the question on how the functional specificity of SNARE molecules is regulated. What are the cellular mechanisms that ensure that only cognate SNAREs interact with each other for a specific fusion process, even though their association in cis is possible? A simple hypothesis is that the spatial organization of the different SNARE sets on the endosomal membrane contributes to their functional differentiation. It is conceivable that cognate SNAREs segregate in membrane microdomains that exclude non-cognate partners and that the observed interactions between endosomal and exocytic SNAREs occur only at the neighboring borders of these clusters, in agreement with previous reports on syntaxins 1 and 4, as well as syntaxins 3 and 4 (all participating in separate SNARE complexes, Low et al. (2006); Sieber et al. (2006)).

Taking advantage of antibodies specific for the early endosomal and exocytic SNAREs, I checked by means of fluorescent microscopy the organization of different SNARE molecules on the endosomal membrane. Since early endosomes from 


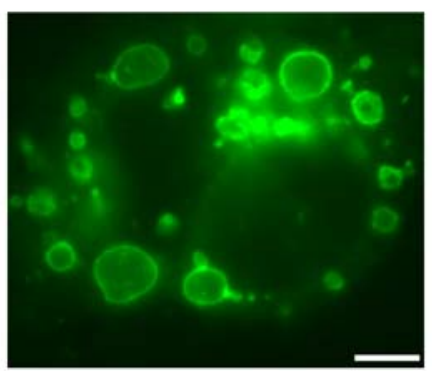

Figure 3.2: Formation of enlarged endosomes in PC12 cells transfected with GFPRab5-Q79L. PC12 cells were transfected with a plasmid expressing GFP-Rab5-Q79L. Forty-eight hours post-transfection, cells were fixed and imaged by use of a Zeiss Axiovert 200M fluorescence microscope. The GFP-bound Rab5 variant was observed on vesicular structures of $15 \mathrm{~mm}$, which correspond to enlarged early endosomes. Scale bar: $5 \mu \mathrm{m}$

PC12 cells are too small $(\sim 200 \mathrm{~nm})$ to allow the visualization of discrete domains on their membrane, I applied the following strategy. I transfected PC12 cells with a constitutively active mutant of the endosomal GTPase Rab5 (Rab5-Q79L) that was tagged with GFP. Due to the positive regulatory role of Rab5 in homotypic early endosomal fusion, transfection of this mutant results in the formation of enlarged endosomes (up to $5 \mu \mathrm{m}$ ) due to increased homotypic fusion activity (Stenmark et al., 1994) (Figure 3.2). No severe changes in cell viability and endosomal trafficking are observed under these conditions. The cells were then immunostained for pairs of SNAREs belonging to the same or different functional sets and visualized using confocal microscopy. Cells containing GFP-positive enlarged endosomes were selected and confocal sections were acquired around the equator of the organelles. SNAREs exhibited a pattern of punctuate staining, suggestive of membrane microdomain organization, in contrast to the more diffuse distribution of GFP-Rab5 (Figure 3.3). To measure the extent of colocalization between the domains exhibited by different SNARE pairs, the images from the three different channels (green for the GFP-Rab5, red for proteins immunostained with a Cy3-labeled secondary antibody and blue for proteins immunostained with a Cy5-labeled secondary antibody) were aligned, the intensity pattern of the fluorescence along the endosomal membrane was calculated for each channel (graphs in Figure 3.3) and correlation analysis was 

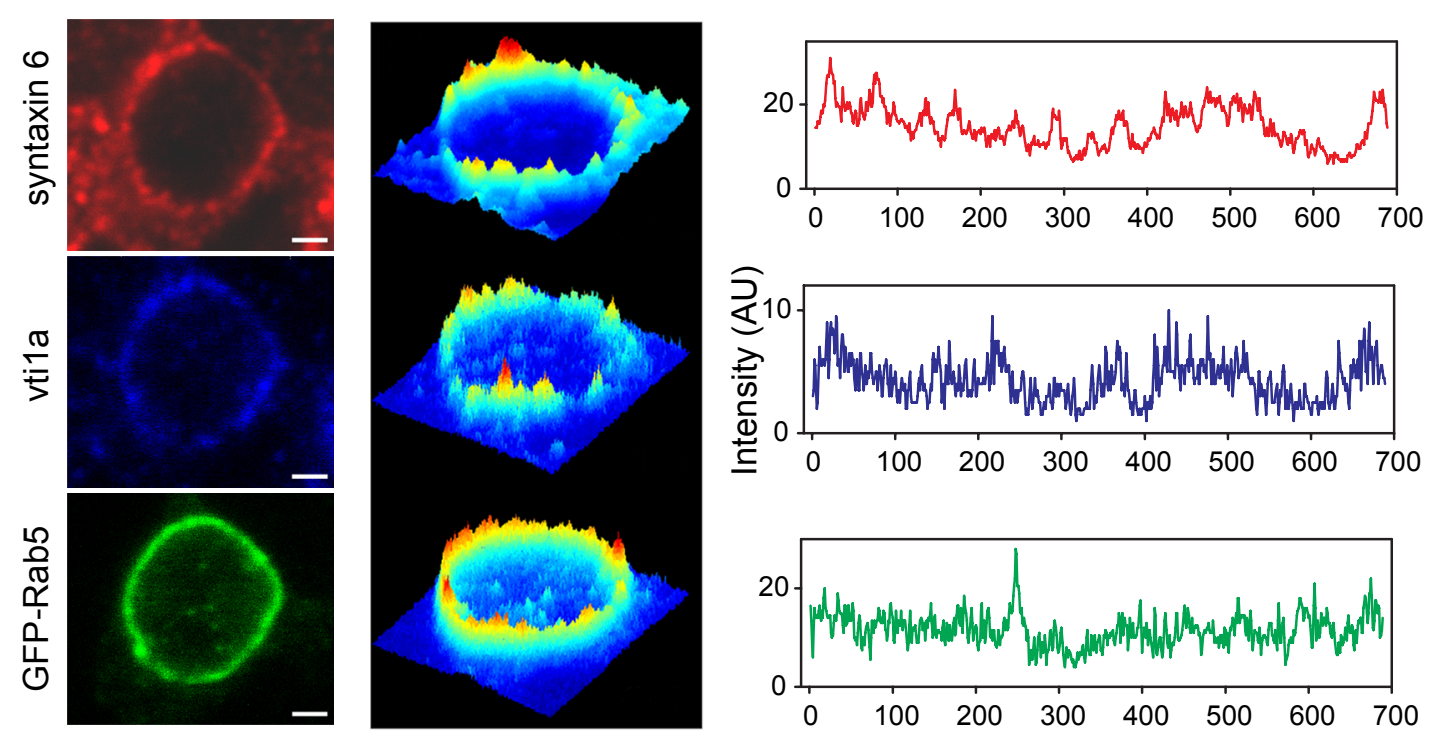

Figure 3.3: Intensity profiles along the membrane of an enlarged endosome co-stained for a pair of endosomal SNAREs. PC12 cells expressing the GFP-Rab5-Q79L (green) were stained for syntaxin 6 (red channel) and vtila (blue channel), and imaged using confocal fluorescence microscopy. Scale bar: $1 \mu \mathrm{m}$. The intensity images are plotted as surfaces in pseudocolor (center). Note that the SNAREs are found in domains, which largely correlate. The intensity profiles of syntaxin 6 (red), vtila (blue) and GFP-Rab5-Q79L (green) signals along the endosomal membrane of the endosome are also shown. These intensity valued were used for the calculation of the correlation coefficient.

performed. As a positive control, the correlation between the signals of co-staining of single SNAREs with Cy3- and Cy5-conjugated secondary antibodies was used. The negative control for each SNARE corresponds to the correlation coefficient between the intensity profile in the red channel and the intensity profile in the blue channel, placed in reverse order (mirrored, Figure 3.4). All SNARE pairs (cognate and non-cognate) revealed a similarly high degree of correlation (with the exception of the SNAP-25/vtila pair), suggesting an overlap in the spatial distribution of membrane SNARE microdomains, thus excluding that SNARE pairing specificity is regulated by membrane segregation of non-cognate partners. 


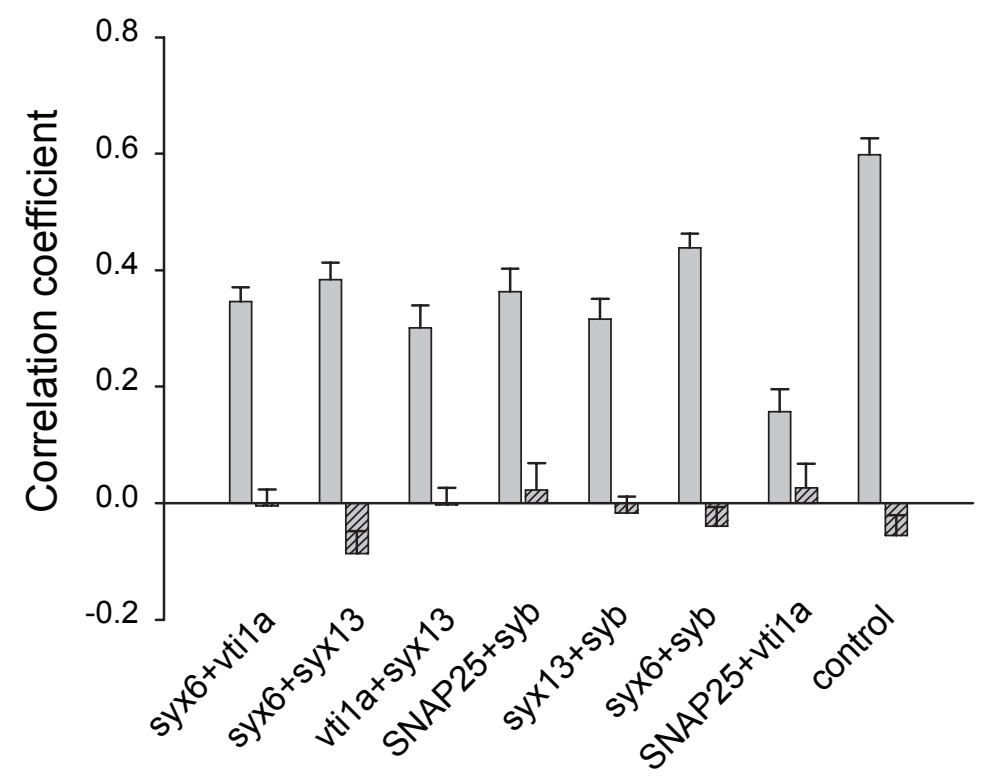

Figure 3.4: The cognate and non-cognate SNAREs largely colocalize in microdomains on the endosomal membrane. Correlation between the staining of different pairs of SNAREs on the endosomal membrane. The correlation coefficient for different SNARE pairs was calculated as described in the Material and Methods. Values are means \pm s.e.m. of three independent experiments with 10 - 15 analyzed endosomes. The striped bars correspond to the negative controls.

\subsubsection{Mass-action governs SNARE interactions}

SNARE interactions exhibit high promiscuity not only in vitro (Fasshauer et al., 1999), but also in the context of intracellular membranes, as already discussed for the sets of early endosomal and exocytic SNAREs (Bethani et al., 2007). A striking observation concerning membrane interactions is the high degree of preference that certain SNAREs exhibit for non-cognate over their cognate partners. For example, syntaxin 13 and syntaxin 6 interact much stronger with the non-cognate synaptobrevin than their other early endosomal partners in three different preparations (PC12-cell derived PNS and enriched endosomes, as well as in LS1 fraction from rat brain homogenates). In addition, all SNAREs (with the addition of the late endosomal proteins syntaxin 7 and vti1b) were present in the precipitates of synaptobrevin (with the exception of the R-SNARE VAMP4, see Introduction).

What is the reason for the strong promiscuous association of synaptobrevin with 
a variety of SNARE molecules? Is there a regulatory mechanism that favors certain associations in cis or is it the relative availability of the proteins that determines the kind and the amount of complexes formed? To address this issue, I determined the amounts of exocytic and early endosomal SNAREs in purified early endosomes, as well as in PNS from PC12 cells and a synaptic-vesicle enriched fraction from rat brain homogenates. Purified recombinant SNARE proteins were used to generate standard curves, after determining the linear range for each of them in Western blot detection. Different amounts of early endosomes, PNS and brain homogenate (varying between 1-5 $\mu \mathrm{g}$ ) were separated by SDS-PAGE alongside with the standard curves of recombinant proteins, Western blot analysis was performed and the SNARE protein content of each preparation was calculated by interpolation. From the data presented in Table 3.1, it is apparent that synaptobrevin outnumbers all other immunoprecipitated SNARE molecules (excluding SNAP-25), explaining its high involvement in cognate and non-cognate associations. Taking into consideration the low amounts of the endosomal R-SNARE VAMP4, it is not surprising that the endosomal Q-SNAREs are mainly engaged in cis-complexes with the significantly more abundant synaptobrevin. It should be mentioned that the most abundant protein in all preparations proved to be the other exocytic SNARE SNAP25. Indeed, SNAP-25 was present in the precipitates of both exocytic (syntaxin 1, synaptobrevin) and endosomal (syntaxins 13, 6 and VAMP4) SNAREs from purified endosomes (Bethani et al., 2007), emphasizing the correlation between promiscuity in complex formation and SNARE concentration. Nevertheless, the lack of an immunoprecipitating antibody that recognizes SNAP-25 engaged in complexes did not allow for studying its direct involvement in SNARE associations by checking its co-immunoprecipitating partners. However, the quantification of the SNARE concentrations did confirm that SNARE interactions in cis are determined, at least partially, by the relative amounts of SNAREs present on the membrane plane.

To further substantiate this hypothesis, I generated a simple Monte Carlo model describing SNARE associations on the endosomal membrane (in collaboration with Dr. Silvio Rizzoli, European Neuroscience Institute, Göttingen, see Appendix A1). We studied the complex formation of the two R-SNAREs synaptobrevin and 
Table 3.1: Quantification of exocytic and early endosomal SNAREs in different preparations. Standard curves of purified recombinant proteins were separated by SDS-PAGE alongside with different amounts of PNSs, early endosomal and LS1 fractions, followed by immunoblot analysis. The protein amounts in each preparation were calculated by interpolation. Here the amount in $\mathrm{pM}$ is presented. Means \pm s.e.m. from $3-5$ independent measurements are shown.

\begin{tabular}{lccc}
\hline \multicolumn{1}{c}{ pM } & LS1 & Early endosomes & PNS \\
\hline SNAP-25 & $471 \pm 24.7$ & $2,496 \pm 226$ & $739 \pm 40$ \\
Syntaxin 1 & $7.44 \pm 0.87$ & $2.94 \pm 0.55$ & $2.01 \pm 0.32$ \\
VAMP 2 & $127 \pm 9.20$ & $37.89 \pm 1.65$ & $6.77 \pm 2.99$ \\
Syntaxin 13 & $0.58 \pm 0.06$ & $3.32 \pm 0.40$ & $1.29 \pm 0.32$ \\
Syntaxin 6 & $0.46 \pm 0.07$ & $5.44 \pm 0.19$ & $2.59 \pm 0.09$ \\
Vti1a & $0.18 \pm 0.03$ & $0.68 \pm 0.16$ & $0.26 \pm 0.02$ \\
VAMP 4 & $0.015 \pm 0.005$ & $0.43 \pm 0.03$ & $0.28 \pm 0.04$ \\
\hline
\end{tabular}

VAMP4, with the acceptor complexes formed either by the endosomal Q-SNAREs (syntaxin 13/vtila/syntaxin 6) or by the exocytic Q-SNAREs (syntaxin 1/SNAP25). All SNAREs were inserted in their correct stoichiometry (according to Table 3.1): VAMP4 (five copies), early endosomal Q-SNARE complex (eight, limited by the low copy number of vtila), synaptobrevin (439) and the exocytic Q-SNARE complex (34, limited by the number of syntaxin 1 molecules). The density of the SNAREs per unit of surface was adjusted using synaptic vesicles as model for which such quantitative data are available (Takamori et al. (2006), Figure 3.5). We also assumed that the acceptor sites are permanently stable. We allowed the R-SNAREs to mix and interact freely with the QaQbQc acceptors for 1000 iterations and we then measured the amount of cognate and non-cognate complexes formed. Once a complex was formed, it persisted throughout the simulation, mimicking the experimental conditions used in the immunoprecipitation experiments, where NSF function was inhibited. Collision of the cognate partners resulted always in SNARE complex formation, whereas the probability of complex formation per collision for 
non-cognate interactions varied between 1 and 1:1000. In other words, we varied the fold difference in affinity for formation of cognate and non-cognate complexes between 1 and 1:1000.

Synaptobrevin dominated over VAMP4 for the interaction with the endosomal Qacceptor complex, when the probabilities of the two R-SNAREs to form complexes were comparable. Only when the affinity of VAMP4 reached 150-fold over synaptobrevin (a possibility that seems far from physiological conditions), endosomal cognate complexes outnumbered the non-cognate. Nevertheless, as the number of the endosomal QaQbQc acceptor sites is higher than the number of available VAMP4 molecules, some synaptobrevin-containing complexes were still able to form even when the synaptobrevin affinity approached to 0 (Figure 3.5, upper graph). Finally, VAMP4 can only engage minor amounts of syntaxin 1/SNAP-25 into complex, even when its affinity equals the one of synaptobrevin, clearly demonstrating that SNARE concentrations determine non only which complexes can form but also which cannot (Figure 3.5, lower graph).

Interestingly, while testing for the validity of the simulation, we noticed that the model's outcome highly correlated with the in vitro co-immunoprecipitation results. We calculated the percentage of each endosomal Q-SNARE associated with either synpatobrevin or VAMP4 in our model when the difference between their probabilities of complex formation was only a few fold. The number of sx13/vti1a/sx6 complexes 'pulled down' was $\sim 0.5$ for VAMP4 and $\sim 7$ for synaptobrevin. This translated into VAMP4 'pulling down' $\sim 1.3 \%$ of syntaxin $13,0.8 \%$ of syntaxin 6 and $\sim 6 \%$ of vtila (as they are present in copy numbers of 38, 63 and 8, respectively); these numbers correlated perfectly with the fact that vtila was the only cognate SNARE for which measurable amounts were found in the VAMP4 precipitates (although only in the LS1 fraction). Similar was the case for synaptobrevin which 'immunoprecipitated' $\sim 18 \%$ of syntaxin $13, \sim 11 \%$ of syntaxin 6 and $\sim 7 \%$ of vti1a. The numbers agree perfectly with the amounts of SNAREs biochemically detected in synaptobrevin precipitate, with the only large deviation ( $\sim 6$-fold) being for vtila. This discrepancy may be explained by an involvement of vtila isoforms (such as vti1b) in the non-cognate complexes in real endosomes. 

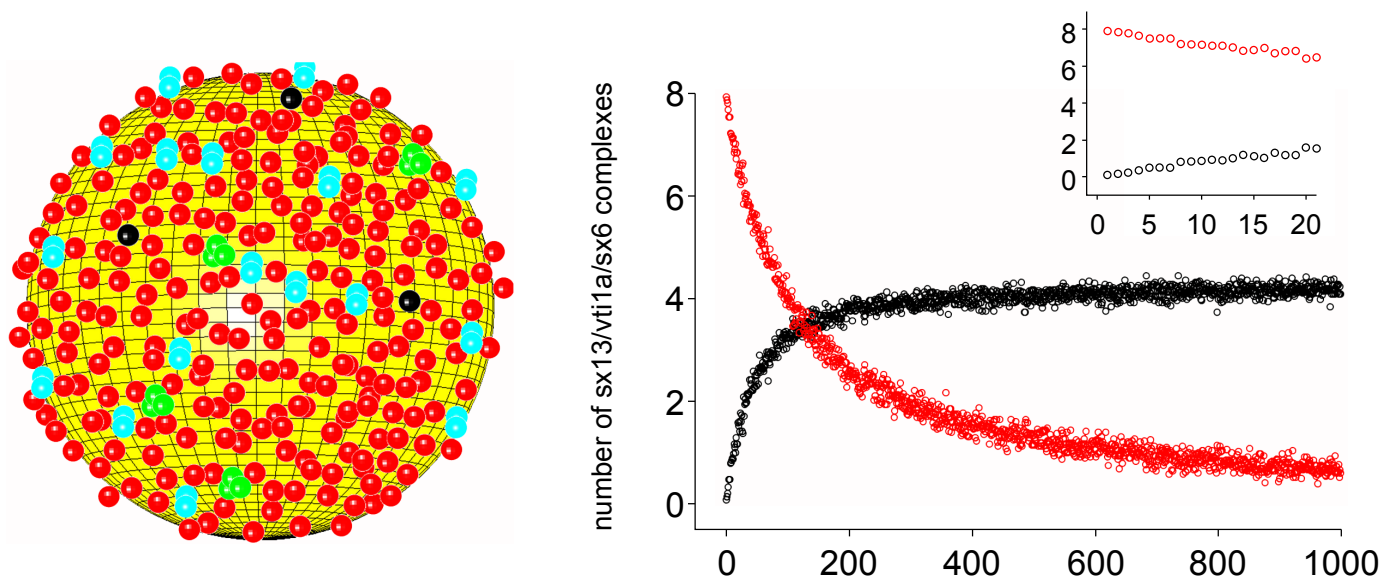

\section{Synaptobrevin (439) \\ VAMP4 (5)}

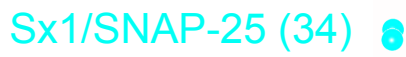

\section{Sx13/Vti1a/Sx6 (8) 8}

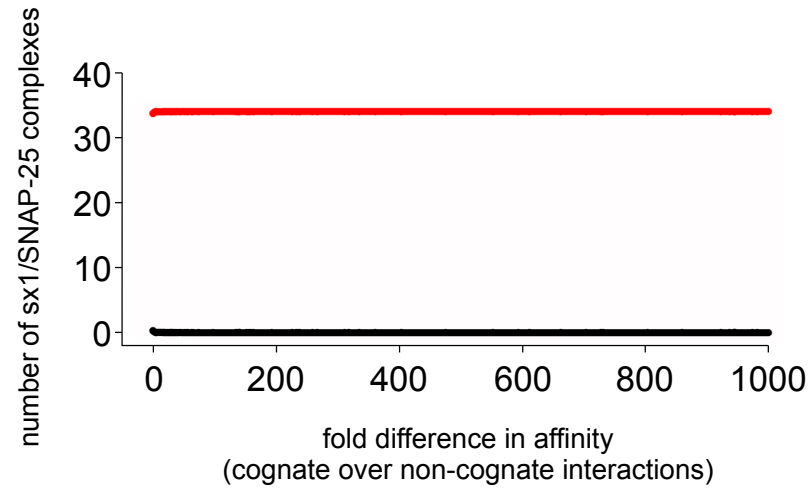

Figure 3.5: Mass action determines cis-complex formation. We simulated the behavior of SNAREs in a model endosome, containing synaptobrevin, VAMP4, the early endosomal Q-SNARE acceptor complex (syntaxin 13/vti1a/syntaxin 6) and the exocytic Q-SNARE acceptor complex (syntaxin 1/SNAP-25). The levels of the different elements are shown in parenthesis; the graphic description of the model is provided to give an impression of the different SNARE densities. We varied the affinity of R-SNAREs for their non-cognate complex acceptors between 1 and 1/1000 and then counted the number of both cognate and non-cognate complexes. The fold difference in affinity is indicated on the $x$-axis. Upper graph: The number of complexes containing the early endosomal Q-SNARE acceptor (synaptobrevin-containing complexes, red; VAMP4-containing complexes, black). As synaptobrevin is the non-cognate partner here, its affinity decreases from left to right. The inset zooms on the first 20 points of the curves. Lower graph: The number of complexes containing the exocytic Q-SNARE acceptor. As VAMP4 is the non-cognate partner here, its affinity decreases from left to right. Note that, due to the differences in concentration, essentially no VAMP4-containing (non-cognate) complexes form, even when the affinity is 1 for both the R-SNAREs (the left-most point). 
Summarizing, the data presented in this section favor the view that the number and the identity of the SNARE complexes formed on a certain membrane platform mainly depend on the relative amounts of the available SNAREs. Looking more specifically at the membrane of PC12-cell derived early endosomes, stoichiometric imbalances among exocytic and endosomal SNAREs seem to favour the formation of non-cognate SNARE complexes, and therefore raising the question of regulation in SNARE function specificity.

\subsubsection{Specificity of SNARE pairing is ensured by two co-operative mech- anisms}

\section{Lateral segregation of SNAREs at contact/fusion sites between endo- somes}

The high colocalization among non-cognate SNAREs, together with the abundance of non-cognate complexes on the endosomal membrane excluded the possibility of proof-reading mechanisms that function in cis and determine the identity of SNARE complexes. Since fusion requires the specific association of cognate SNAREs in trans, the function of intrinsic membrane mechanisms that regulate SNARE pairing in trans-configuration is possible.

Furthermore, it is generally accepted that intracellular membrane fusion is preceded by the establishment of docking contacts between the opposing membranes. Docking of early endosomes involves the formation of supramolecular complexes, that crosslink the membranes and contain the early endosome-specific GTPase Rab5 and its effectors (Zerial and McBride, 2001). Interestingly, these proteins have been shown to interact with early endosomal SNAREs, concentrating this way the cognate molecules at the prospective fusion sites and thus possibly excluding the noncognate, unnecessary partners.

To study the distribution of SNAREs at docking sites between endosomes, I took advantage of the enlarged organelles formed upon transfection of cells with the Rab5Q79L (Figure 3.2). Two days post-transfection, pairs of closely apposed endosomes were detectable. They are likely to represent pre-fusion intermediates, as constricted endosomes indicative of recent fusion of large organelles were also 
relatively frequently observed (Figure 3.6D). The cells containing the enlarged endosomes were immunostained for synaptobrevin, and despite the low frequency of endosome contact sites, I was able to detect three pre-fusion intermediates from three independent experiments of synaptobrevin-stained preparations. Interestingly, in all cases, the endosomal contact sites seemed to be devoid of synaptobrevin (Figure 3.6A-C). Conversely, an interface that was co-immunostained for synaptobrevin and syntaxin 6 contained comparatively more endosomal SNARE than synaptobrevin (Figure 3.6C). It can therefore be concluded that despite the overlapping distribution and the interactions among non-cognate partners, SNAREs appear to segregate prior to fusion. At the docking/pre-fusion sites of contact, non-cognate SNAREs seem to be excluded, favoring interactions among the cognate partners and contributing to SNARE pairing selectivity.

\section{Only the synergistic function of two regulatory mechanisms ensures SNARE specificity}

Is the special segregation of SNARE molecules occurring during docking the only mechanism promoting cognate SNARE associations in trans? In experiments that were performed by Prof. Thorsten Lang (University of Bonn) and were presented at Bethani et al. (2007), the existence of a selectivity filter that favors cognate SNARE interactions in trans was shown. Using inverted lawns of PC12 cells, the ability of membrane-bound SNAREs to associate with externally-added recombinant SNARE molecules was studied. It was clearly demonstrated that recombinant early or late endosomal SNAREs cannot compete with the recombinant exocytic SNAREs for the binding to the endogenous exocytic SNAREs present on the membrane sheets.

These data clearly demonstrated that the plasma membrane SNAREs distinguish between cognate and non-cognate partners, when the later are presented in a transconfiguration. The efficiency, though, of such a proof-reading mechanism proved to be weak, as demonstrated in my Master's thesis and published in Bethani et al. (2007). When PC12-cell-derived PNS was incubated with saturating amounts of recombinant, myc-tagged synaptobrevin, endobrevin or VAMP4, cognate and noncognate complexes were formed with similar efficiency, implying that the proof- 


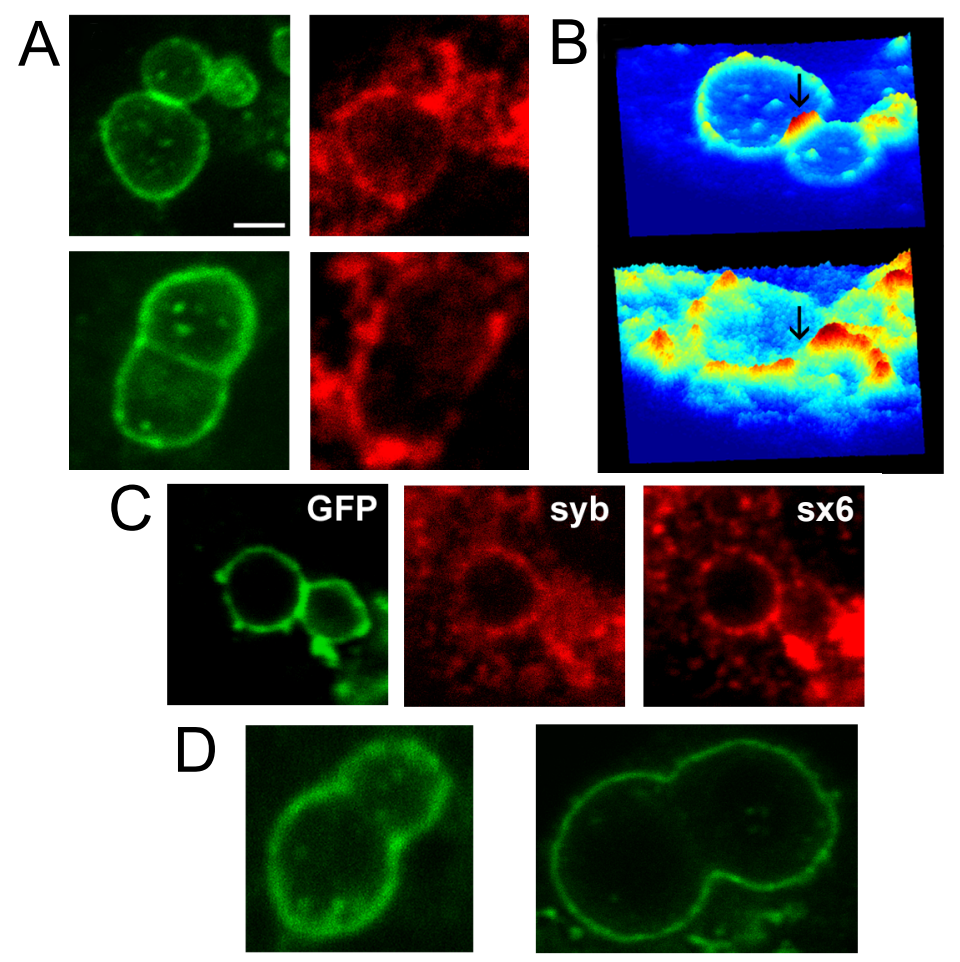

Figure 3.6: Synaptobrevin appears to 'avoid' the interfaces between endosomes. PC12 cells were transfected with a plasmid expressing GFP-Rab5-Q79L and immunostained for synaptobrevin. A) Two endosome pairs showing clear interfaces; green, Rab5-GFP; red, synaptobrevin. Scale bar: $1 \mu \mathrm{m}$. Note that synaptobrevin is excluded from the fusion interface. B) Threedimensional view of the endosomes in the top panels of A. Top, Rab5-GFP. Bottom, synaptobrevin. Arrows point to the interface. C) Endosome pair similarly stained for synaptobrevin (middle) and syntaxin 6 (right). D) The fate of the endosomes pressing against each other is difficult to predict; they most likely fuse, as we relatively often see constricted (i.e. post-fusion) structures.

reading mechanism shown to operate in trans cannot alone ensure the specificity of SNARE-mediated fusion.

Therefore, two mechanisms seem to mediate the specificity in trans-SNARE pairing in intracellular fusion. First, the existence of a selectivity filter that favors cognate associations in trans configuration and second, the enrichment of the cognate and/or the de-enrichement of the non-cognate SNAREs, resulting in a change of local SNARE concentrations at the prospective fusion sites. Nevertheless, none of them separately seems to be able to regulate SNARE specificity. To test 

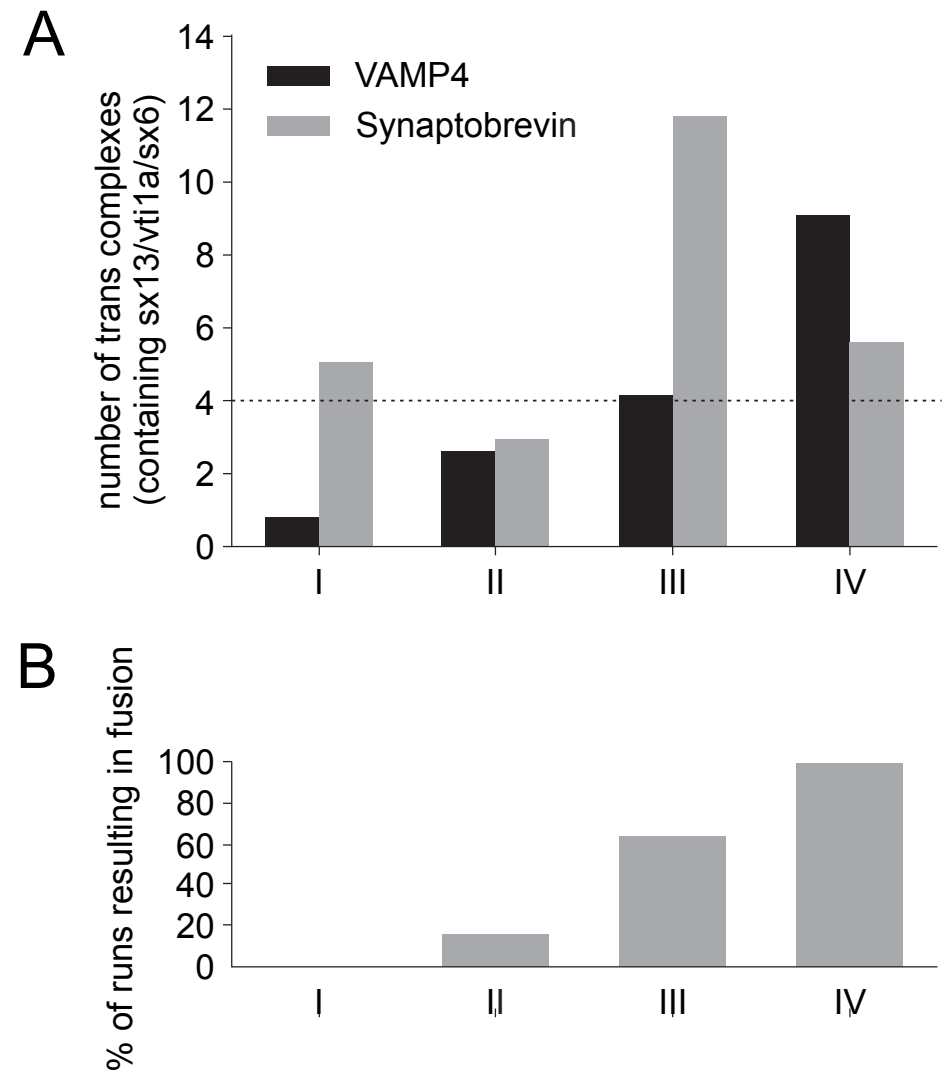

Figure 3.7: Fusion specificity is achieved by synergistically operating mechanisms. We simulated the behavior of two interacting endosomes of $\sim 233 \mathrm{~nm}$ diameter, each containing 25 VAMP4 molecules, 40 early endosomal Q-SNARE acceptor complexes (syntaxin 13/ vtila/syntaxin 6), 2195 synaptobrevin molecules and 170 exocytic Q-SNARE acceptor complexes (syntaxin 1/SNAP-25). We allowed the SNAREs from the two endosomes to interact in the interface between them for 1000 iterations. All trans-complexes that formed remained stable until the end of the simulation. Cis-complexes formed as well, but were always disengaged before the start of the next iteration. A) We monitored the number of trans-complexes containing the early endosomal Q-SNAREs (with either VAMP4, shown in black, or synaptobrevin, shown in gray). A 10-fold preference for cognate complex formation was modeled. Four models were used: I, random distribution of both exocytic and endosomal SNAREs - II, 10-fold de-enrichment of exocytic SNAREs from the interface - III, three-fold enrichment of endosomal SNAREs in the interface and IV, both a de-enrichment of exocytic SNAREs and an enrichment of the endosomal ones. B) Formation of at least four VAMP4-containing complexes was required to consider the two endosomes as fused. The average percentage of fused pairs for $\sim 3000$ simulations is shown.

whether these two mechanisms are indeed sufficient to ensure specificity and to evaluate their degree of co-operativity, we ran Monte Carlo simulations of the 
trans complexes formed at the interfaces of two opposing endosomes. SNAREs were inserted in their stoichiometric proportions as before, and they were allowed to interact at the interface of two endosomes for 1000 iterations, with a 10-fold preference for cognate interactions (simulating the selectivity filter shown to work in trans). Once formed, the complexes were not dissociated. The number of trans complexes of the endosomal Q-SNAREs with synaptobrevin or VAMP4 were calculated under four different conditions: (I) both exocytic and endosomal SNAREs are randomly distributed, (II) the concentration of the exocytic SNAREs at the contact site is reduced by a factor of 10, (III) the concentration of early endosomal SNAREs is increased three-fold at the contact site and (IV) the reduction in exocytic SNAREs is introduced simultaneously with the increase in endosomal SNAREs. The proportion of cognate versus non-cognate complexes increases steadily from model I to IV (Figure 3.7). If we assume that four complexes are required for the fusion event, it is clear from the simulation that specificity alone (model I) is not sufficient for fusion, but only its co-operative function with a mild enrichment/de-enrichment of the appropriate SNAREs results in fusion on every run (model IV). It can be therefore suggested that the coupled function of the two mechanisms is what promotes selectivity in SNARE pairing and further ensures the specificity of intracellular fusion processes.

The results presented so far indicate that despite the promiscuity in associations and localization between non-cognate SNAREs in cis, there are regulatory mechanisms that ensure in a co-operative fashion the specificity of SNARE pairing for fusion. The function of a specificity filter allows only cognate SNAREs to associate in trans. The spatial segregation of cognate SNAREs in the fusion sites, in combination with the lateral exclusion of non-cognate isoforms, provides a second level of control. Nevertheless, the actual molecular players mediating the SNARE function specificity are not yet fully characterized and the cellular conditions that allow this highly regulated machinery to perform are unknown. What is the response of endosomal fusion to cellular stress conditions and is specificity in SNARE function sacrificed when the fusion machinery is challenged? To address 
these issues, I modulated the endosomal fusion machinery by downregulating the expression of individual cognate and non-cognate SNAREs and I studied the status of endosomal fusion and trafficking at this cellular background.

\subsection{Endosomal function upon SNARE knock-down}

As shown in the previous section, early endosomal fusion is a highly specialized and regulated process. Despite the great variety of SNAREs present on the endosomal membrane, homotypic endosomal fusion is mediated specifically by the early endosomal SNAREs, since the combinatorial function of intrinsic membrane mechanisms ensure that only the cognate SNARE partners engage in complex for fusion.

Nevertheless, the properties and function of early endosomal SNAREs, as well as their involvement in homotypic endosomal fusion have been characterized only by following in vitro approaches. Use of antibodies or recombinant SNARE fragments in assays of fusion reconstitution in vitro were the only means suggesting a role for the syntaxins 13 and 6, vtila and VAMP4 in endosomal fusion (Brandhorst et al., 2006; Mills et al., 2001; Prekeris et al., 1998). Therefore, the exact mode of function of these molecules within the cellular context remains unclear.

Therefore, I decided to downregulate the expression of these molecules in PC12 cells and to monitor the cellular response to this perturbation. The elimination (knock-out) or downregulation (knock-down) of the expression of a given protein can provide valuable information about its intracellular role and its importance for cell homeostasis and viability. More specifically for SNAREs, exocytosis was impaired in neurons when synaptobrevin (Schoch et al., 2001) or SNAP-25 (Washbourne et al., 2002) were knocked-out, indicating the role of these proteins in the fusion of synaptic vesicles. In addition, by use of knock-down approaches, new functions were assigned to hitherto uncharacterized SNAREs and new roles to already known ones. For example, syntaxin 6 was shown to play an additional role in the transport of caveolae to the plasma membrane (Choudhury et al., 2006), while the exocytic SNARE SNAP-25 was implicated in endocytic recycling(Aikawa et al., 2006).

While the findings summarized above show that individual SNAREs are indeed specialized for one or several related fusion reactions, it has become clear 
that the SNARE system is more flexible than originally envisioned. For many SNAREs, knock-out or knock-down has little impact on cell function, with no measurable changes being observable in the intracellular pathways in which the targeted SNAREs are known to play a role (see also Discussion). For example, knock-down of SNAP-23 had no effect on the constitutive exocytosis of alkaline phosphatase, even though the protein had been shown to function in constitutive secretion (Okayama et al., 2007). Downregulation of syntaxins 6 and 16 did not affect transferrin uptake and recycling (Amessou et al., 2007; Choudhury et al., 2006). Along the same lines, knock-out of the late endosomal SNARE vti1b had neither an effect on the animals' fertility or viability, nor on late endosomal and lysosomal function (Atlashkin et al., 2003), while syntaxin $1 \mathrm{~A} \mathrm{KO}$ mice were fertile and viable (Fujiwara et al., 2006).

How can the fusion machinery tolerate so robustly the loss or downregulation of some of its main functional components? What are the mechanisms behind the observed adaptation of the cellular machinery to SNARE loss? Two scenarios that may complement each other are possible. First, SNAREs of the same subfamily may substitute for each other, thus providing functional redundancy and explaining the lack of phenotype in knock-out approaches. For instance, some SNAREs such as syntaxin 1, synaptobrevin or other SNAREs belonging in the same subfamily can functionally replace each other to a varying degree, as previously shown for synaptobrevin and cellubrevin (Borisovska et al., 2005) or SNAP-25 and SNAP23 (Delgado-Martnez et al., 2007). Second, SNAREs are expressed at high levels, which may drastically surpass the cellular needs, suggesting a model of abundance as compensation. Thus, even a more than $90 \%$ reduction (as is reached in many RNA interference (RNAi) experiments) may not suffice to perturb function. For example, neuronal exocytic SNAREs are expressed at hugely abundant levels (Takamori et al., 2006; Walch-Solimena et al., 1995), with fusion persisting even when the free SNARE pool is substantially depleted (Kawasaki et al., 1998).

To get a deeper insight into the intracellular role of early endosomal SNARES, I downregulated their expression by RNAi means and monitored the effect of this perturbation on endosomal fusion and function, as well as on cellular homeostasis and viability. Several properties of early endosomal SNAREs, such as their relative 
amounts on the endosomal membrane, their promiscuous association with exocytic SNAREs in cis as well as the mechanisms of their specific pairing in trans, have been well characterized. Therefore, these proteins also served as an ideal experimental tool for studying the degree of robustness exhibited by the fusion machinery under low availability of its components.

\subsubsection{Knock-down of early endosomal and exocytic SNAREs}

RNA interference is a broadly-used gene regulatory mechanism that limits the mRNA level of a targeted gene by activating a sequence-specific RNA degradation process. The silencing pathway is initiated by the introduction of long doublestranded RNAs (dsRNAs: typically > 200 nucleotides (nt)) in the organism or cell type of choice. The dsRNAs are then processed into 20-25 nt small interfering RNAs (siRNAs) by an RNase III-like enzyme called Dicer (initiation step). The siRNAs assemble into endoribonuclease-containing complexes known as RNA-induced silencing complexes (RISCs), which unwind in the process. The siRNA strands subsequently guide the RISCs to find complementary RNA molecules, where they cleave and therefore destroy the cognate RNA (effecter step). For experimental use of this endogenous pathway in mammalian cells, the introduction or expression of a duplex of short siRNAs, complementary to a part of the RNA corresponding to the targeted gene, is enough to trigger the cascade, initiate destruction of the transcript and prevent protein expression.

\section{Transient knock-down}

For the knock-down of SNAREs in PC12 cells, I initially followed the approach of transiently silencing these proteins by introducing small, synthetically-produced, siRNA duplexes in the cells, using conventional transfection means. The efficiency of silencing for a given siRNA-duplex depends on its length, secondary structure, sugar backbone and sequence specificity. Many laboratories have developed algorithms that can predict the optimal sequences along a given RNA molecule to be used as siRNAs. For the design of siRNA duplexes against the early endosomal SNAREs (syntaxins 13, 6 and 16, vtila and VAMP4), as well as the exocytic SNARE synap- 

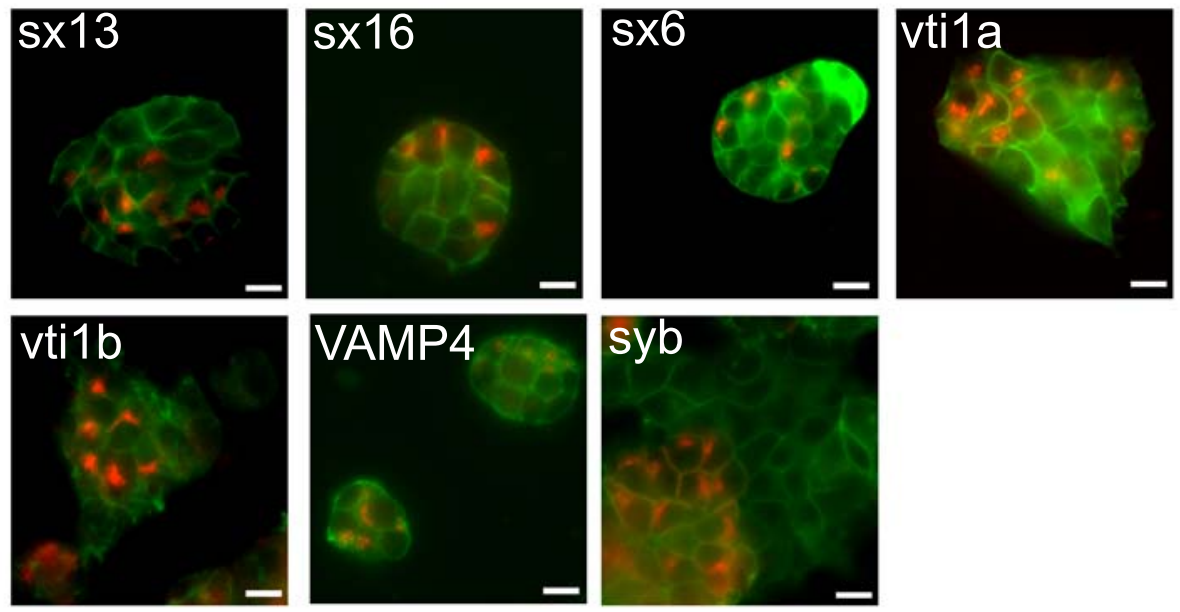

Figure 3.8: Transient knock-down of SNAREs in PC12 cells. PC12 cells were transfected with siRNA oligonucleotides against syntaxin 13, 16, 6, vti1a, vti1b, VAMP4 and synaptobrevin, respectively. $72 \mathrm{~h}$ post-transfection, cells were fixed and immunostained against the membrane protein SNAP-25 (green) and the respective siRNA-targeted protein (red). Note the absence of the red signal from the knock-down cells. Scale bar: $10 \mu \mathrm{m}$

tobrevin, and the late endosomal SNARE vti1b in PC12 cells, I retrieved the cDNA sequence for each protein of interest from the Rat Genome Database and introduced it in the BLOCK-iT ${ }^{\mathrm{TM}}$ RNAi Designer program, provided by Invitrogen. This algorithm, taking into consideration all the parameters determining siRNA-efficiency, suggests ten sequences (sense and anti-sense) of 21 nucleotides in length that are suspected to robustly silence the expression of the targeted RNA. Three sequences per protein of interest were tested and their silencing potency was evaluated.

PC12 cells were transfected with individual siRNA oligonucleotides against the proteins of choice and the efficiency of the knock-down was addressed by immunostaining, 72 hours post-transfection. As shown in Figure 3.8, the transient silencing of all SNAREs tested was possible. The morphology and viability of the knockeddown cells did not seem to be affected. Quantification revealed that $40 \%$ of the cells exhibited significantly reduced expression levels of the targeted SNARE protein. The levels of vtila expression from a population of cells transfected with an siRNA against vtila was also calculated using immunoblotting and revealed that vtila expression was downregulated by about $40 \%$. Taking these data together, it can be 
concluded that in each transfected cell, only minor residual levels of the targeted protein were left, implying the high silencing efficiency of this approach.

\section{Generation of stably-silenced PC12 cell lines}

The use of siRNA-oligonucleotides against early endosomal SNAREs and synaptobrevin in PC12 cells showed that SNARE silencing is possible in this cell type, with a high efficiency per transfected cell but with a rather small and variable population of cells being knocked-down per transfection experiment. Since these experimental limitations did not allow biochemical studies on the knock-down cells, I proceeded with the generation of PC12 cell lines that would be stably downregulated for three early endosomal Q-SNAREs (syntaxin 13, 6 and vti1a) and for the exocytic synaptobrevin, respectively. These cells were a well-characterized, homogeneous population that could be proliferated to high amounts and for prolonged periods. The choice of targeting the expression of the three endosomal Q-SNAREs was made due to the established role of these proteins in early endosomal fusion. Syntaxin 13, initially named as syntaxin 12, exhibits a clear localization on early and recycling endosomes (Prekeris et al., 1998; Tang et al., 1998) and perturbation of its function either by addition of specific anti-syntaxin 13 antibodies or by use of recombinant, cytosolic syntaxin 13, interfered with endosomal fusion and trafficking (Brandhorst et al., 2006; McBride et al., 1999; Prekeris et al., 1998). Similarly, syntaxin 6 and vtila have been implicated in early endosomal fusion (Brandhorst et al., 2006; Mills et al., 2001), even though their intracellular localization is not strictly endosomal and the involvement, especially of syntaxin 6 , in other intracellular fusion processes has been suggested (Wendler and Tooze, 2001). VAMP4 was excluded at this point from the study due to the limited characterization concerning the early endosomal role of this SNARE. However, I decided to downregulate the expression of the exocytic R-SNARE synaptobrevin, even though it does not participate in endosomal fusion, as shown in Figure 3.1. Nevertheless, the protein is highly abundant at the early endosomal compartment (Table 3.1) and it is engaged in strong and numerous interactions with the endosomal Q-SNAREs (see Introduction, Figure 1.4). Since mass action is thought to be the reason for these associations, downregulation of synap- 
tobrevin levels might decrease the amount of non-cognate interactions and affect the efficiency or regulation of endosomal fusion. Since in vitro assays are valuable for the direct study of organelle fusion but require a source of cells that are stablydownregulated, a PC12 cell line stably knocked-down for synaptobrevin was also created.

SiRNAs in mammalian cells can be expressed as fold-back stem-loop structures that give rise to siRNAs with a small loop, called small hairpin siRNAS (shRNAs). These molecules can then be incorporated to RISC-complexes and follow the siRNA silencing pathway. CDNAs encoding for such shRNA structures were designed for each target SNARE, using the algorithm provided by the siRNA Target Designer (Promega). In parallel, a control sequence was also prepared. The cDNA designed to target synaptobrevin expression was scrambled and the new sequence, having the same GC content as the other sequences but no complementary sequence in the rat genome, was used as a control for changes caused only by the shRNA expression itself and not by its specific action. Cells stably transfected with this construct were named scrambled. The sequences were subsequently cloned into the psiSTRIKE U6 Hairpin Neomycin vector (Promega), which expresses short hairpin RNAs (shRNAs) under a U6 RNA polymerase III promoter and which additionally allows the selection of transfected cells in presence of the antibiotic G418. PC12 cells of early passage were transfected by electroporation with each of the generated shRNA-vectors, which were previously linearized by digestion with BsaI. Cells from one electroporation cuvette were plated in high dilution in 15 -cm plates in medium containing $500 \mu \mathrm{g} / \mathrm{ml}$ G418. After 10 days in selection medium, the surviving cells were transfered to a $15 \mathrm{~mm}$-diameter well and were passaged to bigger plates, every time they reached confluency. After approximately 1 month of propagation, cells were viable, exhibited normal morphology and were used in experiments or frozen for storage.

The success of the knock-down was checked by immunostaining and immunoblotting. As shown in Figure 3.9, the SNARE silencing seemed to be rather homogeneous and efficient as the immunostaining signal was almost completely abolished compared to wild type cells for each of the four targeted proteins. In parallel, 

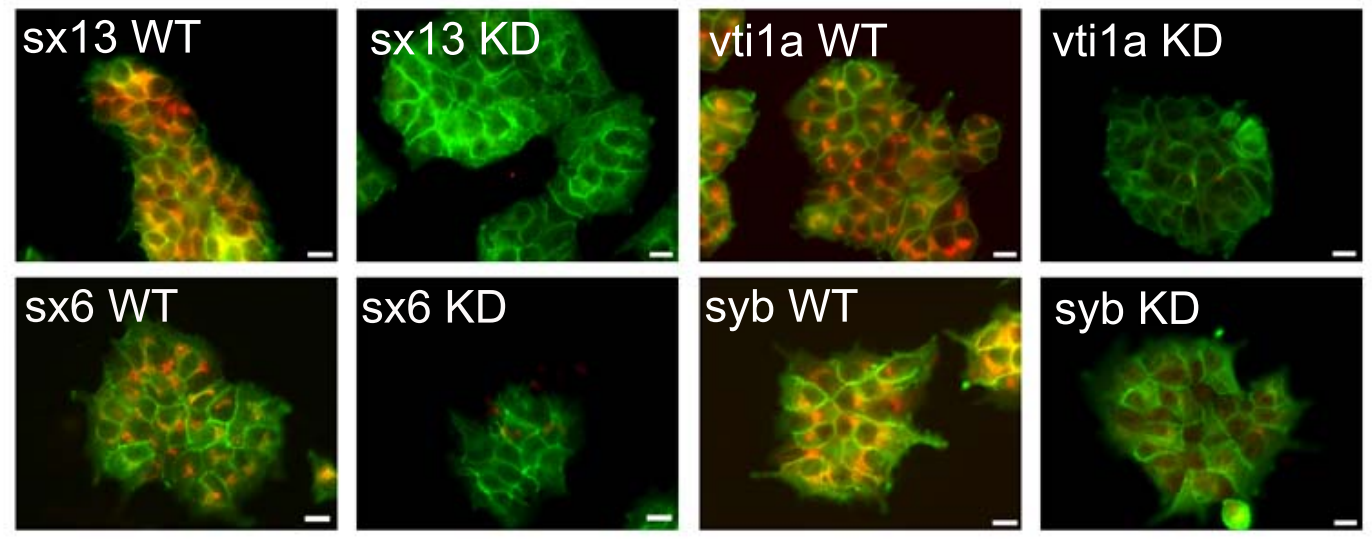

Figure 3.9: The expression of individual SNAREs is efficiently downregulated in the knock-down cell lines. Wild type PC12 cells (WT) and cells stably expressing an shRNA against syntaxin 13, vti1a, syntaxin 6 and synaptobrevin, respectively (KD), were fixed and immunostained against the membrane protein, SNAP-25 (green) and against the respective shRNA-targeted protein (red). Note the absence of the red signal from the knock-down cells (the faint signal persisting in the synaptobrevin KD cells corresponds to artifactual nuclear immunostaining). Scale bar: 10 $\mu \mathrm{m}$.

homogenates from wild type, control transfected and knocked-down cells were analyzed by SDS-PAGE and analyzed by immunoblotting for the targeted proteins. Actin was used as a loading control. Quantification of immunoblots from multiple independent experiments revealed a $90 \%$ reduction in the expression of syntaxin 13 , vtila and synaptobrevin, respectively and a $60 \%$ downregulation in the expression of syntaxin 6 (Figure 3.10).

The good viability and the normal proliferation rates of the knocked-down cells provided some initial indications of compensation mechanisms that leave the cells unaffected by the silencing of individual SNAREs. Could changes in the expression levels of other SNARE molecules function as such a mechanism? It is conceivable that the upregulation of a related SNARE molecule could provide the fusion machinery with the necessary SNARE molecules to compensate for the downregulation of the targeted SNARE partners. I therefore checked in each cell line for the expression levels of SNAREs that can be found on the early endosomal membrane, such 


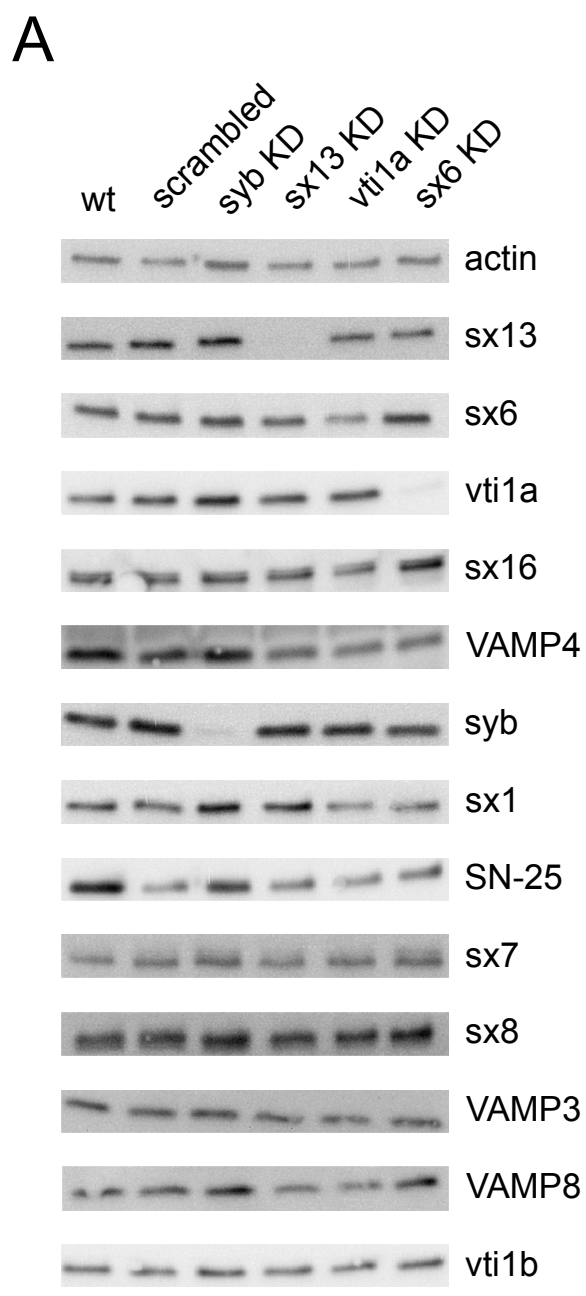

B

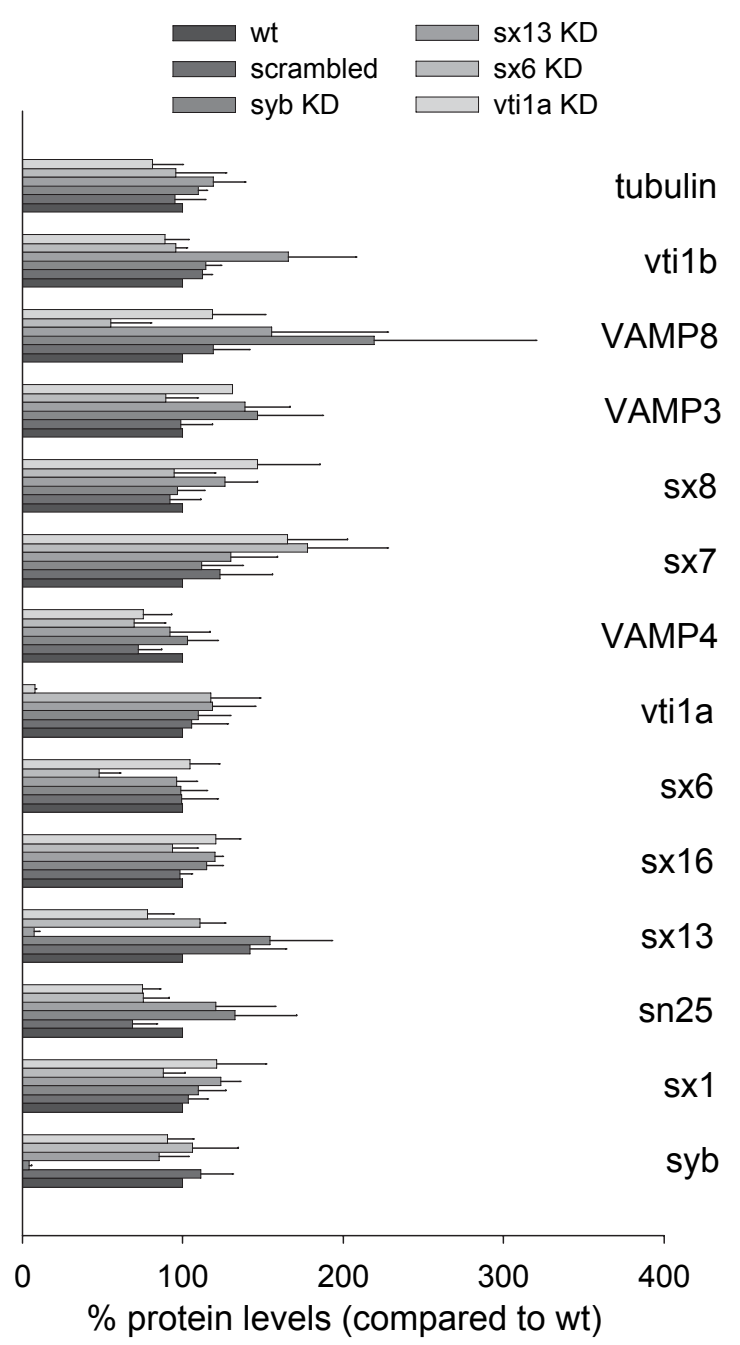

Figure 3.10: The expression of other SNAREs does not change in the stably knockeddown cells. A) Homogenates from wild type PC12 cells, scrambled and knock-down cells were analyzed for the expression of several proteins by immunoblot. Equal amounts of protein were loaded per lane. The levels of the shRNA-targeted proteins were severely reduced, but there were no significant changes in the expression of other SNAREs or cytoskeleton markers. B) The amount of protein for each cell line was normalized to the actin amount (loading control), and then presented as percentage of the amount in wild type cells, which was set to $100 \%$. Averages \pm s.e.m. from at least three independent experiments are shown.

as the early endosomal, exocytic and late endosomal SNAREs. Even though the individual blots exhibited some degree of variability, the quantification of multiple experiments (Figure 3.10B) showed that none of the tested proteins exhibited any 
significant change in its expression levels. These results document that the RNAi approach was highly specific for the targeted proteins and also reduce the possibility of compensation for the SNARE loss via upregulation of a related SNARE.

\subsubsection{Endosomal function is not affected in the knock-down cells}

Irrespective from its internalization mode, recently endocytosed material is typically first transferred to the early endosomal compartment, where its sorting takes place. From there, it can be targeted back to the plasma membrane, to the lysosome for degradation, or even to the Golgi apparatus. Since this delivery of material through the early endosomes requires the fusion of these organelles with each other but also with a variety of other compartments, I next checked whether the silencing of individual SNAREs has any impact on endocytosis, sorting and trafficking in PC12 cells. For this purpose, I chose fluorescently-labeled markers that follow different internalization and trafficking pathways and compared their intracellular targeting between wild type and knock-down cells, using fluorescence microscopy.

In most mammalian cell types and under normal conditions, the uptake of receptor-bound ligands and extracellular fluid results mainly from the formation of clathrin-coated vesicles $(\mathrm{CCVs})$. Two commonly used markers of clathrindependent, receptor-mediated endocytosis that subsequently follow different trafficking routes are the iron-laden transferrin ( Tfn) that binds to the Tfn receptor and the cholesterol-laden low-density lipoprotein (LDL) particles that bind to the LDL receptor. Upon internalization, both markers can be found in the early endosomal compartment, where their sorting takes place. After losing its bound iron, transferrin remains bound to its receptor and rapidly recycles back to the plasma membrane, while LDL dissociates from its receptor and is targeted to late endosomes/lysosomes for degradation. To check for the impact of SNARE silencing on the trafficking of these molecules, I allowed wild type, scrambled and knocked-down cells to internalize fluorescently-labeled transferrin or LDL for $5 \mathrm{~min}$ at $37^{\circ} \mathrm{C}$, fixed the cells and acquired images, using an epifluorescence microscope. Quantification of the internalized amount of transferrin and LDL for each cell type revealed no striking differences between wild type and knocked-down cells (Figure 3.11, panels A and C). 
In addition, transferrin recycling was studied by allowing the cells to internalize labeled transferrin for $5 \mathrm{~min}$ and further incubating them for $10 \mathrm{~min}$ in transferrin-free medium at $37^{\circ} \mathrm{C}$. I then quantified the remaining intracellular fluorescent signal and found that knocked-down cells recycled transferrin as efficiently as wild-type ones (Figure 3.11B). Similar observations were made when transferrin trafficking was studied in PC12 cells transiently transfected with siRNA oligonucleotides targeting early endosomal SNAREs. 72 hours post-transfection, I allowed cells to internalize labeled-transferrin, and I either fixed or chased them for additional 10 min before fixation. I then performed immunostainings against the respective siRNA-targeted proteins to identify the knocked-down cells and compared the fluorescent signal corresponding to transferrin that was contained in untransfected and SNARE-silenced cells. In all cases, transferrin uptake and recycling was not affected by SNARE loss (Figure 3.12).

In parallel to the regulated, receptor-mediated endocytosis, fluid-phase uptake is also a vital endocytic process and can be easily measured by the intracellular accumulation of labeled compounds present in the medium. The degree of internalization of fluid-phase markers is directly proportional to their concentration in the medium and the volume encased by the transport vesicles. To study this entry pathway, I checked for the internalization properties of two differentially-sized markers, the relatively large, $10 \mathrm{kDa}$-dextran and the small, styryl, FM-dye AM2-10 in wild type and knocked-down cells. The efficient uptake of dextran from the stably knocked-down cells (quantified in Figure 3.11) and of AM2-10 from the transientlysilenced cells (Figure 3.12) showed that SNARE downregulation does not seem to affect fluid-phase endocytosis in PC12 cells.

Many intracellular trafficking pathways have been unraveled by the study of infectious bacterial toxins that 'hijack' the trafficking machinery to reach their compartment of action. Such an example is the Cholera toxin (CT) produced by Vibrio cholerae. Cholera toxin enters the cell by binding the ganglioside GM1 at the cell surface via its B-subunit and associating with lipid rafts. The toxin's internalization can occur via clathrin- and caveolae-dependent or independent mechanisms and the toxin, passing through the endosomal compartment, is targeted to the Golgi ap- 
A

Tfn uptake

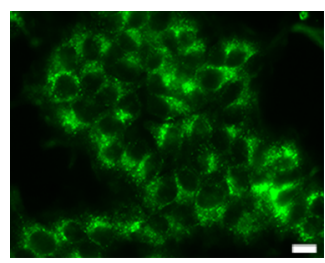

C

LDL uptake

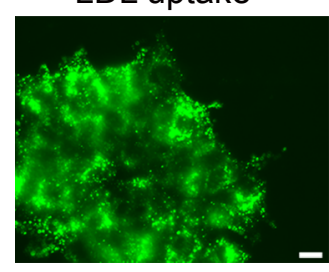

$E$

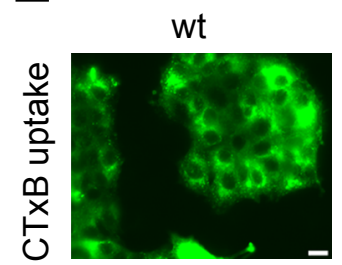

B Tfn recycling

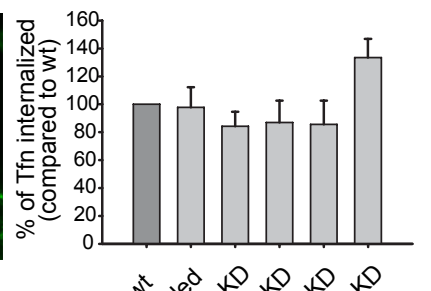

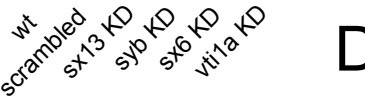

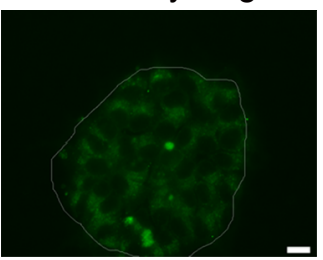

D

\section{Dextran uptake}

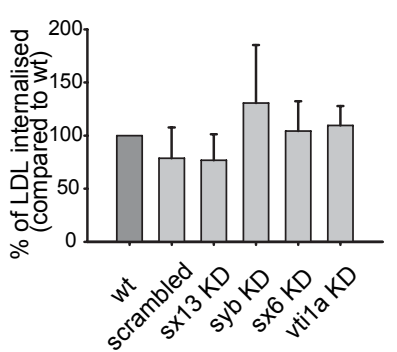

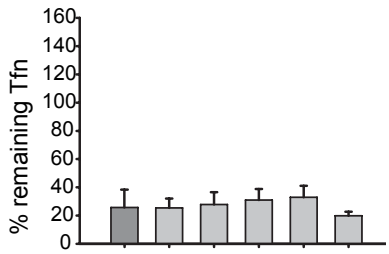

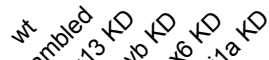

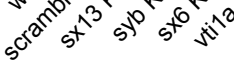

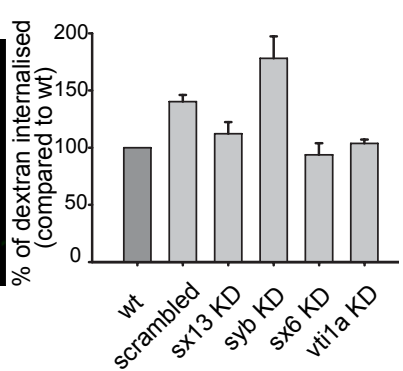
sx13 KD

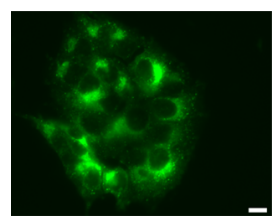

$\mathrm{sx6} \mathrm{KD}$

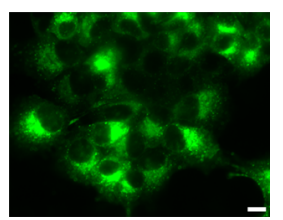

syb KD

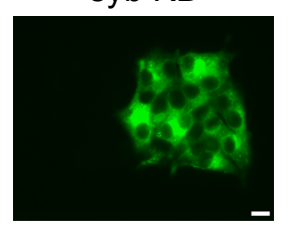

vti1a KD

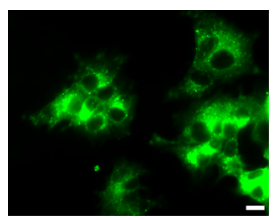

Figure 3.11: Transferrin, LDL and Dextran trafficking is not affected in knocked-down cells. A) Wild type PC12, scrambled or knocked-down cells were incubated for 5 min at $37^{\circ} \mathrm{C}$ with Alexa 594-transferrin. Cells were fixed and imaged. A typical example of the transferrin uptake by syntaxin $13 \mathrm{KD}$ cells is shown (left panel, transferrin in green). The average fluorescent signal was calculated for each cell line and expressed as percentage of the value corresponding to the wt cells (set to 100\%). Means \pm s.e.m. from three individual experiments are presented (right panel). B) The different cells lines were incubated with Alexa 594-transferrin for $5 \mathrm{~min}$ at $37^{\circ} \mathrm{C}$. Cells were washed and incubated for additional $10 \mathrm{~min}$ in transferrin-free medium to study transferrin recycling. A typical image for syntaxin $13 \mathrm{KD}$ cells is shown (left panel, transferrin in green). Notice that little transferrin remains intracellular. The average fluorescent signal was measured for each cell line and expressed as percentage of the internalized transferrin for the respective cell line (means \pm range of values from two individual experiments are presented, right panel). C-D) DiI-LDL (C) or Alexa 594-Dextran $10 \mathrm{kDa}(\mathrm{D})$ were internalized for 5 min at $37^{\circ} \mathrm{C}$ from the various cell lines. Typical images for syntaxin $13 \mathrm{KD}$ cells are shown (left panel of $\mathrm{C} / \mathrm{D}$ ). Average fluorescence for each cell line is presented as percentage of the fluorescence of wt cells (set to $100 \%$ ). Means \pm s.e.m. from three individual experiments are shown (right panel of C/D). E) Alexa 594-Cholera toxin B subunit $(\mathrm{CTxB})$ was applied for $30 \mathrm{~min}$ at $4^{\circ} \mathrm{C}$. Unbound $\mathrm{CTxB}$ was washed out and cells were transferred to $37^{\circ} \mathrm{C}$ for $40 \mathrm{~min}$. Cells were fixed and imaged. Notice that CTxB (green) was successfully internalized by all cell lines. All scale bars: $10 \mu \mathrm{m}$. 


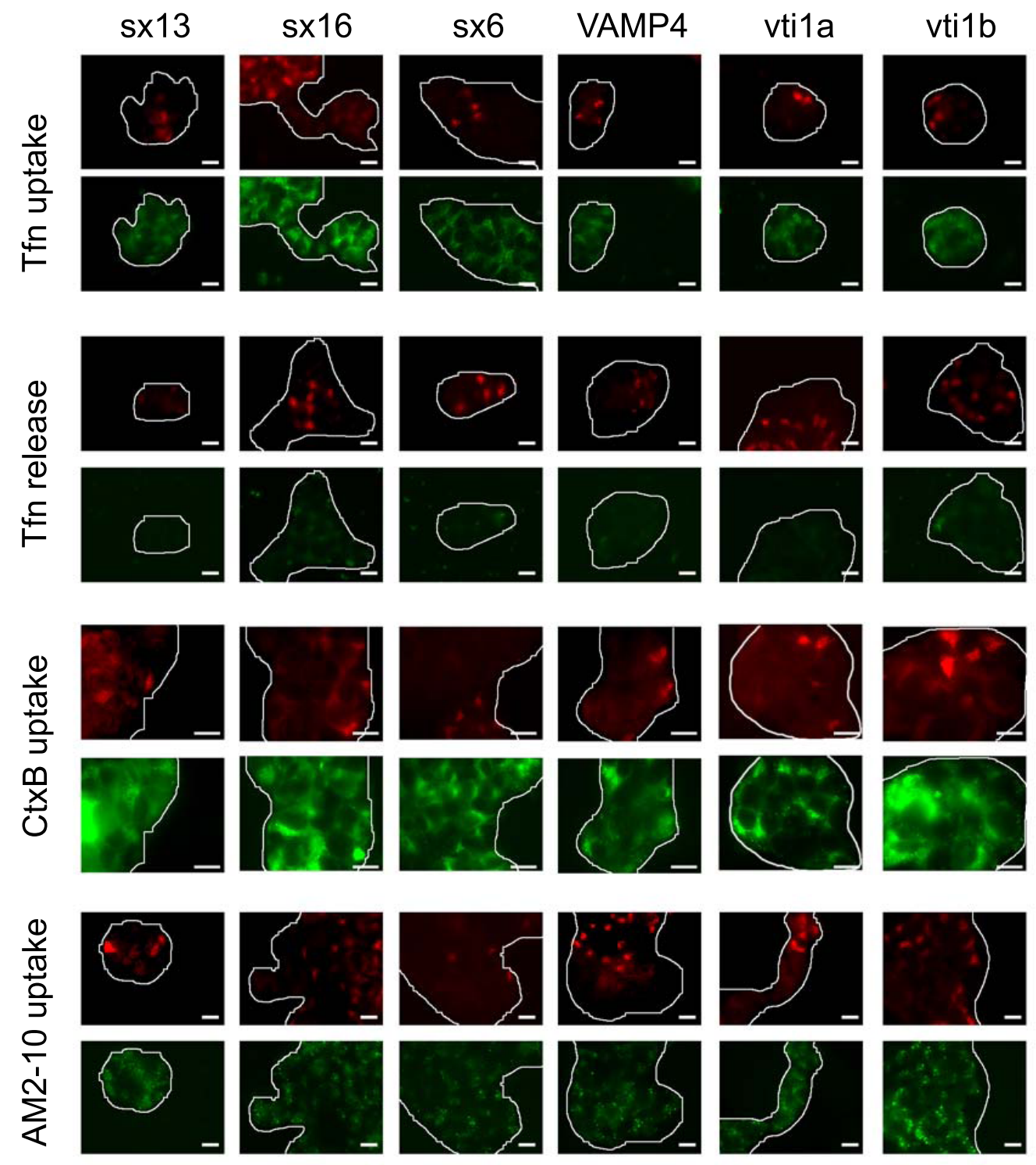

Figure 3.12: Various endocytic pathways are not affected by transient knock-down of early endosomal SNAREs. PC12 cells were transfected with siRNA oligonucleotides against syntaxin 13, 16, 6, vti1a, vti1b and VAMP4, respectively. 72 hours post transfection, cells were incubated with fluorescently-labeled marker (see below), then fixed and immunostained against the respective siRNA-targeted protein. Typical images are shown, green: internalized marker, red: SNARE protein. Tfn uptake: Cells were incubated for $5 \mathrm{~min}$ at $37^{\circ} \mathrm{C}$, with Alexa 594labeled transferrin. Tfn release: Cells were incubated with Alexa 594-labeled transferrin for 5 min at $37^{\circ} \mathrm{C}$ and then chased for $10 \mathrm{~min}$ in transferrin-free medium to study transferrin recycling. CTxB uptake: Alexa 594-labeled Cholera toxin B subunit (CTxB) was applied for 30 min at $4^{\circ} \mathrm{C}$. Unbound CTxB was washed out and cells were transferred to $37^{\circ} \mathrm{C}$ for 40 min. AM2-10 uptake: Cells were incubated for 5 min with $\mathrm{AM} 2-10$ at $37^{\circ} \mathrm{C}$, to study the endocytosis of small vesicles. Scale bar: $10 \mu \mathrm{m}$. 


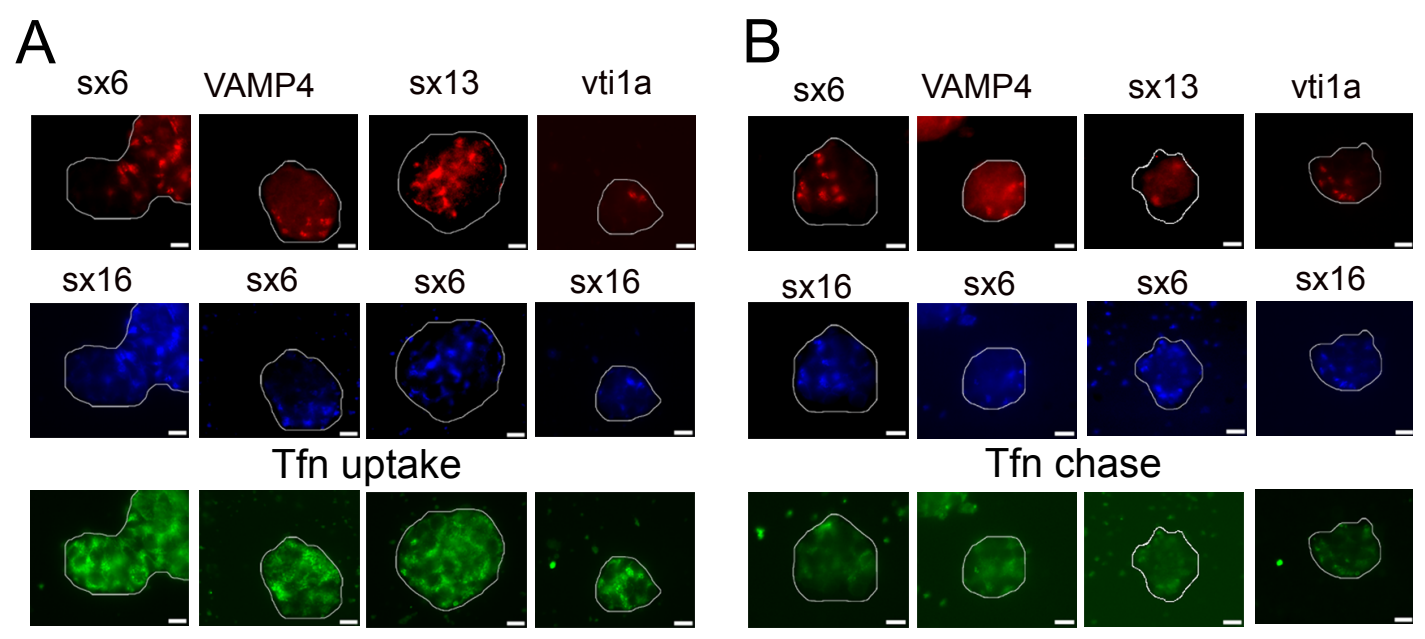

Figure 3.13: Transferrin trafficking is not affected by the simultaneous knock-down of two SNAREs. PC12 cells were transfected with a pair of siRNA oligos targeting two different SNARE molecules. $72 \mathrm{~h}$ post transfection, cells were incubated with Alexa 594-labeled transferrin for $5 \mathrm{~min}$ and they were either fixed (A) or further chased for $10 \mathrm{~min}$ in transferrin-free medium before fixation (B). Cells were subsequently stained for the SNAREs targeted by the siRNAs used in each experiment. Note that transferrin uptake and recycling are not affected by the simultaneous downregulation of two SNAREs. Scale bar: $10 \mu \mathrm{m}$.

paratus or the ER. Using labeled Cholera toxin B subunit (CTxB) and following its trafficking for $40 \mathrm{~min}$ after induction of endocytosis, I showed that cells stably or transiently knocked-down for individual SNAREs exhibit normal Cholera toxin uptake and trafficking (Figures 3.11, 3.12).

The data presented so far indicate that downregulation of individual SNAREs has no severe impact on endosomal trafficking and clearly suggest that the endosomal fusion machinery can successfully compensate for a major loss of individual components. What are the limits of this adaptation mechanism and how does the cell respond when several SNARE molecules are knocked-down in parallel? To address this question, I transfected PC12 cells simultaneously with two siRNA oligonucleotides, targeting two different SNARE molecules (syntaxin 6 and syntaxin 13; syntaxin 6 and syntaxin 16; syntaxin 6 and VAMP4; syntaxin 16 and vti1a). Three-days post-transfection, I allowed the cells to internalize and/or recycle labeled transferrin, immunostained them against the siRNA-targeted proteins, and imaged them. 


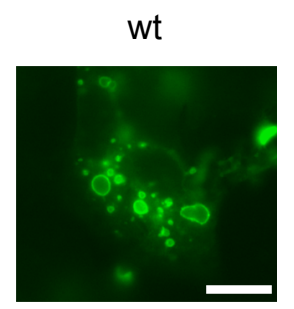

sx13 KD

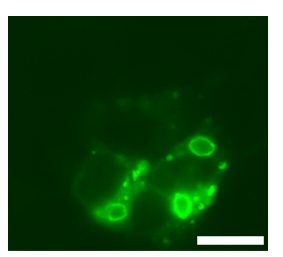

$\mathrm{sx6} \mathrm{KD}$

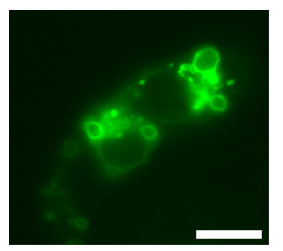

syb KD

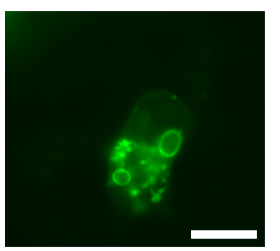

vti1a KD

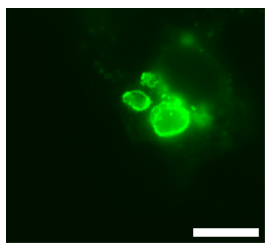

Figure 3.14: Endosomal fusion functions in stably knocked-down cells. Wild type PC12 cells and PC12 cells stably knocked-down for syntaxin 13, syntaxin 6, vti1a and synaptobrevin, respectively, were transfected with GFP-Rab5 Q79L. Two days post-transfection, cells were fixed and visualized for the presence of enlarged GFP-Rab5 positive endosomes. Scale bar: $10 \mu \mathrm{m}$.

As shown in Figure 3.13, double transfected cells were still able to internalize and recycle transferrin, suggesting the existence of a robust compensation mechanism that can deal with the absence of more than one SNARE isoform.

Since the main function of the endosomal compartment, trafficking, was not affected by the SNARE downregulation, I also tested whether endosomal fusion itself is still functioning in the knocked-down cells or whether alternative pathways are taking over the sorting function. I transfected wild type and knocked-down cells with the GFP-tagged constantly-active Rab5 mutant Q79L (see Figure 3.2) and checked for the generation of large endosomes, indicative of enhanced homotypic fusion. Two days post-transfection, GFP-positive, enlarged organelles were present in all cell lines, suggesting that early endosomal fusion has remained functional (Figure $3.14)$.

\subsubsection{NGF-differentiation and exocytosis remain unaffected upon SNARE downregulation}

Early endosomes are not only involved in intracellular sorting, but also play a role in membrane transfer. Addition of new membrane at the end of growing neurites, for example, is mediated by fusion of intracellular vesicles with the plasma membrane and the early endosomal compartment serves as a membrane source for this process. PC12 cells have the ability to differentiate and exhibit neurite outgrowth upon 
exposure to NGF (Greene and Tischler, 1976). I grew wild type and knockeddown cells in medium containing $100 \mathrm{ng} / \mathrm{ml} \mathrm{NGF}$ for 1,2 or 3 days and counted the length of the longest neurite per cell after the different time intervals. Averaging the measurements from many cells from each cell line, I showed that NGF-differentiation is insensitive to downregulation of any of the targeted SNAREs (Figure 3.15A), further supporting the functional integrity of the early endosomal compartment in knocked-down cells.

To complete the characterization of the knocked-down cells lines, I finally studied catecholamine release. PC12 cells contain dense core vesicles (DCVs), which are packed with catecholamines and undergo exocytosis upon increase of intracellular $\mathrm{Ca}^{2+}$ levels. I incubated wild type and knocked-down cells in medium containing ${ }^{3} \mathrm{H}$-norepinephrine overnight, in order to load the DCVs. I stimulated the cells with high $\mathrm{K}^{+}$application for $15 \mathrm{~min}$ and measured how much norepinephrine was released compared to the amount initially internalized. In agreement with studies on chromaffin cells (Borisovska et al., 2005), the downregulation of synaptobrevin, a protein known to function in fusion with the plasma membrane, did not cause any effect in the ability of these cells to release the loaded norepinephrine (Figure $3.15 \mathrm{~B})$.

\subsubsection{SNARE silencing does not affect early endosomal fusion in vitro}

I previously showed that endosomal fusion is still possible in knocked-down cells as enlarged endosomes could be formed upon transfection of the Rab5 mutant. Nevertheless, possible alterations in the properties of the fusion machinery due to SNARE silencing may not be detectable by this read-out. The most convenient way to directly (and quantitatively) investigate endosome fusion is by performing in vitro assays (Brandhorst et al., 2006; Gruenberg and Howell, 1989). Applying the microscopic fusion assay described in 2.2.7, I incubated wild type and knockeddown cells with Alexa 594 (red) or Alexa 488 (green) - labeled dextran for 5 min, to specifically mark early endosomes. Then, I homogenized the cells and isolated post-nuclear supernatants (PNS) containing the labeled organelles. Fusion reactions were prepared by mixing the differentially labeled PNSs with cytosol and an ATP- 
A

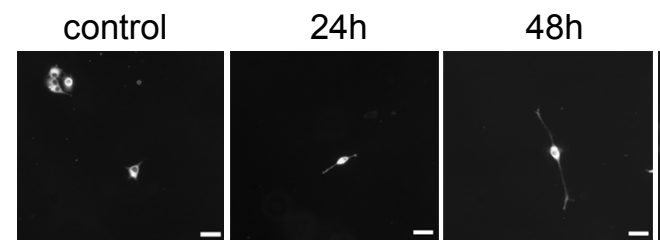

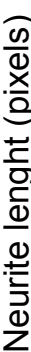

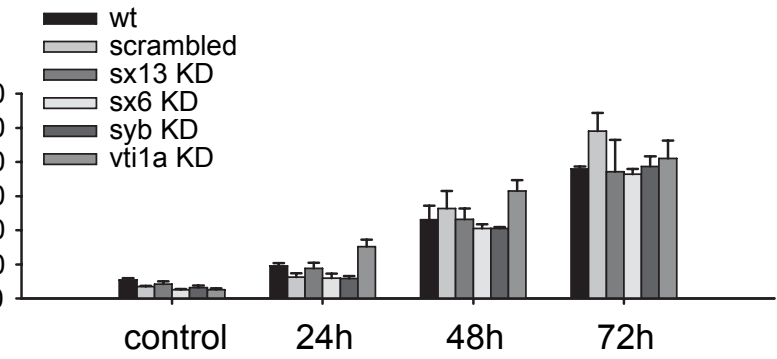

B $72 \mathrm{~h}$
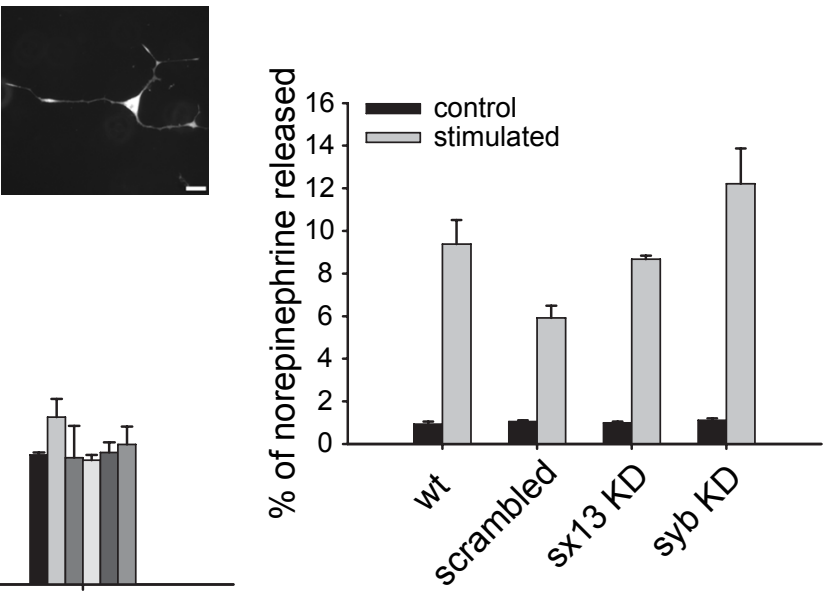

Figure 3.15: NGF differentiation and norepinephrine release are not altered upon SNARE downregulation. A) Cells were grown in presence of $100 \mathrm{ng} / \mathrm{ml}$ NGF for 24, 48 and 72 h. In control cells, no NGF was applied. Cells were fixed after the respective period and were visualized in solution containing AM2-10, which stains all membranes. Typical pictures are presented from syntaxin $6 \mathrm{KD}$ cells. Scale bar: $34 \mu \mathrm{m}$. The length of the longest neurite was measured for 10-20 cells from each cell line and for each time interval. Means \pm s.e.m. are shown from three individual experiments. B) Wild type PC12 cells and PC12 cells stably expressing a control transfected shRNA or a shRNA targeting the expression of syntaxin 13 or synaptobrevin were incubated overnight in medium containing ${ }^{3} \mathrm{H}$-norepinephrine. Next day, cells were incubated in Krebs solution (control) or in Krebs solution containing $90 \mathrm{mM} \mathrm{KCl}$ (stimulated) for 5 min at $37^{\circ} \mathrm{C}$. Supernatants were collected, cells were lysed in Triton-containing buffer and the radioactive content of these two samples was measured for each cell line. The amount of norepinephrine released was calculated by dividing the norepinephrine present in the supernatant by the sum of the norepinephrine present in supernatant and cell lysate. Means \pm s.e.m. from three individual experiments are shown.

regenerating system and these were then incubated for $45 \mathrm{~min}$ at $37^{\circ} \mathrm{C}$. Fusion was subsequently quantified by determining the fraction of 'green' organelles colocalizing with 'red' organelles in fluorescence images, as described in section 2.2.7 and shown in panel A of Figure 3.16. Reactions were also performed in presence of an ATPdepleting system, to check for the dependence of the fusion process on ATP. None of 
the knock-downs eliminated ATP-dependent fusion; moreover, PNS fractions from different knock-down lines fused together efficiently, with the amount of fusion being well within wild type levels (Figure 3.16B).

Since the protocol described above provides information only about the maximum amount of fusion, I also asked whether the rate of endosomal fusion is altered upon SNARE silencing. I tested the kinetics of fusion for control and knock-down organelles by arresting the reaction at various time points, between 0 and $45 \mathrm{~min}$, and checking for the amount of fusion completed until the respective time point. Comparing the time needed for the reaction to reach $50 \%$ of its maximum for each of the tested cell lines, I showed that endosomal fusion exhibits similar kinetics in all cases, with no apparent sensitivity to syntaxin 13 or synaptobrevin downregulation (Figure 3.16C).

To directly address the issue of compensation by recruitment of new SNARE partners in the fusion reaction, I tested the sensitivity of homotypic fusion to inhibition of SNARE function through anti-SNARE antibodies. If new SNAREs compensate for the loss of the silenced one, antibodies against them would block fusion in the knock-down, but not in the control endosomes. Since the Qa-SNARE syntaxin 13 is the best-characterized endosomal SNARE, I performed these experiments using material from control and syntaxin 13 knock-down cells. I compared the effects of antibodies targeting a variety of Q- and R-SNAREs (Figure 3.16D). Only a few differences could be seen between syntaxin 13 knock-down and wild type organelles (such as a more efficient block via VAMP4 antibodies, and a less efficient one by syntaxin 16 antibodies), with no new Qa-SNARE appearing to take over syntaxin 13's function. Taken together, these observations further weaken the hypothesis of new SNARE molecules substituting for the downregulated proteins and favor a model of abundance in SNARE availability. 

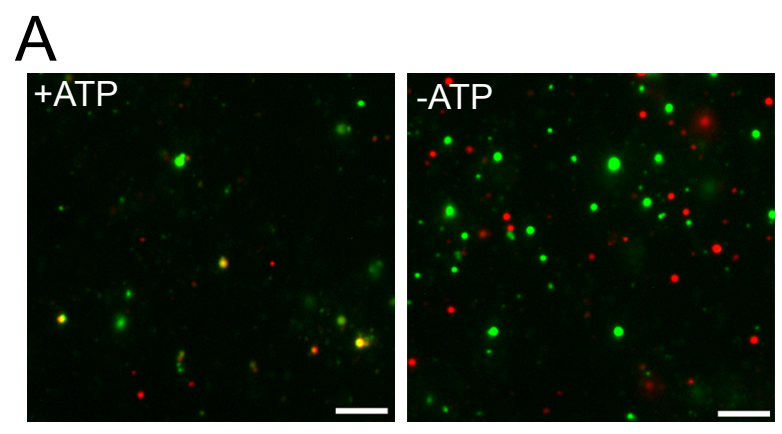

B
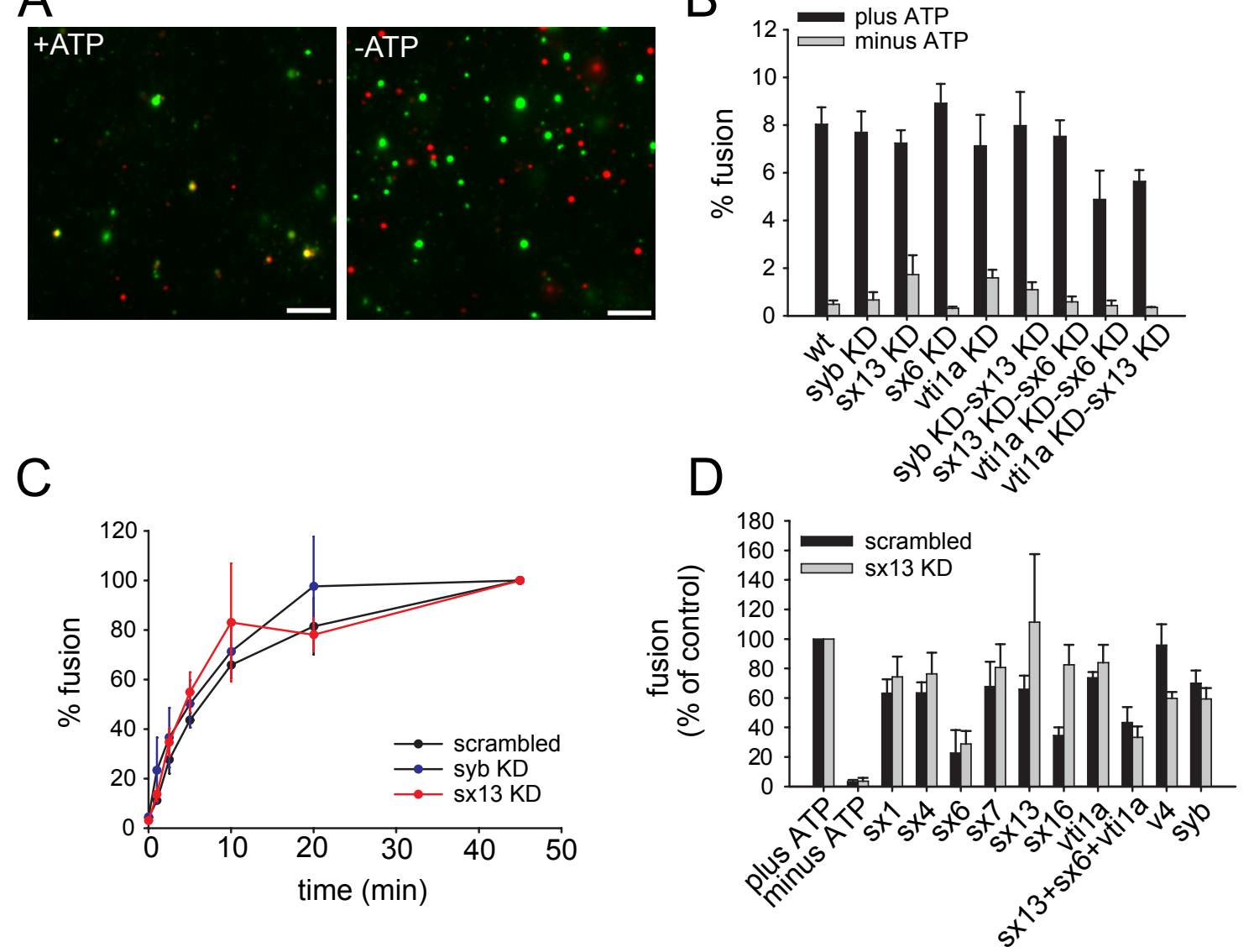

Figure 3.16: Downregulation of individual SNAREs does not affect the fusion of early endosomes in vitro. A) Wild type PC12 cells and knock-down cell lines were loaded for 5 min with Alexa 488-labeled (green) or Alexa 594-labeled (red) dextran. Cells were then homogenized and post-nuclear supernatant (PNS) containing the labeled organelles was isolated. The two differentially labeled PNSs were incubated in presence of cytosol and in presence or absence of ATPregenerating system, for $45 \mathrm{~min}$ at $37^{\circ} \mathrm{C}$. Reactions were spun down on coverslips and visualized. Representative images from the fusion of green-labeled endosomes from syntaxin 13 knock-down cells and red-labeled endosomes from syntaxin 6 knock-down cells are shown (green channel, Alexa 488; red channel; Alexa 594). Scale bar: $5 \mu \mathrm{m}$. B) Fusion is expressed as the percentage of green organelles colocalizing with red ones. Quantification of three independent experiments (means \pm s.e.m) is presented. C) Fusion reactions using PNS from scrambled, syntaxin 13 and synaptobrevin KD cell lines were performed for various time intervals. Maximum percentage of fusion after 45 min for each cell line was normalized to $100 \%$. The value at each time point is the mean \pm s.e.m. from three individual experiments. D) Fusion reactions using PNS from either control transfected or syntaxin $13 \mathrm{KD}$ cell lines were performed in presence of polyclonal antibodies against various SNAREs (means \pm s.e.m. from three individual experiments). 


\subsubsection{SNARE associations persist in the knock-down background, de- spite the low SNARE amounts}

For fusion to occur, the amount of SNARE complexes, rather than the actual availability of single SNARE molecules, is the limiting factor. Since endosomal fusion was not affected in knocked-down cells, it can be hypothesized that the residual SNARE amounts are still able to form endogenous SNARE complexes within the endosomal membrane. It has been already discussed that endosomal SNAREs form cis-complexes in a promiscuous manner, whereby their relative abundances correlate with the abundance of the participating SNAREs in the membrane in a mass-action manner. Thus, one can expect that a reduction of a given SNARE results in a concomitant reduction of the corresponding SNARE complexes, which may allow other subfamily members to substitute in complex formation. To test this assumption, I used the established system of SNARE interactions on early endosomes to study SNARE associations under the new stoichiometry established by the siRNA downregulation.

I immunoprecipitated SNARE complexes with an antibody against syntaxin 13, using solubilized PNS from control and synaptobrevin knock-down cells as starting material. I chose the non-cognate SNARE synaptobrevin for the study because I showed earlier that this SNARE is more than 100-fold more abundant than the endosomal SNAREs and dominates cis-SNARE complexes formed by endosomal Q-SNAREs. As expected, the reduction in the expression of the R-SNARE synaptobrevin allowed syntaxin 13 to interact more with its own cognate R-SNARE VAMP4 (Figure 3.17A). The observation was further confirmed when I immunoprecipitated VAMP4 and found 2.5 times more syntaxin 13 in the precipitates of the synaptobrevin knock-down PNS (data not shown). Similarly, I immunoprecipitated synaptobrevin from control and syntaxin 13 knock-down PNS fractions. Even though the amount of synaptobrevin precipitated from the two preparations was the same, slightly more syntaxin 1 (which is a Qa SNARE, like syntaxin 13) was pulled down from the syntaxin $13 \mathrm{KD}$ cells compared to the control (Figure 3.17B).

On one hand, these findings confirm our initial hypothesis that mass-action indeed governs cis-complex formation and they suggest that, upon downregulation of an 

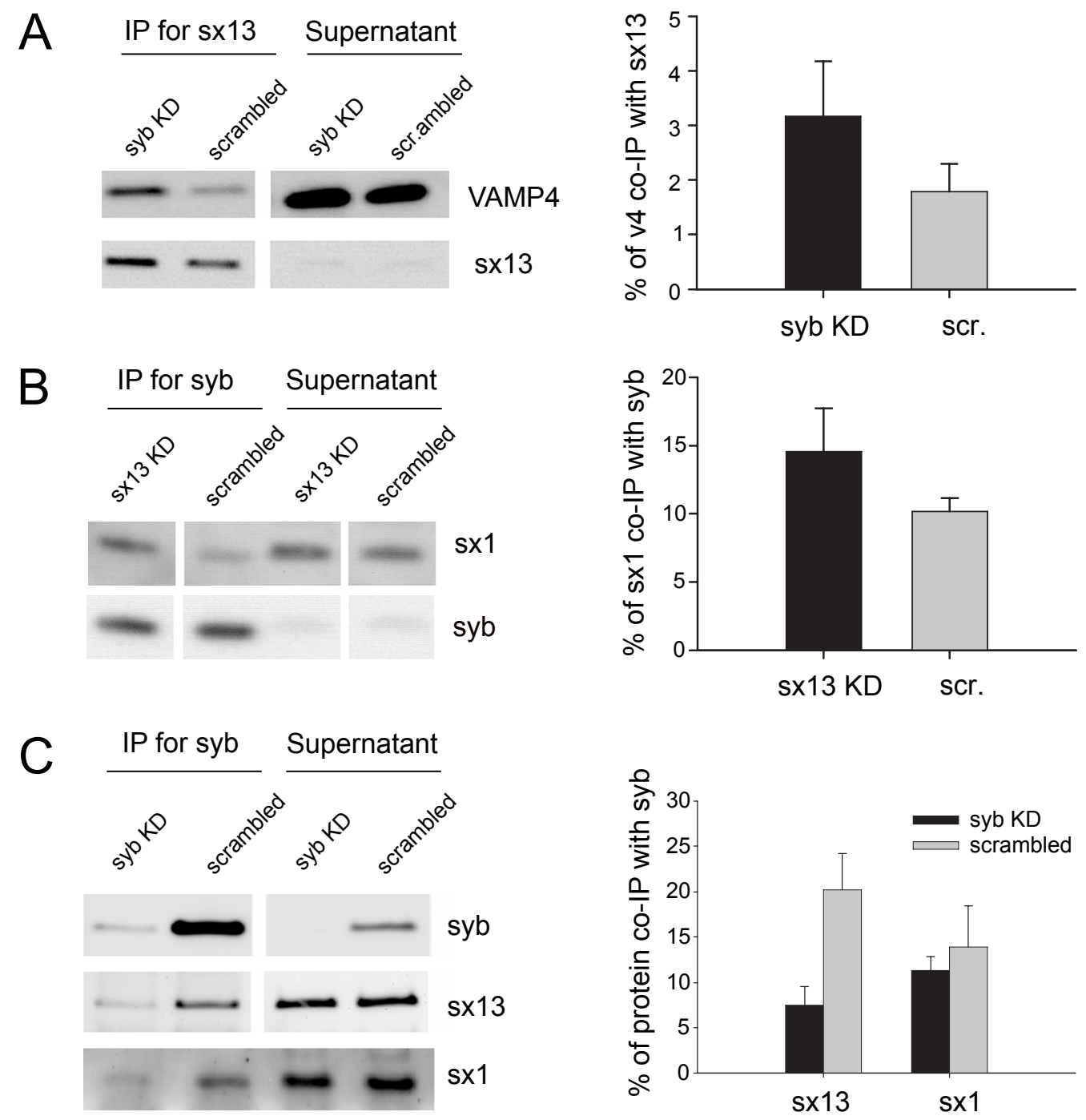

Figure 3.17: Changes in cognate SNARE associations upon downregulation of noncognate partners. A) PNS from control and syb KD cells was incubated under ATP-depleting conditions for 45 min at $37^{\circ} \mathrm{C}$, was solubilized and immunoprecipitation was performed with an antibody against syntaxin 13 . VAMP4 and syntaxin 13 were detected in the precipitates by immunoblotting. $10 \%$ of the precipitates and $1 \%$ of the supernatants were loaded. The percentage of co-precipitated VAMP4 from control and KD extracts is presented after normalization to the starting material and to the amount of precipitated syx 13. B) Immunoprecipitation with an antibody against synaptobrevin was performed using solubilized PNS from control and syx 13 KD cells. The amount of co-immunoprecipitated syntaxin 1 was calculated as before. C) To specifically quantify the strength of interactions upon knock-down, purified organelles were used in this experiment. PNS from control and syb KD cells was incubated under ATP-depleting conditions and loaded on discontinuous sucrose gradient. A band enriched in early endosomes was isolated and solubilized. Synaptobrevin was immunoprecipitated and the presence of syntaxins 1 and 13 was identified in the precipitates. A total of $20 \%$ of the precipitates and $2.5 \%$ of the supernatants were loaded. The amounts of co-immunoprecipitated syntaxins were calculated as above. Averages \pm s.e.m. from three independent experiments are presented for all cases (A-C). 
abundant non-cognate SNARE, interactions between cognate partners are enhanced. On the other hand, it is rather striking that the impact of SNARE downregulation on SNARE interactions does not correlate with the efficiency of the knock-down. In wild type cells, syntaxin 13 shows a strong preference for the non-cognate SNARE synaptobrevin, instead of its cognate partner VAMP4, because of the high synaptobrevin abundance. Interestingly, when synaptobrevin levels are now decreased 10-fold, the interaction between syntaxin 13 and VAMP4 is enhanced only by a factor of two.

This issue became more apparent when I measured the amount of syntaxin 13 and syntaxin 1 that co-immunoprecipitated with synaptobrevin from early endosomes of control and synaptobrevin knock-down cells (Figure 3.17C). Approximately 20-times less synaptobrevin was pulled down from the knock-down cells compared to control. In the same preparation, I found only 2.5-times less syntaxin 13 and almost identical amounts of syntaxin 1 co-immunoprecipitating with synaptobrevin compared to the control. These levels are strikingly high considering the low amount of synaptobrevin pulled down from the knock-down extracts. These numbers suggest that the low levels of SNAREs persisting after knock-down maintain (cis) SNARE-SNARE interactions at a much higher level than expected.

Thus, it is apparent that low amounts of synaptobrevin can engage substantial amounts of syntaxin molecules. Is this the case also in wild type cells, i.e. does a small sub-population of synaptobrevin molecules account for all of the interactions with the syntaxins? Based on the known stoichiometries of SNAREs in the endosome membrane, one can calculate the amount of synaptobrevin necessary to pull down the observed levels of, for example, syntaxin 13 ( 19\%, Figure 3.17). To investigate this, I used the Monte Carlo simulation that was described in section 3.1.2 where I inserted the early endosomal and exocytic SNAREs in their stoichiometric proportions on a model endosome, and allowed all proteins to associate in cognate and non-cognate complexes. According to the simulation, $100 \%$ of syntaxin 13 could be associated with synaptobrevin, due to the great abundance of this R-SNARE (Figure 3.18, first column). To reach the experimentally observed levels of syntaxin 13 pull-down, the synaptobrevin levels need to be reduced to as low as 


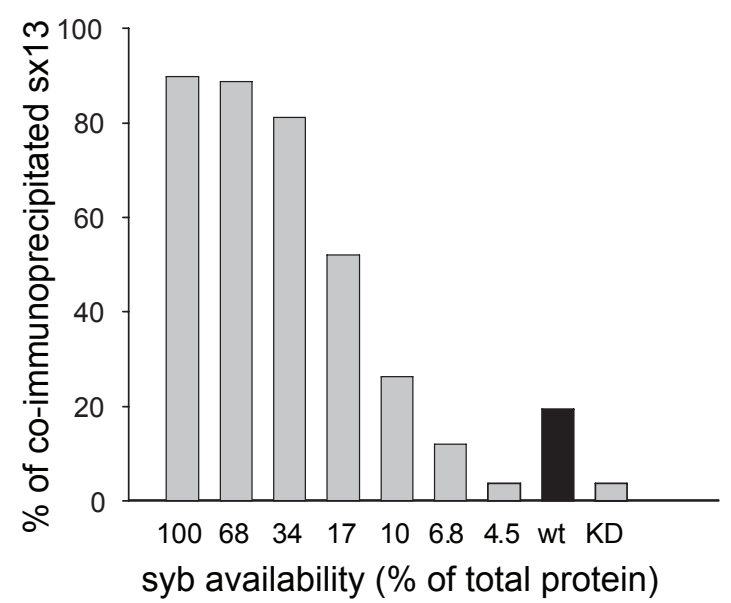

Figure 3.18: Less than $\mathbf{1 0 \%}$ of synaptobrevin binds to syntaxin 13. I simulated the interaction of synaptobrevin with syntaxin 13 on a model endosome of $\sim 233 \mathrm{~nm}$ in diameter, containing syntaxin 13, VAMP4, synaptobrevin and the exocytic acceptor complex (syntaxin 1/SNAP 25) in numbers corresponding to the actual amounts of the proteins on the early endosomes (see section 3.1.2). The amount of synaptobrevin available for complex formation varied from $100 \%$ to $4.5 \%$ of total synaptobrevin amount. I allowed the SNAREs to mix and interact for 1000 iterations for each of the different synaptobrevin amounts, after setting the affinity of R-SNAREs for their noncognate partners to $1 / 20$. The affinity for the cognate SNAREs was 1 . I counted the number of syntaxin 13/synaptobrevin complexes under the different conditions of synaptobrevin availability (grey bars). The percentage of syntaxin 13 in complex with synaptobrevin on early endosomes from wild type and synaptobrevin knock-down cells is also shown (black and grey bars) from averages of the experiments shown in Figure 3.17, normalized to the amount of precipitated synaptobrevin.

$8 \%$, demonstrating that only a minor fraction of the R-SNARE is necessary for this interaction. Assuming that only a small portion of the available SNARE molecules generally participate in SNARE interactions, it is certainly not surprising that even highly efficient SNARE knock-downs result in very minor phenotypes.

\subsubsection{SNARE clustering on the endosomal membrane}

From the data presented so far, it is apparent that SNAREs are not participating in the maximum of potential interactions as expected from their membrane concentration. To further investigate this observation, I studied the distribution of SNAREs on the endosomal membrane. I transfected wild type PC12 cells with the GFP-Rab5-Q79L, to induce the formation of enlarged endosomes, and I per- 
formed immunostainings for the different SNAREs. As indicated in Figure 3.19, all of the SNAREs studied (both the endosomal SNAREs and synaptobrevin) exhibit a 'spotty' distribution, with the majority of the proteins concentrated in distinct clusters. Focusing on synaptobrevin, I also stained for the protein in synaptobrevin knock-down cells transfected with GFP-Rab5-Q79L, and observed a more diffuse protein distribution. The observation was further confirmed when I performed auto-correlation analysis of the intensity profiles of synaptobrevin staining along GFP-Rab5-positive endosomes from wild type and knock-down cells. The position of the first maximum of the auto-correlation trace was used as a read-out of the inter-cluster distances. The synaptobrevin staining on wild type endosomes exhibited larger values, indicative of the existence of distinct protein clusters. The observed change in synaptobrevin distribution is not due to a poor detection of the residual synaptobrevin in knock-down cells, since the signal at the synaptobrevin channel in knock-down cells was still significantly stronger than the negative controls (Figure 3.19B). From the experimental results and the modeling calculations, it can be suggested that the clusters in wild type cells represent inactive (or reserve) pools of SNAREs, which are restricted from interacting with other partners - thus allowing the small and more diffuse amounts of SNAREs from the knock-downs to form almost as many interactions as the (small) free pools of SNAREs in the wild type cells.

This hypothesis also predicts that a dispersal of SNAREs from the clusters would be accompanied by an increase in SNARE-SNARE interactions. The means by which SNAREs form clusters are relatively complex (Sieber et al., 2006, 2007) and therefore interfering with cluster organization is not trivial. However, it has been shown for several SNARE molecules (including one of our SNAREs of interest, synaptobrevin) that they reside in cholesterol-containing microdomains, and that depletion of cholesterol disrupts this organization and interferes with their function (Chamberlain et al., 2001; Lang et al., 2001; Predescu et al., 2005). Work from Dr. Silvio Rizzoli (European Neuroscience Institute) showed that depletion of cholesterol from purified early endosomes leads to a dispersal of synaptobrevin clustering (Figure 3.20A). Therefore, I further tested the effects of cholesterol de- 
A
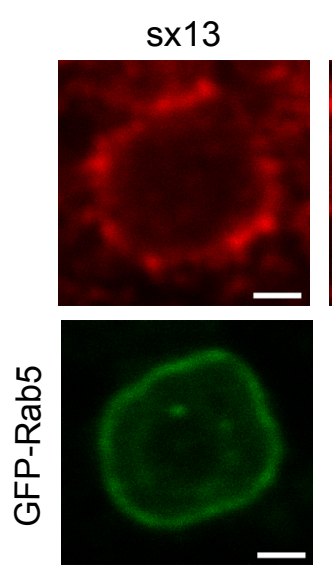

B
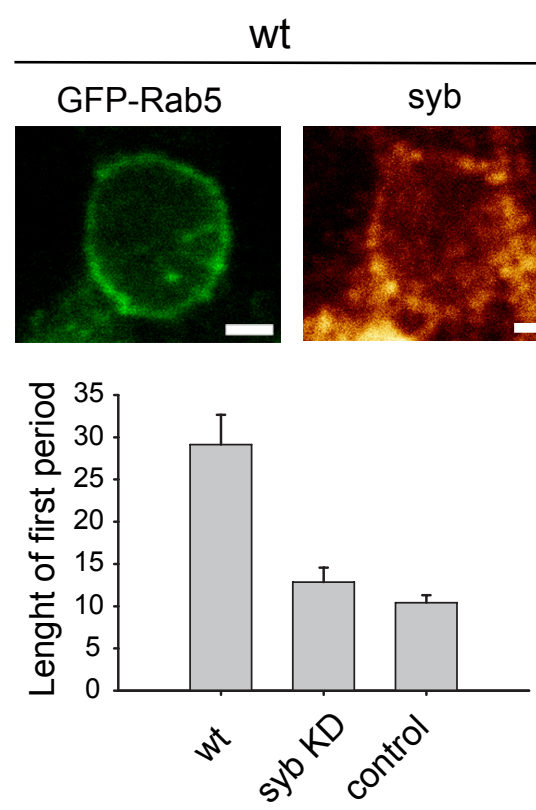

sx6

wt

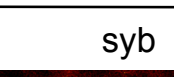

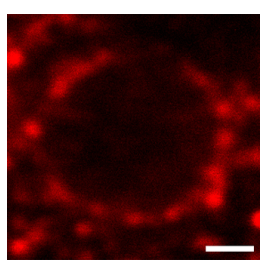
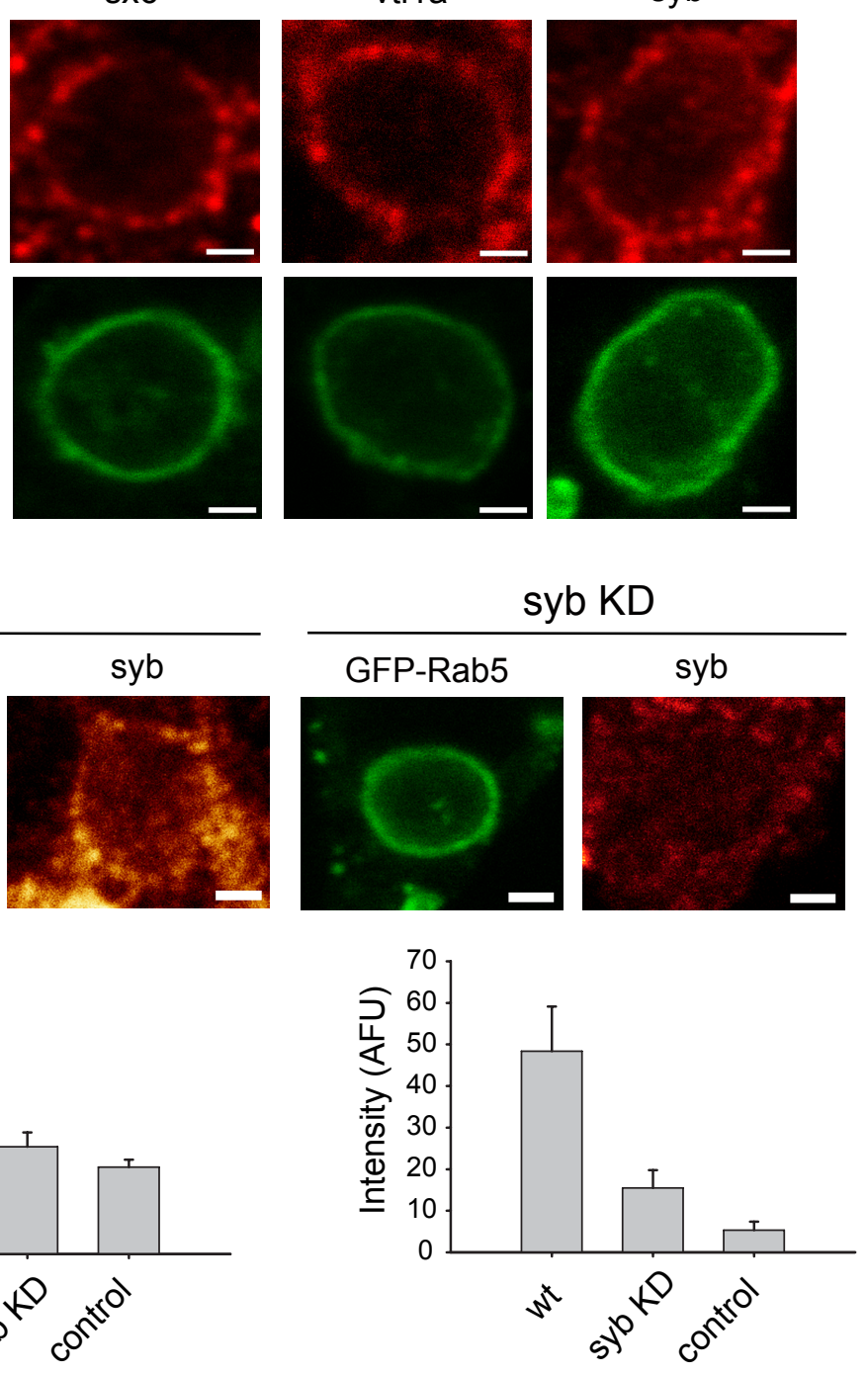

Figure 3.19: SNAREs are clustered on the endosomal membrane. A) Wild type PC12 cells were transfected with GFP-Rab5 Q79L. Cells were fixed, immunostained for syntaxin 13, 6, vti1a, and synaptobrevin and imaged with confocal microscopy. Typical images of enlarged endosomes are presented. Green channel: GFP-Rab5 Q79L; red channel: stained protein. Scale bar: $1 \mu \mathrm{m}$. B) Typical images of enlarged endosomes from wild type and synaptobrevin KD cells are presented. Scale bar: $1 \mu \mathrm{m}$. The intensity profile of the synaptobrevin staining along the endosomal membrane was obtained for $10-15$ endosomes per cell type per experiment. The periodicity of the signal was quantified by taking the position of the first maximum of the autocorrelation function for each endosome in pixels (left graph). The average intensity of the synaptobrevin signal along the membrane was also calculated (right graph). For the control sample, only the fluorescently-labeled, secondary antibody was used for staining. Averages \pm s.e.m. from three independent experiments are shown. 
pletion on synaptobrevin's SNARE interactions. I incubated PNS from PC12 cells in presence or absence of $\beta$-methyl-cyclodextrin (a drug that depletes cholesterol from membranes) and performed immunoprecipitation using an antibody against synaptobrevin. Confirming the initial hypothesis, cholesterol depletion increased the amount of syntaxin 13 co-immunoprecipitating with synaptobrevin by about three-fold (Figure 3.20), thus demonstrating that the exocytic R-SNARE is indeed restricted (probably in membrane clusters) from interactions in the wild-type situation, despite its great abundance.

\subsubsection{The role of fusion-docking co-regulation}

The SNAREs are not the only molecules functioning in fusion, with the up-stream processes of tethering and docking being also essential. As already discussed in $\mathrm{s}$ 3.1.3, important proof-reading mechanisms ensuring the cognate SNARE pairing are taking place during the docking stage. In the endosomal system, the GTPase Rab5 and its effectors are the main tethering modulators, with Rab5 organizing molecular microdomains where tethering and fusion factors are recruited for a co-operative function (Zerial and McBride, 2001). Importantly, the endosomal SNARE syntaxin 13 has been shown to participate in these macromolecular clusters via its direct interactions with the Rab5 effector EEA1 (McBride et al., 1999).

What is the significance of this interaction and how is the docking machinery affected by the downregulation of its downstream fusion components? Immunostaining of control and syntaxin 13 knock-down cells showed that the total levels of EEA1 were not changed upon syntaxin 13 downregulation (the EEA1 intensity of syntaxin 13 knock-down cells was about $86 \%$ of the wild type; Figure 3.21A). Since EEA1 can be found both in the cytosol or on the endosomal membrane, depending on its recruitment by the docking machinery, I checked its distribution in the wild type and syntaxin 13 knock-down background. PNS fractions from the two cell lines were incubated under ATP-regenerating conditions at $37^{\circ} \mathrm{C}$ for $45 \mathrm{~min}$. Reactions were then centrifuged to separate soluble material from membranes and the content of each fraction in EEA1 was checked by immunoblotting. As shown in Figure 3.22, syntaxin 13 knock-down endosomes contained more membrane-bound EEA1, while 

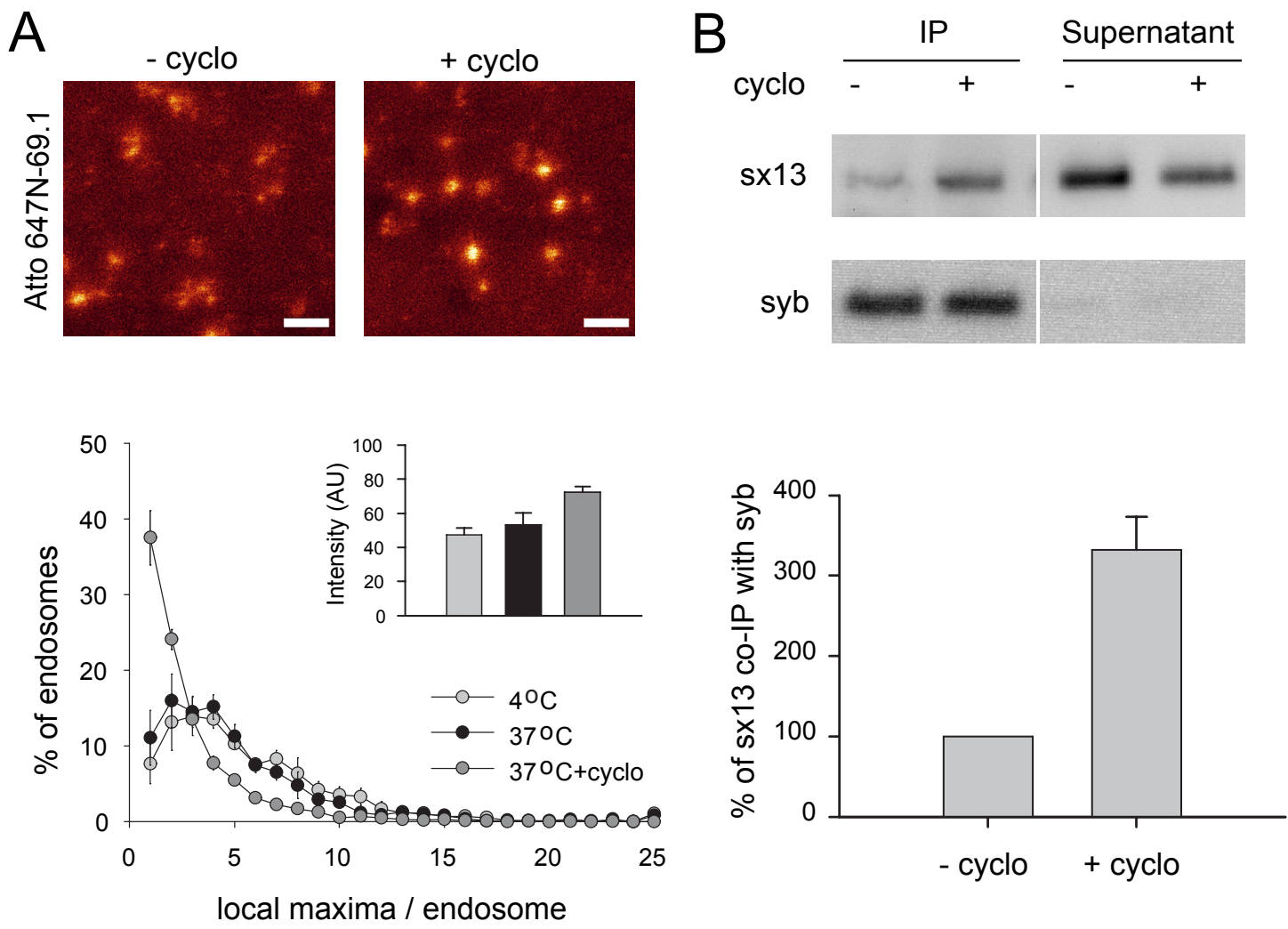

Figure 3.20: SNAREs are restricted from interactions due to clustering on the endosomal membrane. A) Purified endosomes from PC12 cells were incubated for 30 min either at $4^{\circ} \mathrm{C}$ or at $37^{\circ} \mathrm{C}$ in presence or absence of $60 \mathrm{mg} / \mathrm{ml} \beta$-methyl-cyclodextrin. They were then fixed and stained for synaptobrevin using the monoclonal antibody 69.1 and an Atto 647N-labeled secondary antibody and imaged using STED microscopy. Typical images of stained endosomes incubated at $37^{\circ} \mathrm{C}$ with $(+$ cyclo) or without $(-$ cyclo $) \beta$-methyl-cyclodextrin are shown. Scale bar: $0.5 \mu \mathrm{m}$. The synaptobrevin clusters per endosomes were quantified for each condition and plotted as a histogram. The intensity of the staining was also calculated (inset graph). Averages \pm s.e.m. from three independent experiments are presented. B) PNS from wild type or control cells was incubated under ATP-regenerating conditions for $30 \mathrm{~min}$ at $37^{\circ} \mathrm{C}$, in absence or presence of $60 \mathrm{mg} / \mathrm{ml} \beta$-methyl-cyclodextrin. After addition of $2 \mathrm{mM} \mathrm{NEM}$, the PNS was further incubated for 20 min, solubilized and immunoprecipitation was performed with an antibody against synaptobrevin. Syntaxin 13 was detected in the precipitates by immunoblot analysis. A total of $20 \%$ of the precipitates and $2 \%$ of the supernatants are loaded and the percentage of the co-precipitated syntaxin 13 was calculated after normalizing to the amount of precipitated synaptobrevin (averages \pm s.e.m. from three independent experiments). 


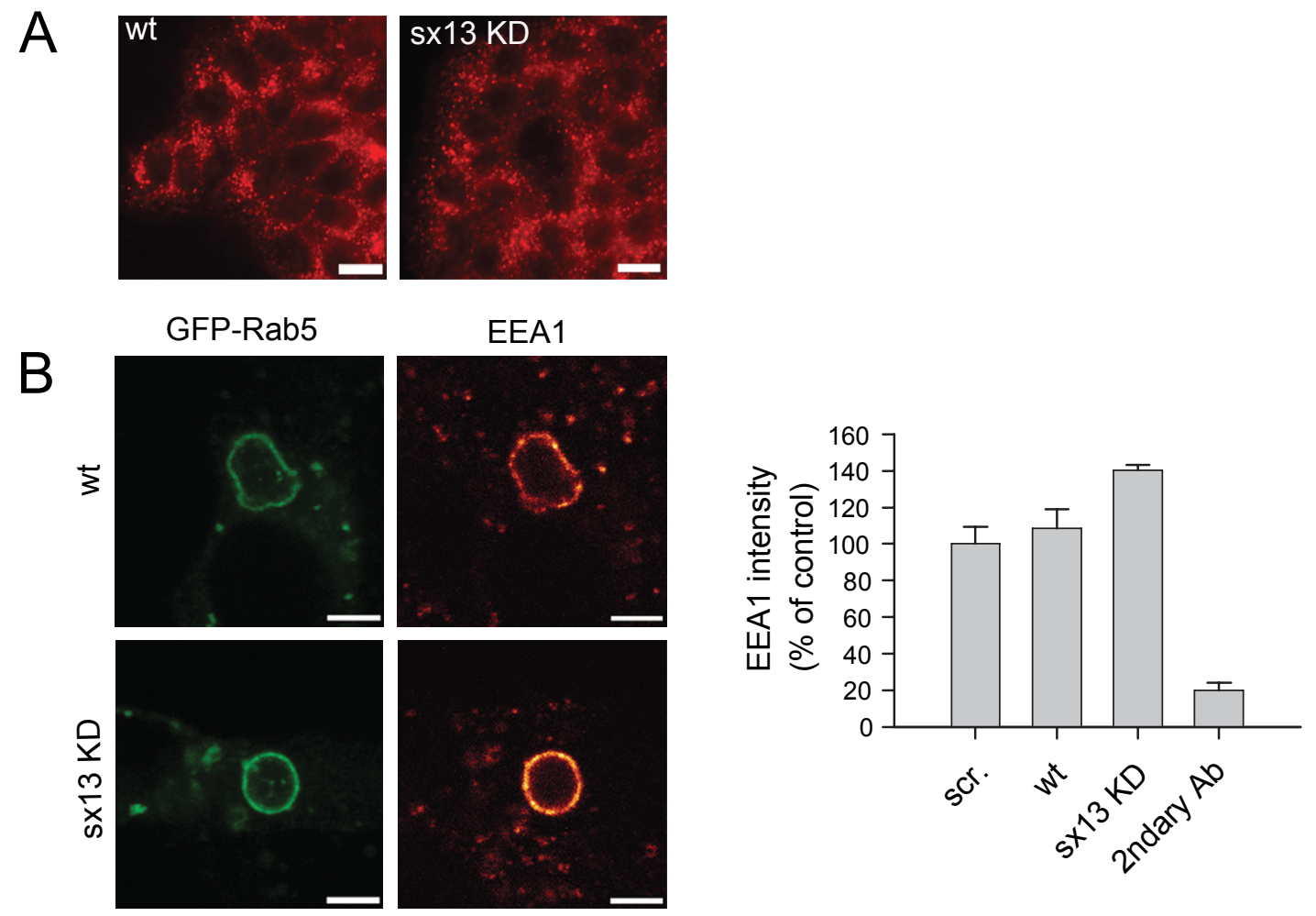

Figure 3.21: Enhanced recruitment of EEA1 on syntaxin 13 KD endosomes. A) Wild type and syntaxin 13 knock-down cells were immunostained against EEA1 (red). Scale bar: $10 \mu \mathrm{m}$. B) Wild type, control transfected and syntaxin 13 knock-down cells, expressing GFP-Rab5 Q79L were immunostained with a monoclonal antibody against EEA1 and imaged with confocal microscopy. Typical images of enlarged endosomes from wild type and syntaxin 13 knock-down cells are presented. Scale bar: $3 \mu \mathrm{m}$. The intensity of the EEA1 on the membrane of the enlarged endosomes was quantified and normalized to the intensity of GFP-Rab5. 10 images were acquired per cell line per experiment. Averages \pm s.e.m. from three independent experiments are shown, the EEA1 intensity from control transfected cells was set to $100 \%$.

the distribution of Rab5 remained unaltered. I also observed a similar trend for the EEA1 binding to membranes for the other knock-down lines (Figure 3.22).

These observations were further supported when Rab5-Q79L-positive organelles were stained for EEA1 in control and syntaxin 13 knock-down cells and imaged using confocal microscopy. The EEA1 intensity pattern along the endosomal membrane revealed higher intensity for the syntaxin $13 \mathrm{KD}$ organelles, consistent with the increased membrane recruitment of the protein (Figure 3.21). 

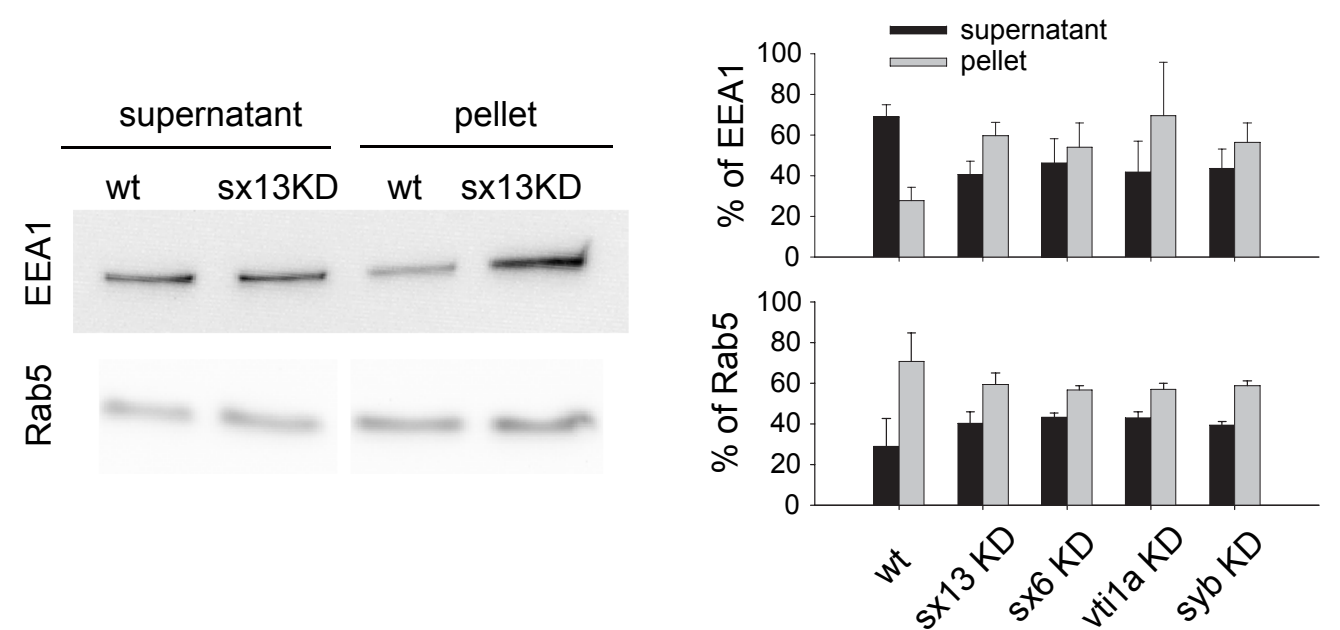

Figure 3.22: More EEA1 is recruited on the membrane of knock-down organelles. PNS from wild type and sx13, sx6, vti1a and syb knock-down cells was incubated under ATP regenerating conditions for $45 \mathrm{~min}$ at $37^{\circ} \mathrm{C}$. Reactions were centrifuged, supernatants were removed and pellets were solubilized. $10 \%$ of supernatants and pellets were loaded and immunoblotted for EEA1 and Rab5. A typical example of an EEA1 and Rab5 blot for wt and sx13 PNS is shown. The percentage of EEA1 and Rab5 in supernatant and pellet was quantified for each cell line. Averages \pm s.e.m. from three independent experiments are shown.

Since EEA1 is a central player of the docking machinery, I asked whether the increase in membrane-bound EEA1 on syntaxin 13-deficient organelles also results in enhanced docking of these endosomes. To estimate docking, I performed in vitro fusion reactions, as already described above (section 2.2.7), using control and syntaxin 13 knock-down fluorescently labeled PNS. I subsequently calculated the distance between each green organelle and a neighboring red one and plotted the distribution of the distances between the organelles. Typically, the curves show two peaks, corresponding to fused and docked organelles respectively. Organelles that lay at a distance (from each other) lower than $112 \mathrm{~nm}$ are considered fused, whereas endosomes that are $137-512 \mathrm{~nm}$ apart are docked (Geumann et al., 2008). The differences observed among the fusion and docking peaks for the different cell lines are not informative since the absolute amount of fusion between different PNS preparations cannot be directly compared, due to experimental variations. To get a more 

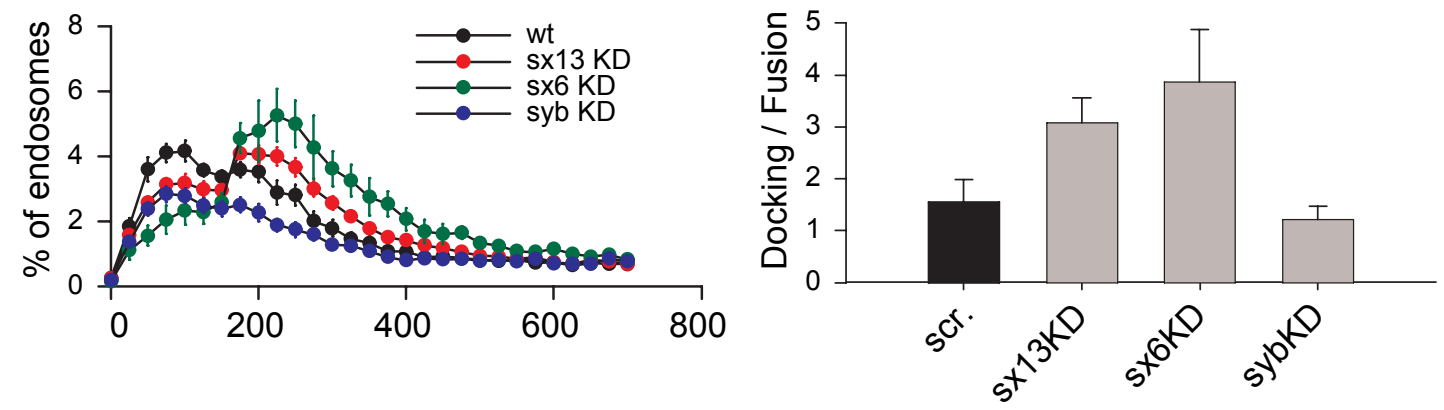

Figure 3.23: Docking is enhanced upon silencing of early endosomal SNAREs. Fusion reactions using PNS from control transfected and knock-down cells were performed. The distance between each green organelle and a neighboring red one was calculated and the distribution of the distances between the organelles was plotted (left graph). The first peak corresponds to fused organelles, the second to docked ones. The ratio of docked to fused organelles was also calculated and presented (right graph). Averages \pm s.e.m. from three to twelve independent experiments are shown.

quantitative view, I measured the ratio between the amounts of docked and fused endosomes in the different cell lines (Figure 3.23, right graph). Knock-down of the early endosomal SNAREs (syntaxin 13, syntaxin 6) increased dramatically the proportion of the docked organelles, while, as expected, synaptobrevin (the noncognate, exocytic SNARE) knock-down left them unaffected. In summary, these results suggest a second mechanism alleviating the SNARE knock-down phenotype. The enhanced function of the docking machinery might serve as a compensatory mechanism of possible fusion impairment caused by the SNARE downregulation and therefore explain the lack of negative phenotypes in endosomal function at the knocked-down cells. It is an additional example of two cellular mechanisms (docking and fusion) functioning co-operatively to ensure intact cellular function. 


\section{4}

\section{DisCUSSION}

Excluding the plethora of molecules that mediate the initial recognition and tethering of two compartments, the SNAREs are the key mediators of membrane fusion. Members of a highly conserved protein family, SNAREs are present in all intracellular compartments and promote the various fusion processes, by following a common mechanism: the SNARE molecules that are present in two opposing membranes come in association, bring the membranes in proximity and facilitate lipid mixing. Different SNARE molecules are believed to function in fusion events that involve different organelles. Nevertheless, the continuous membrane mixing taking place during intracellular transport results in a redistribution of SNAREs in compartments where they do not exhibit their function.

The promiscuity that SNARE molecules exhibit in complex formation both in vitro and in vivo raises the question on how only the cognate SNAREs interact with each other for fusion and how non-cognate molecules are functionally 'silenced'. Early endosomes are a suitable system to investigate this problem, since they are found in the center of the endocytic pathway, constantly exchange material with a variety of organelles and thus, accumulate a variety of different SNARE molecules. Nevertheless, it has been shown that only the early endosomal SNAREs mediate the homotypic fusion of these organelles, despite the plethora of other SNARE isoforms present on their membrane.

In the present study, I investigated the early endosomal fusion and the SNAREs involved in this process and proposed new mechanisms for the regulation of the specificity in SNARE pairing and function. I showed that cognate and non-cognate SNAREs occupy the same microdomains on the endosomal membrane and that the specificity is ensured only by the co-operative function of two mechanisms: the 
preference for cognate interactions when SNARE molecules associate in a transconfiguration and the lateral segregation of SNAREs, with cognate isoforms enriching at fusion sites and non-cognate ones being excluded. The high level of regulation revealed by the fusion machinery led me to further challenge its robustness and study its adaptation abilities under stress conditions, such as low availability of SNARE molecules. To this end, I downregulated the expression of early endosomal and exocytic SNAREs by siRNA means and I observed no effect in cell viability and intracellular trafficking. SNARE interactions were not altered in knocked-down cells and the docking machinery exhibited enhanced activity. These results provide an additional insight in the regulation of endosomal fusion and trafficking, suggesting that SNARE abundance and the tight co-operation among docking and fusion factors serve as a safety net for intracellular fusion under stress conditions.

The investigation of the mechanisms responsible for the specificity and robustness of the fusion machinery revealed that the efficient regulation of a complex intracellular process such as fusion can only be achieved via the combinatorial action of multiple molecules and processes. The function of factors involved in docking and priming, upstream of fusion, together with the abundance of SNARE molecules available, are combined in an highly orchestrated and combinatorial manner, to mediate intracellular trafficking.

\subsection{Regulation of specificity in SNARE-mediated fusion}

The great variety of SNARE molecules residing on the endosomal membrane and the powerful contribution of mass action to the identity of cis-SNARE complexes require the existence of highly efficient and precise mechanisms that regulate SNARE pairing specificity for fusion.

The Monte-Carlo simulations showed that due to the overwhelming abundance of synaptobrevin, non-cognate complexes among exocytic and endosomal SNAREs are expected to dominate. Even if a 100-fold preference for cognate interactions is hypothesized (in contrast to experimental data that suggest no affinity differences between cognate and non-cognate SNAREs (Fasshauer et al., 1999)), cis-interactions are condemned to non-cognate complex formation, virtually irrespective of SNARE 
preference. Nevertheless, the results presented in this thesis suggest the function of a selectivity filter that can overcome the power of mass action and can promote the interaction of only cognate SNAREs in trans. However, the exact molecular machinery mediating SNARE pairing specificity has not been unraveled. Multiple lines of evidence though suggest that proteins of the SM family or tethering factors may play a role.

\subsubsection{SM proteins contribute to SNARE pairing specificity}

\subsubsection{SM proteins control SNARE complex assembly}

The preference for cognate SNARE associations in trans was demonstrated when soluble recombinant SNAREs were added externally and interacted with membraneresident SNAREs from PC12 cells (Bethani et al., 2007). The early endosomal syntaxin 13 or the late endosomal syntaxin 7 were not able to compete for the binding of the cognate syntaxin 1 to the endogenous, membrane-associated SNAP-25. Similarly, the exocytic synaptobrevin exhibited clearly higher-affinity associations with membrane SNAREs compared to the non-cognate, late endosomal R-SNARE endobrevin. These experiments were performed under conditions that did not allow the formation of preparatory docking complexes, which may naturally occur prior to fusion. Thus, an intrinsic selectivity filter must be responsible for the observed ability of the exocytic, membrane SNAREs to discriminate between cognate and non-cognate partners in trans.

Studying SNARE pairing selectivity in the homotypic fusion of early endosomes, I demonstrated the existence of a second regulatory mechanism, that functions via the spatial accumulation of cognate SNAREs and the simultaneous exclusion of non-cognate partners from fusion sites. Nevertheless, simulation of the interactions among early endosomal SNAREs at the interface of two endosomes revealed that only the combinatorial action of spatial segregation and a selectivity filter for transSNARE interactions can ensure the formation of productive SNARE complexes and endosomal fusion. But what is the nature of such a selectivity mechanism?

Candidates for this regulatory role are the members of the conserved Sec1/Munc18 family, the SM proteins. Although their specific mode of action is 
a topic of high controversy, SM proteins are undoubtedly integral partners of the fusion machinery and regulators of SNARE function. They bind syntaxins and SNARE complexes and upon inactivation of any member of the family, complete block of the respective transport step (and occasionally cell death) is observed (Bryant and James, 2001; Novick and Schekman, 1979; Verhage et al., 2000). Interestingly, in contrast to the broader distribution of SNAREs, SM proteins are highly enriched at fusion sites (Carr et al., 1999; Zilly et al., 2006) and were shown to participate in vesicle docking, indicating their potential contribution to the specificity of membrane fusion.

Even though SM proteins that function specifically at the early endosomal compartment have not been characterized, the possibility that the early endosomal SNARE complex or monomeric syntaxin 13 (which possesses the basic characteristics of a syntaxin) associate with a member of the SM family cannot be excluded. SM proteins have been shown to function in several fusion processes (see for example Bryant and James (2003); Dulubova et al. (2007); Khvotchev et al. (2007); Kosodo et al. (2002)). Direct associations between SM proteins and SNARE complexes have been reported for the ER-Golgi and TGN-endosome trafficking (Coe et al., 1999; Latham et al., 2006; Peng and Gallwitz, 2002), as well as for exocytosis (D'Andrea-Merrins et al., 2007; Togneri et al., 2006) in yeast and mammals. In particular, SM proteins were shown to promote and regulate the specificity of SNARE complex assembly. For example, the yeast SM protein Sly1p promotes the association of Sed5p in the ER-to-Golgi core SNARE fusion complex, contributing to the formation of functional cognate complexes (Peng and Gallwitz, 2002). Similarly, Sec1p, which participates in exocytosis in yeast, stimulates membrane fusion by binding to pre-assembled SNARE complexes (Scott et al., 2004).

Therefore, a possible mode of action for the observed selectivity filter could be its ability to recognize and stabilize the cognate, trans-complexes, ensuring their availability for fusion. This idea is supported by experiments where liposomes containing syntaxin 1/SNAP-25 preferentially fuse with liposomes containing the cognate SNAREs VAMP2 or VAMP8 in presence of Munc18, thereby losing the promiscuous fusogenic ability that they exhibit in absence of the SM protein (Shen et al., 2007). 
In addition, experiments using plasma membrane lawns from PC12 cells showed that synaptobrevin binding to syntaxin1/SNAP-25 can displace Munc-18, whereas endobrevin is much less efficient (Zilly et al., 2006).

\section{The role of SM proteins in docking, sorting and SNARE stabilization}

Considering that the proposed specificity mechanism of local SNARE re-distribution occurs during docking, it is possible that selectivity of SNARE pairing also functions during this pre-fusion step. Interestingly, SM proteins have been involved in tethering and docking, as well as in SNARE stabilization and sorting.

For instance, the levels of Tlg2p and syntaxin 1 are decreased when Vps45p and Munc18-1 are eliminated (Bryant and James, 2001; Verhage et al., 2000), while the yeast ER syntaxin Ufe1 is protected from degradation when bound to the SM protein Sly1 (Braun and Jentsch, 2007). Additionally, syntaxin association with SM proteins promotes the transport of the newly-synthesized syntaxins to their organelle of residence, possibly by preventing their engagement in non-functional SNARE complexes during transport and by regulating their proper sorting (Arunachalam et al., 2008; Medine et al., 2007). Elimination of SM proteins not only blocks fusion but also results in the accumulation of undocked vesicles, as observed upon knock-out of Munc-18 (Voets et al., 2001), indicating a role of SM proteins in vesicle docking. This possibility is further supported by the fact that multiple interactions have been reported between SM proteins and tethering factors (Nielsen et al., 2000; Seals et al., 2000). Interestingly, for SM proteins involved in endosomal/vacuolar fusion, this is the only (indirect) mode of interaction with the SNARE machinery known till today. Vps33p and Vps45p are part of the multimeric HOPS tethering complex, which is recruited to the membrane by the Rab protein Ypt7p (Price et al., 2000; Ungermann et al., 2000), while there are even indications of direct binding between Rab3a and Munc18 (van Weering et al., 2007).

Even though the diversity of functions that SM proteins exhibit qualify them as possible regulators of SNARE pairing specificity, their direct involvement in early endosomal fusion has not been demonstrated. The exact mechanisms that allow SNAREs to select their cognate partners when presented in trans remain elusive. 
This work provided new insight into the differential regulation of cis- and transSNARE associations and suggested the existence of an efficient mechanism that can overcome the intrinsic promiscuity of SNARE interactions and that can contribute to the specificity of intracellular fusion events.

\subsubsection{Tethering factors determine membrane fusion sites and recruit the fusion machinery}

The specificity of intracellular fusion seems to be partially regulated during the step of membrane recognition, termed as docking or tethering. Monitoring the distribution of SNAREs at the interface of docked endosomes, I demonstrated a second mechanism that contributes to the selectivity of SNARE pairing for fusion: the clear exclusion of non-cognate SNAREs and the enrichment of cognate partners at prospective sites of fusion (Figure 3.6). Interestingly, the highly interconnected functions of the docking and fusion machineries were further confirmed when downregulation in the expression of endosomal syntaxins 6 and 13 led to the enhanced recruitment of the Rab5 effector EEA1 on endosomal membranes, and to an increase in endosomal docking activity. Along the same lines, yeast cognate SNAREs accumulate at the interfaces of docked vacuoles, as part of macromolecular lipid-protein complexes, including docking factors as Rab proteins and members of the HOPS complex (Wang et al., 2003). It may therefore be that the docking machinery is involved in the local re-arrangements of SNARE molecules at the fusion interfaces.

\section{Tethers interact with SNAREs}

Factors promoting the initial membrane recognition step have been identified for nearly all intracellular fusion processes (Sztul and Lupashin, 2006), and due to their functional cross-talk with SNARE proteins, they are thought to contribute to the high fidelity of vesicle targeting. There are multiple examples of tether-SNARE interactions. The dual function of the tethering protein and Rab1-effector p115 in intra-Golgi fusion is rather impressive. The protein mediates the tethering of COPI to Golgi membranes by its interaction with Golgins and additionally promotes the assembly of three distinct syntaxin 5-containing SNARE complexes, via its direct 
interaction with GS28 and syntaxin 5 (Shorter et al., 2002). Interestingly, the regulation may actually be bidirectional, since p115 and GM130 seem to require the presence of uncomplexed monomeric syntaxin 5 to exert their tethering function (Bentley et al., 2006). Other multisubunit tethers that associate with SNAREs are the TRAPP complex (transport protein particle), which might tether ER-derived vesicles to the Golgi, the GARP/VFT complex (Golgi associated retrograde protein), with a function in transport between endosomes and the late Golgi and the so called HOPS/class C Vps (homotypic fusion and vacuole protein sorting), which tethers vesicles to late endosomes and the vacuole (Malsam et al., 2008).

\section{The role of EEA1 in the specificity of early endosomal fusion}

Particularly relevant for the regulation of SNARE pairing specificity in homotypic early endosomal fusion is the observation that the tethering, coiled-coil protein EEA1 (early endosomal antigen 1) seems to interact with the early endosomal SNAREs syntaxin 6 (Mills et al., 2001; Simonsen et al., 1999) and syntaxin 13 (McBride et al., 1999). EEA1 is an effector of the early endosomal small GTPase Rab5, associates with $\mathrm{PI}(3) \mathrm{P}$ on the endosomal membrane via its FYVE domain and with Rab5 via domains present in its $\mathrm{C}$ - and N-terminus. As part of the microdomains organized by Rab5 on the endosomal membrane and due to its interaction with endosomal SNAREs, EEA1 would be a candidate regulator of the spatial segregation of cognate versus non-cognate SNAREs that I observed at the docking interface of endosomes (Figure 3.6). Rab5 defines the prospective fusion site and recruits at this position its effectors, including EEA1. Due to its structure that allows simultaneous binding to two membranes (via Rab5 associations), EEA1 subsequently serves as a molecular link, mediates the first recognition step between two endosomes, initiates their contact and accumulates the necessary cognate SNARE molecules via specific interactions.

Interestingly, the oligomeric EEA1-SNARE complexes were also shown to contain the SNARE-pairing-regulatory protein NSF. An attractive hypothesis is that these Rab5-domains include only monomeric, ready-to-fuse SNAREs and NSF ensures their dissociation from pre-assembled cis-SNARE complexes. Since the 
majority of cis-complexes on the endosomal membrane proved to be non-cognate, the co-operative action of EEA1 and NSF may serve to free the endosomal SNAREs from promiscuous interactions and prepare them for meeting their cognate partners in trans. NSF disassembles the cis-complexes, EEA1 interacts with the endosomal SNAREs, stabilizing them at the docking sites, while non-cognate SNAREs can diffuse out and be spatially excluded from the docking and future-fusion interfaces. Thus, indirectly, it is still the SNAREs that control specificity in fusion, but not via their specific associations with other SNARE isoforms, but rather via their crosstalking with regulatory and tethering molecules.

In summary, although the exact molecular interactions governing SNARE pairing specificity are still under investigation, this work provides new aspects on this highly debated issue. Since SNAREs proved to be rather unspecific in their associations, either in vitro as soluble proteins (Fasshauer et al., 1999) or reconstituted on liposomes (Yang et al., 1999) or on native membranes (Bethani et al., 2007), the contribution of additional regulatory molecules to the observed fusion specificity seems mandatory. Even though a certain degree of specificity seems to be encoded in yeast SNAREs (McNew et al., 2000; Scales et al., 2000), the selective complex formation between cognate SNAREs in endosomal fusion observed here is efficiently regulated by mechanisms functioning in a co-operative manner, upstream of fusion.

\subsection{The response of the fusion machinery to SNARE knock- down}

An open question on the specificity governing SNARE interactions and function is whether this highly regulated system can sustain its function upon loss of a certain SNARE isoform. Therefore, I investigated the response of the endosomal function to the elimination of the key SNARE players by siRNA means. Surprisingly, fusion specificity remained largely unaffected while the endosomal system was insensitive to the downregulation of its key components. My results indicate that two complementary mechanisms could account for the observed robustness of the fusion machinery: first, the superabundance of SNARE proteins, that can justify the functionality of 
the fusion machinery even upon a 90\% downregulation in SNARE expression; and second, the tight co-regulation of the fusion and docking components, since knockdown of endosomal SNAREs led to enhanced function of the docking machinery.

As already mentioned in the Introduction, the insensitivity of some intracellular fusion mechanisms to the elimination of SNARE molecules is not a rare observation, but examples in which SNARE knock-downs interfered with the function of certain pathways should not be ignored. Typically, while some trafficking pathways are inhibited, other sorting steps remain unaffected.

Syntaxin 16 and syntaxin 5 knock-downs in HeLa cells blocked the transport of three markers (Shiga toxin, Cholera toxin and ricin) from early endosomes to TGN, whereas transferrin, EGF and VSVG trafficking were not altered, even upon downregulation of syntaxin 16, which has been implicated in endosomal fusion (Amessou et al., 2007). Interestingly, in the same study, the transport of Shiga toxin from TGN to ER was not affected in any of the knock-downs, even though syntaxin 5 was shown to moderate this process in previous studies (Hong, 2005). In a similar study, syntaxin 5 knock-down induced a mild fragmentation of the Golgi, but had no effect on the transport of VSVG across the Golgi and ER to the plasma membrane (Suga et al., 2005). Knock-down of syntaxin 6 in human skin fibroblasts (HSFs) blocked caveolar endocytosis, but did not affect transferrin uptake (Choudhury et al., 2006). SiRNA elimination of syntaxin 10 in HeLa cells resulted in reduced MPRs levels and hypersecretion of hexominidase, indicative of malfunction in MPR trafficking. In the same study, syntaxin 6 knock-down blocked Cholera toxin and TGN46 transport to Golgi (Ganley et al., 2008). In contrast, work from Wang et al. (2005) suggested that syntaxin 10 knock-down did not interfere with the transport of Shiga toxin from early endosomes to TGN, but rather affected the distribution of the TfnR. Finally, syntaxin 6 (Watson et al., 2008), syntaxin 16 (Proctor et al., 2006) and vti1a (Bose et al., 2005) knock-downs seemed to interfere with insulin-stimulated glucose uptake and GLUT4 trafficking in adipocytes.

In all previous studies, the endosome system appears to be strongly resistant to SNARE elimination. In C. elegans, even deletion of both syntaxin 13 and syntaxin 16 had no impact on endocytosis and endosome size (Gengyo-Ando et al., 2007). Even 
complex organelles such as the Golgi appear to suffer little from lacking certain SNAREs, since only knock-down of syntaxin 5 slightly affected Golgi morphology (Suga et al., 2005). Similarly, there are even cases of knock-out mice that exhibited no severe phenotypes despite the total elimination of a SNARE molecule. Knock-out mice for the late endosomal SNARE vtilb were viable and fertile, with no severe effects on lysosomal transport and function (Atlashkin et al., 2003). Elimination of VAMP8 did not affect the mice development and fertility and had only an effect on pancreatic cells (Wang et al., 2004), while mice lacking VAMP3 had normal growth and displayed normal endocytosis or general constitutive membrane recycling (Yang et al., 2001).

\subsubsection{SNAREs are abundant and organize in membrane clusters}

\section{SNARE abundance explains the insensitivity of endosomal function to SNARE downregulation}

The absence of severe phenotype upon the elimination of a specific SNARE can be explained by two different scenarios. First, the specificity in SNARE function may not be as strict as envisioned, with related SNAREs functionally replacing the missing protein. While this is clearly the case upon SNARE knock-out, an alternative possibility explaining the lack of phenotype upon SNARE downregulation is that the members of the SNARE family are expressed in high levels that exceed the cellular needs. Therefore, even a 90\% downregulation in SNARE expression can be tolerated by the fusion machinery. Interestingly, the concept of super-abundance is not unique for SNARE proteins, since acetylcholine receptors (AchRs) also seem to be provided in excess. In myastenia gravis, a disease related to the reduced number of available AchRs, patients reveal symptoms of muscle malfunction only after the levels of the receptors are reduced by 70\% (Myasthenia Gravis Fact Sheet, National Institute of Neurological Disorders and Stroke, NIH). Nevertheless, as discussed in the previous section, some intracellular trafficking pathways are indeed malfunctioning upon SNARE knock-down. A simple interpretation of these seemingly controversial phenotypes is that the SNAREs participate selectively in certain pathways in each of the investigated systems. However, it is known that these SNAREs are not selec- 
tively localized on certain subtypes of endosomes (with, for example, syntaxin 6 and VAMP4 being even abundantly expressed in the Golgi system (Advani et al., 1998; Wendler and Tooze, 2001)). In addition, they have already been demonstrated to function in the fusion of non-specifically labeled endosomes (Brandhorst et al. (2006); Rizzoli et al. (2006)), which implies their activity is necessary for a variety of endosome types. Therefore, it can be suggested that the SNARE abundance allows the organelles housing these molecules to function as long as certain critical levels are not reached. The phenotypes noted above would presumably appear when the SNARE needs are too high (either temporally or spatially) to be fulfilled by the residual pool.

Are SNARE proteins indeed so abundant as to ignore massive knock-downs? Syntaxin 1 and SNAP-25 are highly expressed in neuronal cells, each constituting around $4 \%$ of the total synaptosomal protein (Walch-Solimena et al., 1995). Similarly, each synaptic vesicle contains 70 copies of synaptobrevin, in contrast to 15 and 30 copies, respectively, of the two most abundant regulatory proteins, synaptotagmin and synaptophysin (Takamori et al., 2006). This abundance of SNARE proteins is even more striking when one considers the number of SNARE complexes that are necessary for fusion, which has been estimated to be between 3 and 15 (Montecucco et al., 2005). This fact can additionally explain why fusion persists when the number of available SNAREs is severely decreased in the comatose fly mutant that has a NSF malfunction (Kawasaki et al., 1998). Even though this model of SNARE abundance can satisfactorily explain the lack of phenotype in knock-down approaches, it cannot apply to SNARE knock-outs, where only SNARE redundancy can act. Mice lacking synaptobrevin 2 die immediately after birth and exhibit a 100-fold decrease in fast $\mathrm{Ca}^{2+}$-triggered exocytosis in hippocampal neurons (Schoch et al., 2001). However, loss of synaptobrevin in chromaffin cells does not impair exocytosis. Only when both synaptobrevin and cellubrevin are eliminated, secretion is abolished in chromaffin cells, suggesting the complementary function of these proteins (Borisovska et al., 2005). Similar observations on functional redundancy between VAMPs have been also reported in Drosophila by Bhattacharya et al. (2002). Examples of incomplete compensation by related SNAREs also include SNAP-25 that can be partially sub- 
stituted by exogenously expressed SNAP-23 in chromaffin cells (Delgado-Martnez et al., 2007), and the yeast SNAREs Sec22 (partial rescue by Ykt6 (Liu and Barlowe, 2002)), or Pep12p (partial rescue by Vam3p (Darsow et al., 1997)). Finally, redundancy can arise from recent gene duplication events and the existence of isoforms with identical function for some SNAREs such as syntaxin 1 (Bennett et al., 1993), synaptobrevin (Elferink et al., 1989), Snc (Protopopov et al., 1993) and Sso (Aalto et al., 1993).

Nevertheless, none of the proteins that substitute for the absence of related SNAREs seems to elevate its expression level, indicating that their initial amount can satisfy both their actual and their compensatory role. Thus, it can be suggested that SNARE abundance is the key mechanism ensuring the robustness of the fusion machinery against stress conditions, either due to the sufficiency of the residual protein levels or to the overtaking SNARE isoforms.

\section{Characteristics and role of SNARE clusters on native membranes}

Investigating SNARE interactions in knock-down organelles, I observed that the residual SNARE levels participate to a comparable amount of complexes as detected for the wild type situation. This observation posed the question of how the abundant SNARE molecules organize on membranes and escape the participation in cis-SNARE interactions. In accordance to previous studies, immunostaining and microscopic experiments revealed the presence of homooligomeric SNARE clusters on the membrane of early endosomes (see Figure 3.19). Studies on inverted membrane lawns (Lang et al., 2001; Predescu et al., 2005; Sieber et al., 2006) and intact cells (Aoyagi et al., 2005; Rickman et al., 2004) showed that SNAREs, mediating regulated and constitutive exocytosis, form distinct microdomains on the plasma membrane of various cell types. Biochemical studies further suggested that the three exocytic SNAREs are associated with lipid rafts and cholesterol depletion disturbs the clustering and inhibits exocytosis (Chamberlain et al., 2001). These data are in accordance with my observations, where cholesterol depletion led to synaptobrevin un-clustering and increased its SNARE associations (see Figure 3.20). The role of cholesterol in the stability of clusters and indirectly in exocytosis has been appreci- 
ated by several studies (Chamberlain et al., 2001; Lang et al., 2001; Predescu et al., 2005), but one should not underestimate the role of homomeric SNARE-SNARE interactions in the cluster formation and stability (Sieber et al., 2006, 2007).

The exact biological function of SNARE microdomain organization is still unclear. It is believed that these clusters spatially organize docking and fusion sites on membranes, due to the correlation between unclustering and inhibition of exocytosis upon cholesterol depletion, as well as to the colocalization of secretory vesicles with membrane SNARE domains (Lang et al., 2001; Predescu et al., 2005). The high accumulation of homomeric SNAREs in specific membrane spots may increase the efficiency of trans-SNARE pairing and contribute to the speed of the fusion reaction.

Alternatively, SNARE oligomers may also serve as reserve, storage pools. In accordance to this idea, Sieber et al. (2006) showed that the number of syntaxin 1 clusters on the membrane is proportional to the concentration of the particular SNARE, indicating that microdomain organization may serve as a mechanism to store excess protein. Additionally, when the cellular demand is high, SNAREs could be retrieved easily from such homooligomers, circumventing the need of large amounts of energy that would be required to dissociate them from possible heterooligomeric SNARE complexes via NSF function. Furthermore, my results indicate that in the case of SNARE downregulation, when the amount of expressed protein is severely reduced, the residual protein loses its domain organization on the endosomal membrane and exhibits a more diffused pattern, in order to accommodate the cellular needs (see Figure 3.19). Therefore, the cluster organization of SNARE molecules on membranes may serve as a storing mechanism of the excess protein that is expressed. Along the same lines, cluster formation may also contribute to the specificity of membrane fusion by reducing the number of non-cognate unproductive cis-SNARE complexes that are present on the endosomal membrane (see Introduction). Since mass-action is determining the amount and identity of SNARE associations in cis, the organization of SNAREs in membrane microdomains would decrease the number of molecules available for interactions and consequently, the probability of their engagement in non-cognate associations. 


\subsubsection{Co-operative functions of the docking and fusion machinery}

Because of the tight co-operation between the docking and fusion machinery and the contribution of tethering factors to the specificity of intracellular fusion, the enhanced docking activity observed upon SNARE knock-down was not surprising. Comparing the distribution of EEA1 between membrane and cytosol in wild type and knock-down cells, I showed that SNARE elimination results in the enhanced recruitment of EEA1 on the endosomal membrane. Additionally, application of in vitro docking techniques revealed enhanced docking of early endosomes from cells knocked-down for the endosomal SNAREs.

These observations are in accordance with previous studies reporting increased docking upon SNARE elimination. Cleavage of syntaxin 1 caused the accumulation of docked vesicles at the squid giant synapse (Marsal et al., 1997). Although the accumulation of docked synaptic vesicles is probably a side-effect of their inability to undergo fusion in absence of syntaxin 1, the increased docking observed between early endosomes is most likely a facilitation mechanism that promotes fusion under the stress conditions of endosomal SNARE downregulation. Despite the catalytic role of SNARE proteins in membrane fusion, enhanced activity of the docking machinery can independently lead to enhanced fusion, suggesting that tethering/docking are the rate-limiting steps in fusion. For example, the overexpression of the GTPases Rab5 and Rab9 leads to enhanced endosomal fusion and the formation of enlarged early and late endosomes, respectively (Riederer et al., 1994; Stenmark et al., 1994), even though these proteins act upstream of the fusion machinery.

EEA1 is part of the Rab5-organized, macromolecular complexes that mediate tethering of early endosomes. It exhibits a long, coiled-coil structure and it possesses an N-terminal $\mathrm{C}_{2} \mathrm{H}_{2}$, Rab5-binding domain, four heptad repeats and a C-terminal region that contains a calmodulin binding (IQ) motif, a Rab5-interacting region and a FYVE domain, that allows its association to PI(3)Ps. Its association with syntaxins 13 and 6, as well as with the fusion regulatory ATPase NSF indicates the potential role of EEA1 in the regulation of specificity in endosomal fusion. As already discussed, the combinatorial function of EEA1 and NSF might promote the accumulation of the cognate SNAREs at the fusion sites. But why does the 
downregulation of early endosomal SNAREs increase the amount of EEA1 on the endosomal membrane?

As demonstrated by McBride et al. (1999), the interaction between EEA1 and syntaxin 13 seems to be a prerequisite for fusion. Overexpression of an EEA1fragment that competes with the endogenous protein for binding to syntaxin 13 completely abolished fusion and even overshadowed the effect of Rab5Q79L. In addition, excess of recombinant EEA1 was shown to overcome the requirement for cytosol in endosomal fusion in vitro, demonstrating the catalytic contribution of this tethering molecule to endosomal fusion (Christoforidis et al., 1999). Therefore, the increase in EEA1 domains on the endosomal membrane upon syntaxin 13 knockdown may function as a compensatory mechanism that sustains endosomal fusion. According to the affinity of the EEA1-syntaxin 13 interaction, the SNARE seems to be only transiently incorporated in the EEA1 clusters, so that increased levels of EEA1 would enhance the chances of the residual syntaxin 13 to get recruited in the EEA1-containing-domains. In parallel, the abundance of EEA1 stimulates endosomal docking, removing a limiting step from the already stressed fusion machinery.

It has been shown that the assembly of Rab effectors in membrane domains is highly dynamic with the oligomeric clusters appearing heterogeneous in size rather than as stable and defined complexes, due to the constant cycling of Rab5 between its GTP-membrane-bound and GDP-cytosolic state (Rybin et al., 1996). In addition, the cellular EEA1 reservoir seems to consist of two pools, one rapidly exchanging between the cytosol and the endosomal membrane and one with longer membrane residence (Bergeland et al., 2008). The potential contribution of NSF to the stability of these complexes is of special interest. EEA1 and Rabaptin-5 are stabilized in presence of the NSF-inhibitor $\mathrm{ATP} \gamma \mathrm{S}$ and are released from the NSF oligomers when the co-factor $\alpha$-SNAP is present (McBride et al., 1999). These results also explain the absence of $\alpha$-SNAP from the EEA1-oligomers, since in its presence NSF can exhibit its function and de-stabilize the complex.

Taking together the dynamic properties of the EEA1-clusters and the role of NSF in the regulation of their stability, it can be suggested that SNAREs may also contribute to the life-time of these oligomers. The incorporation of SNARE pro- 
teins and $\alpha$-SNAP to the membrane clusters may trigger the activation of NSF and the subsequent domain de-stabilization. Upon siRNA downregulation of endosomal SNAREs, the low levels of SNARE molecules present on the membrane decrease the chances of SNARE participation in EEA1 domains, NSF remains inactive (in absence of substrate) and the lifetime of clusters increases, with more EEA1 being detected on the membrane.

\subsection{Conclusions}

Addressing the controversial issue of regulation in SNARE function specificity, this work shed new light on the properties of the fusion machinery as well as on its relation and co-operation with upstream mechanisms. The selectivity for cognate SNARE interactions in trans and the local concentration of cognate SNAREs in fusion sites provided evidence for the existence of proof-reading mechanisms in SNARE pairing that specifically function in trans and suggested the involvement of the docking machinery in this regulatory process. Interestingly, downregulation of SNARE expression confirmed the tight connection of the docking and fusion machineries, since an increased membrane recruitment of docking factors and enhanced docking activity were observed upon SNARE loss.

Although the exact molecular players mediating the regulation of fusion specificity and the specific mechanisms of co-operation between docking and tethering factors are still unknown, it can be appreciated from this work that the coordination of the essential process of membrane fusion is the result of the highly co-operative function of multiple players. As an example of coincidence detection (Paolo and Camilli, 2006) on a macromolecular level, the mechanisms controlling the specificity and robustness of the fusion machinery can only be efficient via their combinatorial action. Only the co-operation of SNARE pairing selectivity in trans with the lateral segregation of SNAREs ensures specific and productive fusion, while the super-availability of SNAREs together with the tight connections between fusion and docking factors provide robustness to intracellular trafficking. 


\section{5 \\ SUMMARY}

Intracellular transport is a vital cellular process that ensures cell homeostasis and survival. It involves the exchange of material among different organelles and it is mediated by the packaging of cargo in transport vesicles, which bud form the compartment of origin and fuse with the target membrane. SNARE (Soluble Nethylmaleimide-sensitive factor attachment receptor) proteins are the key regulators of membrane fusion. SNAREs present in two opposing membranes associate in complex, thereby bringing the membranes in proximity and facilitating lipid mixing. Different SNARE molecules are believed to function in fusion events that involve different processes and organelles. However, it is still unclear how cognate SNAREs specifically interact with each other for fusion, since the continuous membrane mixing occurring during intracellular transport results in a redistribution of SNARE molecules in compartments where they do not exhibit their function.

This question becomes particularly relevant for the early endosomal compartment, which is positioned in the center of the endocytic pathway and is constantly exchanging material with a variety of organelles. Therefore, it contains not only SNAREs mediating endosomal fusion, but also additional sets of SNAREs that are 'passengers' en route to their resident compartment, including those mediating exocytosis and fusion of late endosomes.

During my Master's thesis, I had shown that early endosomal and exocytic SNAREs can associate promiscuously on the endosomal membrane, even though their functions do not overlap, with the first SNARE set mediating homotypic early endosomal fusion and the later set functioning in exocytosis. My PhD work focused on the question of regulation in specificity of SNARE pairing and function. To this end, I applied a variety of biochemical and microscopic techniques using the early 
endosomal and exocytic SNAREs from PC12 cells as my experimental platform. I found that the distributions of exocytic and endosomal SNAREs spatially overlap on the endosomal membrane and that cis-SNARE interactions are mainly governed by mass-action. However, I showed that the function of two co-operative mechanisms ensures SNARE-pairing specificity upon fusion. First, SNARE molecules associating in trans-configuration show a preference for cognate interactions. Second, SNAREs are laterally segregated, with cognate isoforms enriching at fusion sites and noncognate ones being excluded, suggesting the existence of regulatory steps during the docking process.

Nevertheless, questions remained regarding the robustness of such highlyregulated system. Is specificity preserved when the fusion machinery is challenged, for example by low availability of its cognate SNARE molecules? Thus, I downregulated the expression of early endosomal and exocytic SNAREs in PC12 cells using siRNA. By application of various cellular and biochemical assays, I found no effect in cell viability, endosomal fusion and intracellular trafficking upon SNARE knock-down. However, two observations suggested the existence of compensatory mechanisms that would account for the absence of severe phenotypes. SNARESNARE interactions were not altered in knock-down cells, with the residual SNARE protein levels participating in a number of interactions similar to wild type. Studying the distribution of SNAREs on the endosomal membrane, I observed that the proteins are organized in clusters that might serve as reserve pools of molecules storing the excess protein and preventing it from participating in SNARE-SNARE interactions. Additionally, knock-down of early endosomal SNAREs resulted in the increased recruitment of the tethering factor EEA1 on the endosomes and enhanced docking activity, indicating a tight interconnectivity of the docking and fusion machinery. I therefore conclude that the superabundance of SNARE proteins and the co-regulation of docking and fusion (i) ensure the impeccable function of intracellular fusion, even under stress conditions, (ii) strongly demonstrate the role of docking in the specificity of SNARE-mediated fusion and (iii) stress the importance of the cooperative function of several mechanisms for the maintenance of intracellular trafficking and cell viability. 


\section{A}

\section{APPENDix}

\section{A.1 Monte Carlo simulation of SNARE interactions on the endosomal membrane}

The following program was written using the Matlab programming language (The Mathworks Inc., Natick, MA, USA). It was used to simulate the cognate and noncognate interactions between endosomal and exocytic SNAREs on the endosomal membrane under different conditions. The results are presented in Figures 3.5, 3.7 and 3.18 and the parameters of the program were modified accordingly in each case. The stoichiometries in which the SNARE molecules were introduced, were derived from Table 3.1 and the size of an individual SNARE molecule was approximated from the size of synaptobrevin, as depicted in the model proposed by Takamori et al. (2006). Comments explaining every programming step are shown in green and indicated by the symbol $\%$.

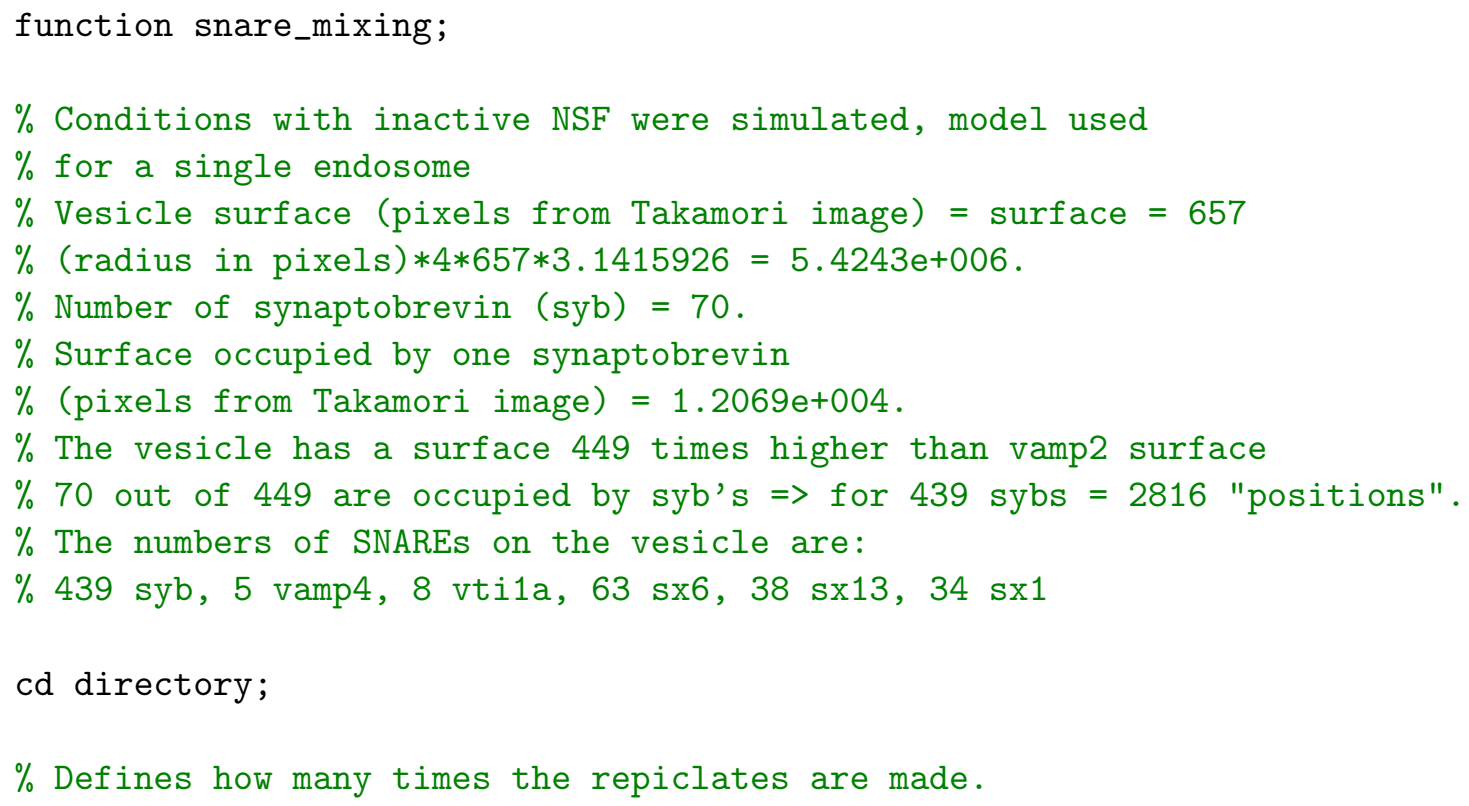


for abcde $=1: 100$

$\%$ Defines the number of SNAREs on the endosome.

qabc $=\operatorname{zeros}(2816,1) ; \operatorname{qabc}(1: 8)=1 ;$

vamp4 $=\operatorname{zeros}(2816,1) ; \operatorname{vamp} 4(1: 5)=1 ;$

$\operatorname{syb}=\operatorname{zeros}(2816,1) ; \operatorname{syb}(1: 439)=1 ;$

qabc2 $=\operatorname{zeros}(2816,1) ; \operatorname{qabc} 2(1: 34)=1 ;$

comp_cog_v = [] ;

comp_noncog_v $=[]$;

comp_cog_syb = [];

comp_noncog_syb $=[]$;

$\%$ Defines the probability that a complex forms when

$\%$ two non-cognate entities meet.

proba $=[]$;

$\%$ The loop that changes the probability is introduced.

for $\mathrm{klm}=1: 1000$

$\%$ qabc=endosomal Q-SNARE complex,

$\%$ qabc2=exocytic Q-SNARE complex

qabc $=\operatorname{zeros}(2816,1) ; \operatorname{qabc}(1: 8)=1 ;$

vamp4 $=\operatorname{zeros}(2816,1) ; \operatorname{vamp} 4(1: 5)=1 ;$

$\operatorname{syb}=\operatorname{zeros}(2816,1) ; \operatorname{syb}(1: 439)=1$;

qabc2 $=\operatorname{zeros}(2816,1) ; \operatorname{qabc2}(1: 34)=1 ;$

$\mathrm{klm}$

$\%$ A matrix with all ones is made- so that any randomly

$\%$ taken sample from it is 1.

prob_cog=[ $\left.\begin{array}{lllll}1 & 1 & 1 & 1 & 1\end{array}\right]$;

$\%$ A matrix with one element being 1 , and the rest 0 ;

$\%$ the size is $=\mathrm{klm}$, which runs from 1 to 1000 .

prob_noncog=zeros $(\mathrm{klm}, 1)$;

prob_noncog $(1)=1$;

$\%$ The number of "available" (for complexing) molecules

$\%$ for each of the four entities.

qabc_er $=8$;

vamp4_er $=5$;

syb_er $=439$;

qabc2_er $=34$;

$\%$ The numbers of complexes formed for each of the four entities.

$\begin{array}{ll}\text { sum_v4_cog } & =[] ; \\ \text { sum_v4_noncog } & =[] ; \\ \text { sum_syb_cog } & =[] ; \\ \text { sum_syb_noncog } & =[] ;\end{array}$

$\%$ The iteration loop starts. The molecules interact 1000 times.

$\%$ Complexes are counted every time and the numbers of available 
$\%$ complexes are changed at each step, as new complexes form.

$\%$ One number of complexes (for each cognate/non-cognate) is

$\%$ given after 1000 iterations - it corresponds to one probability

$\%$ value. In some respect, this is equivalent to incubating endosomes

$\%$ for 1000 seconds, and monitoring how many complexes form.

for $i=1: 1000$

$\%$ The positions of the elements on the endosome are defined.

comqabc = randsample (qabc, 2816);

comvamp4 = randsample (vamp4, 2816);

comsyb = randsample (syb, 2816);

comqabc2 $=$ randsample $(q a b c 2,2816)$;

$\%$ Checking for elements occupying the same place on the

$\%$ endosome. Check does not apply to syb - it is

$\%$ assumed that when syb and $\mathrm{v} 4$ occupy the same

$\%$ place with endosomal qabc, v4 prevails.

$c c c=$ find $($ comqabc $==1$ \& comvamp $4==1)$;

if numel (ccc) $>0$

$\%$ The sum of complexes formed.

ssum_v4 = [];

for $j=1$ :numel (ccc)

$\% 1=$ one complex $*$ probability ; except

$\%$ that here probability is always one.

$\operatorname{ssum}_{-} \mathrm{v} 4(j)=1 *$ randsample $($ prob_cog, 1$)$;

end;

$\%$ The sum of the number of ACTUAL complexes,

$\%$ after multiplication with probability.

sum_v4_cog $(i)=\operatorname{sum}\left(\right.$ ssum_v$\left._{-}\right)$;

else

sum_v4_cog $(i)=0$;

end;

$\%$ The routine checks for vamp4 - sx1/SNAP-25 complexes;

$\%$ if syb is there, it will prevail, so the routine makes

$\%$ sure that syb is excluded.

$c c c=$ find (comqabc2 $==1$ \& comvamp $4==1$ \& comsyb==0);

if numel (ccc) $>0$

ssum_v4 $=[]$;

for $j=1$ :numel (ccc)

$\%$ This is the changing, non-cognate probability. $\operatorname{ssum}_{-} 4(j)=1 *$ randsample $($ prob_noncog, 1$)$;

end;

sum_v4_noncog $(i)=\operatorname{sum}\left(\operatorname{ssum} \_v 4\right) ;$

else

sum_v4_noncog $(i)=0$;

end;

$c c c=$ find $(\operatorname{comqabc} 2==1$ \& comsyb $==1)$;

if numel (ccc) $>0$ 


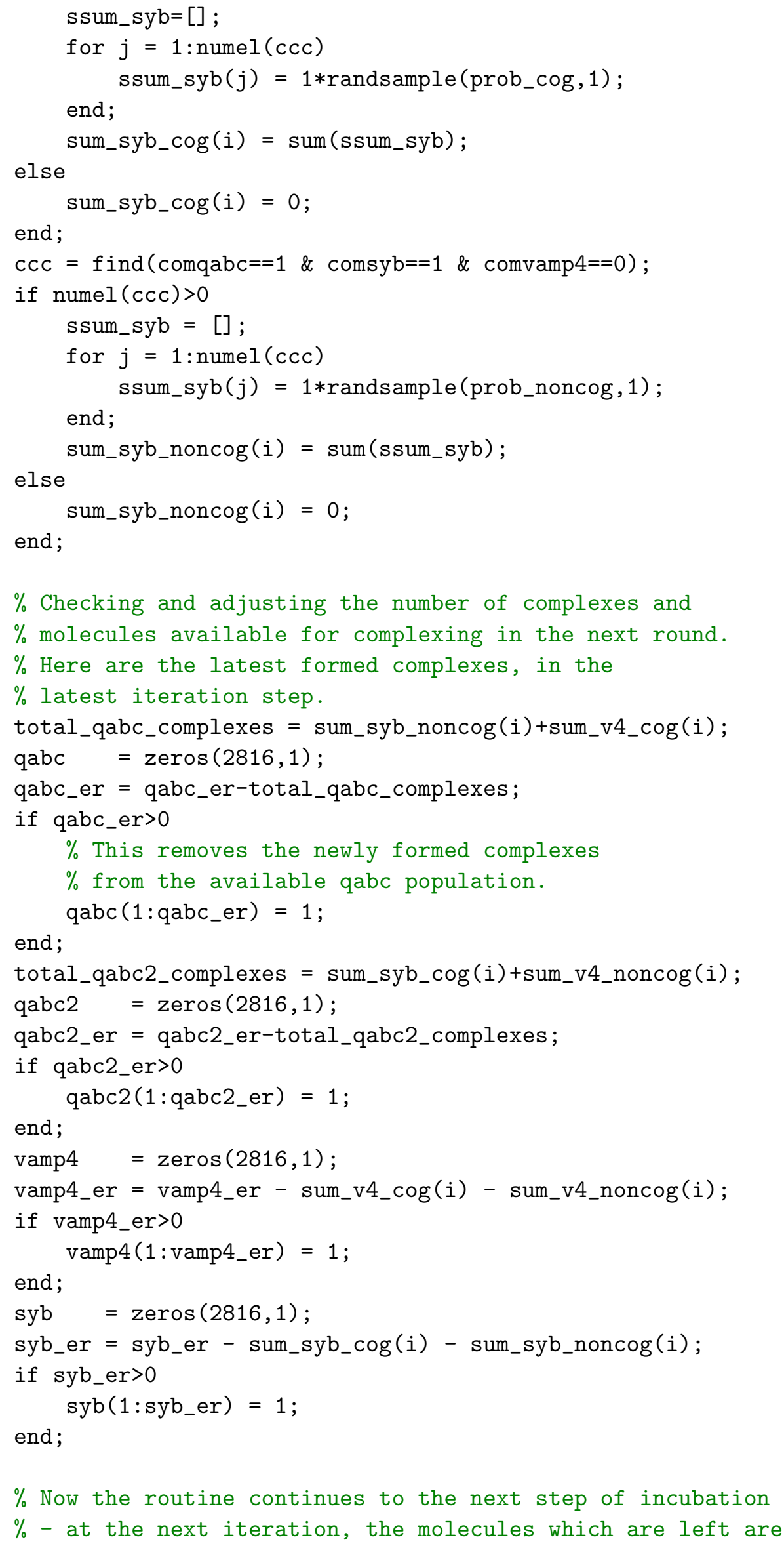


$\%$ allowed to interact again and again, for 1000 times end;

$\%$ The incubation (1000-iteration, using the " $i$ " as counter

$\%$ - the for i loop) is over, complexes can be counted and

$\%$ stored for the particular probability ( $\mathrm{klm}$ ).

comp_cog_v(klm)= sum (sum_v4_cog);

comp_noncog_v $(\mathrm{klm})=\operatorname{sum}\left(\mathrm{sum} \_v 4 \_n o n c o g\right)$;

comp_cog_syb(klm)= sum (sum_syb_cog);

comp_noncog_syb $(\mathrm{klm})=$ sum (sum_syb_noncog);

$\operatorname{proba}(\mathrm{klm}) \quad$ = sum (prob_noncog) $/$ numel (prob_noncog);

end;

$\operatorname{matrix}(:, 1)=$ proba';

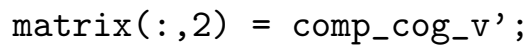

$\operatorname{matrix}(:, 3)=$ comp_noncog_v';

$\operatorname{matrix}(:, 4)=$ comp_cog_syb';

matrix $(:, 5)$ = comp_noncog_syb';

dlmwrite (strcat (num2str(abcde), '_cis_matrix.txt'), matrix);

end; 


\section{A.2 Calculation of the number of fused and docked or- ganelles}

The following routine was written in Matlab (The Mathworks Inc., Natick, MA, USA) and was used to calculate the number of fused and docked organelles in the in vitro fusion assay (described in 2.2.7). Comments explaining every programming step are shown in green and indicated by the symbol $\%$.

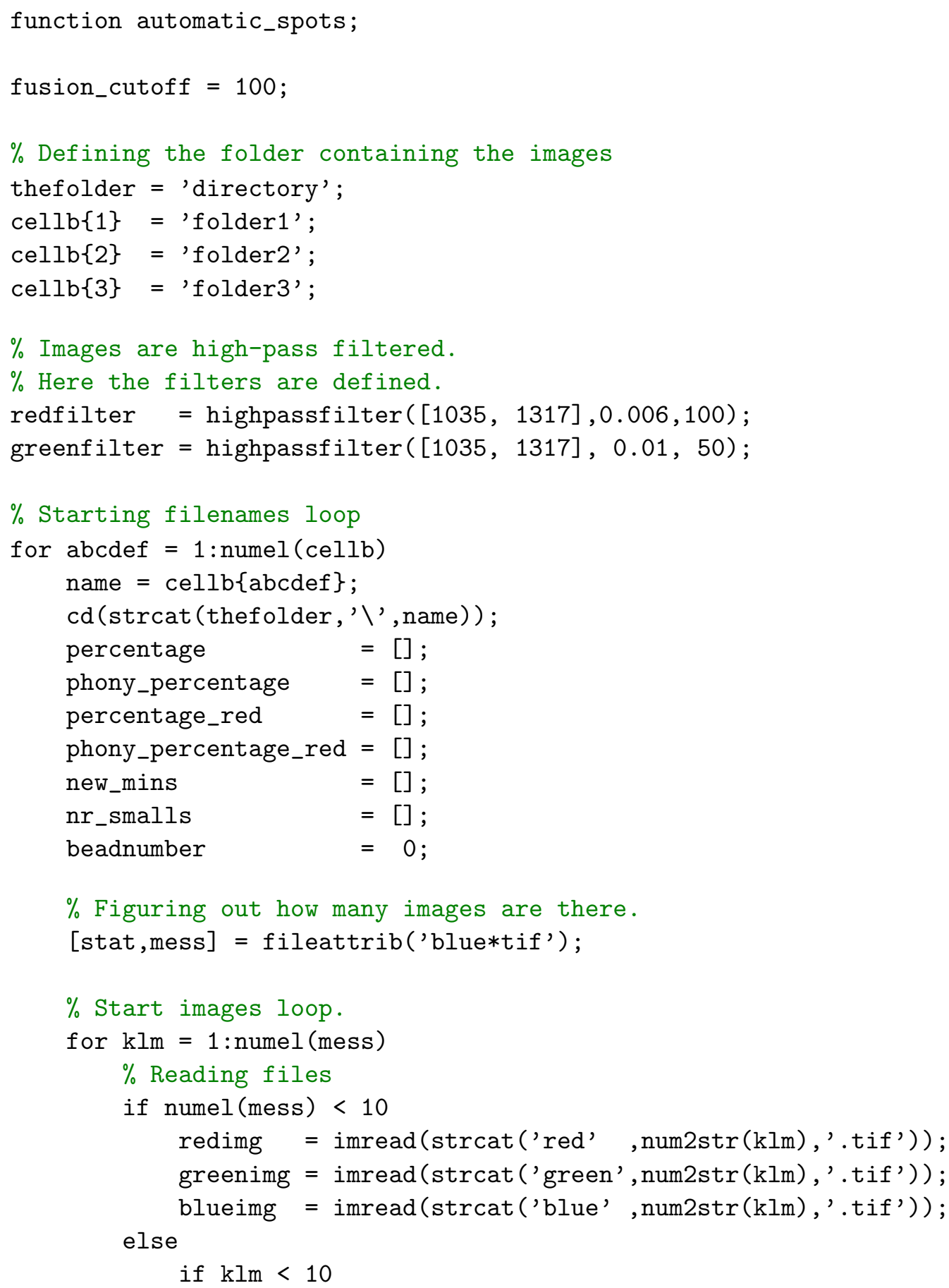




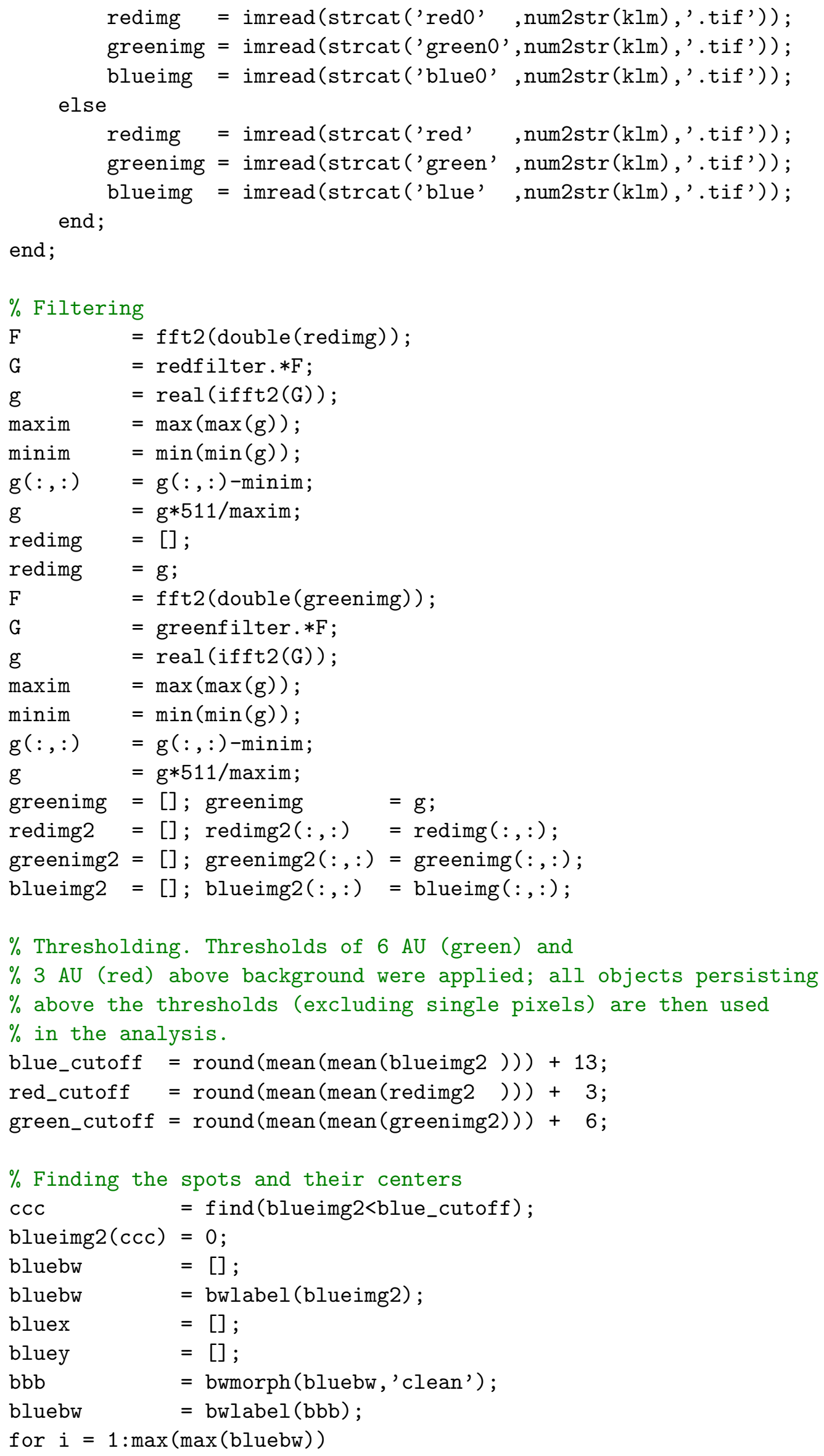




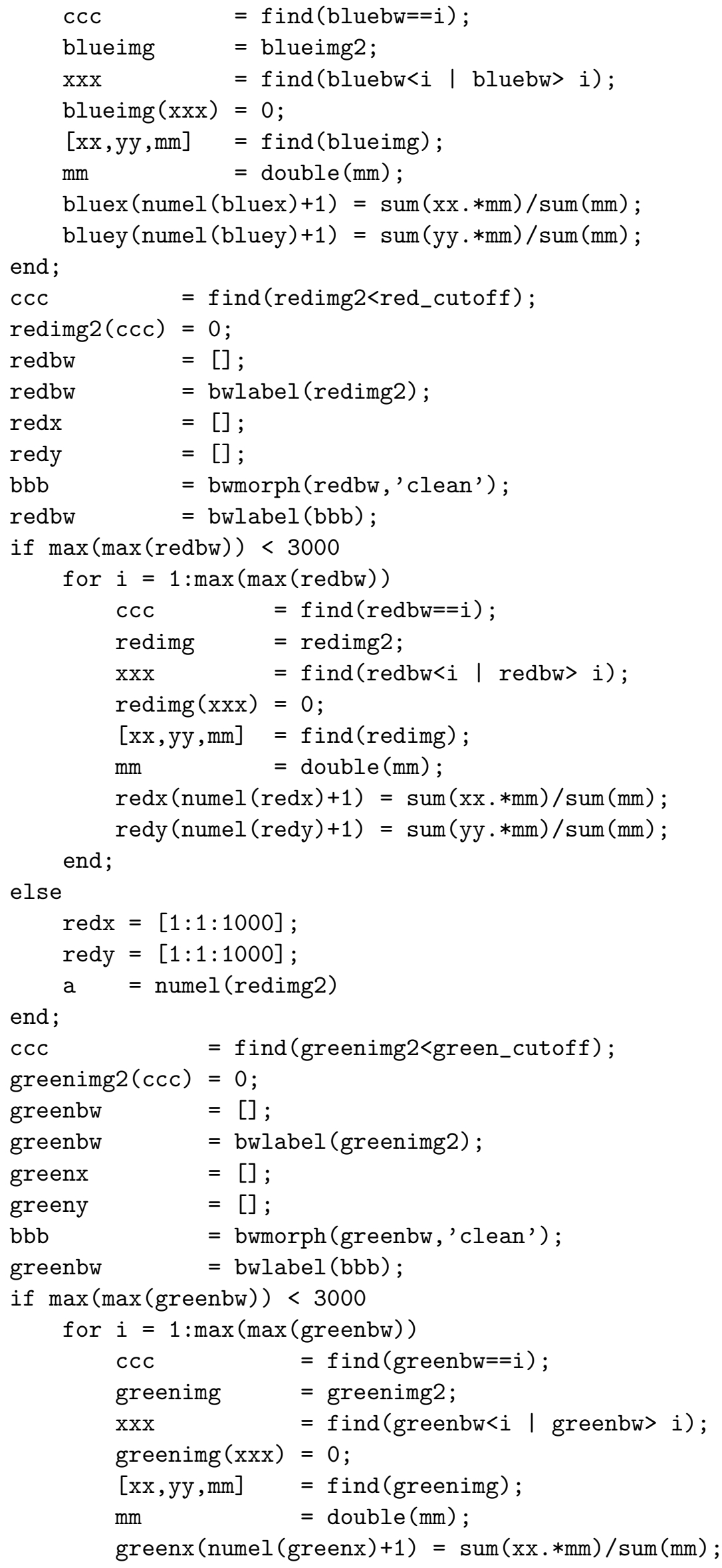




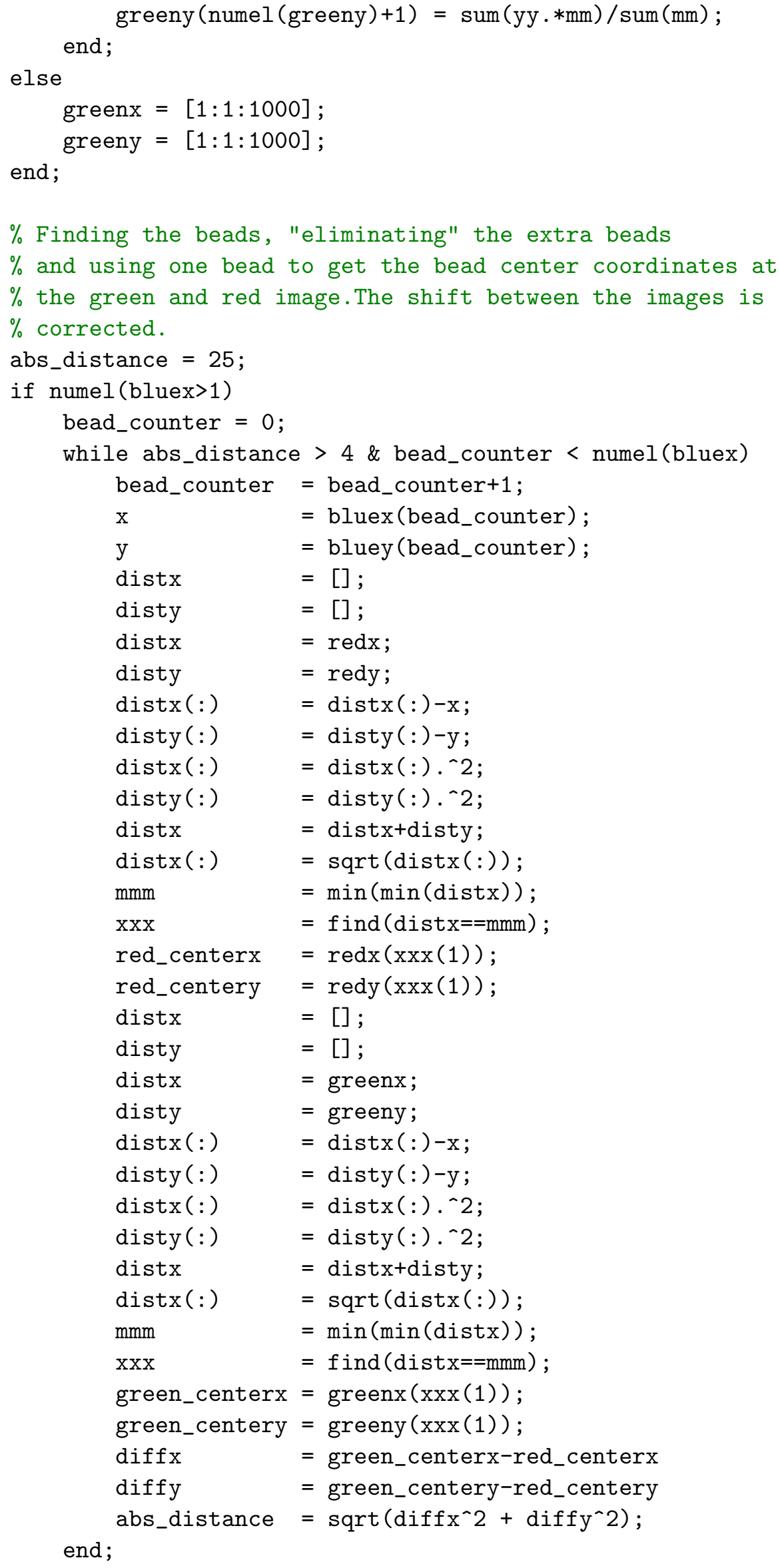




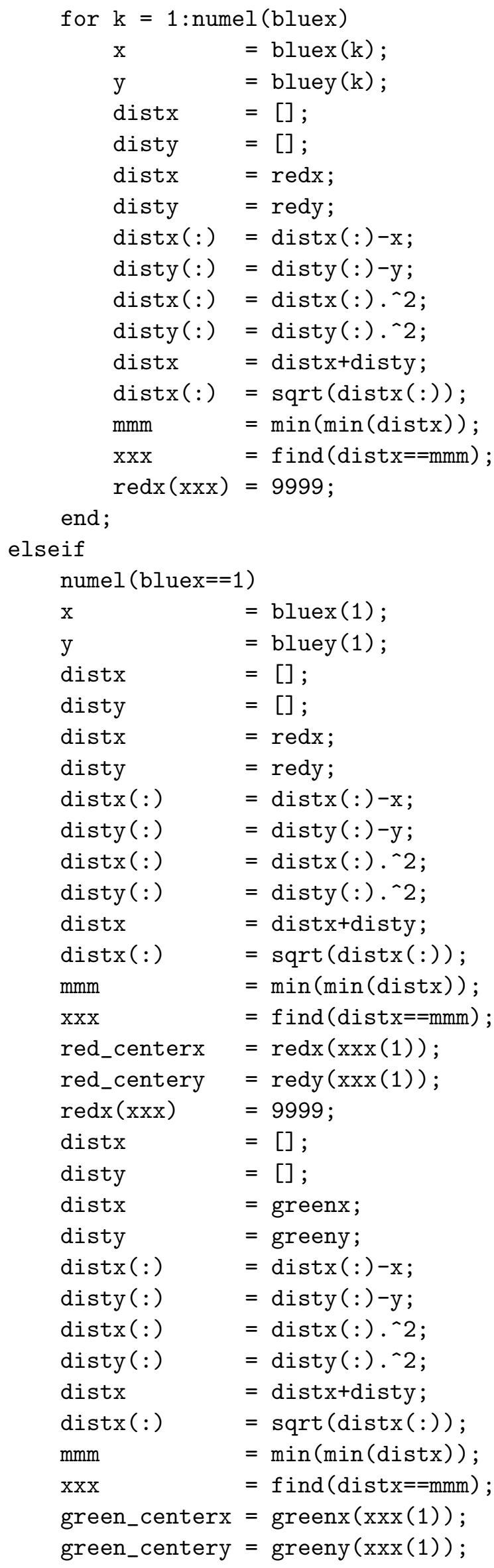




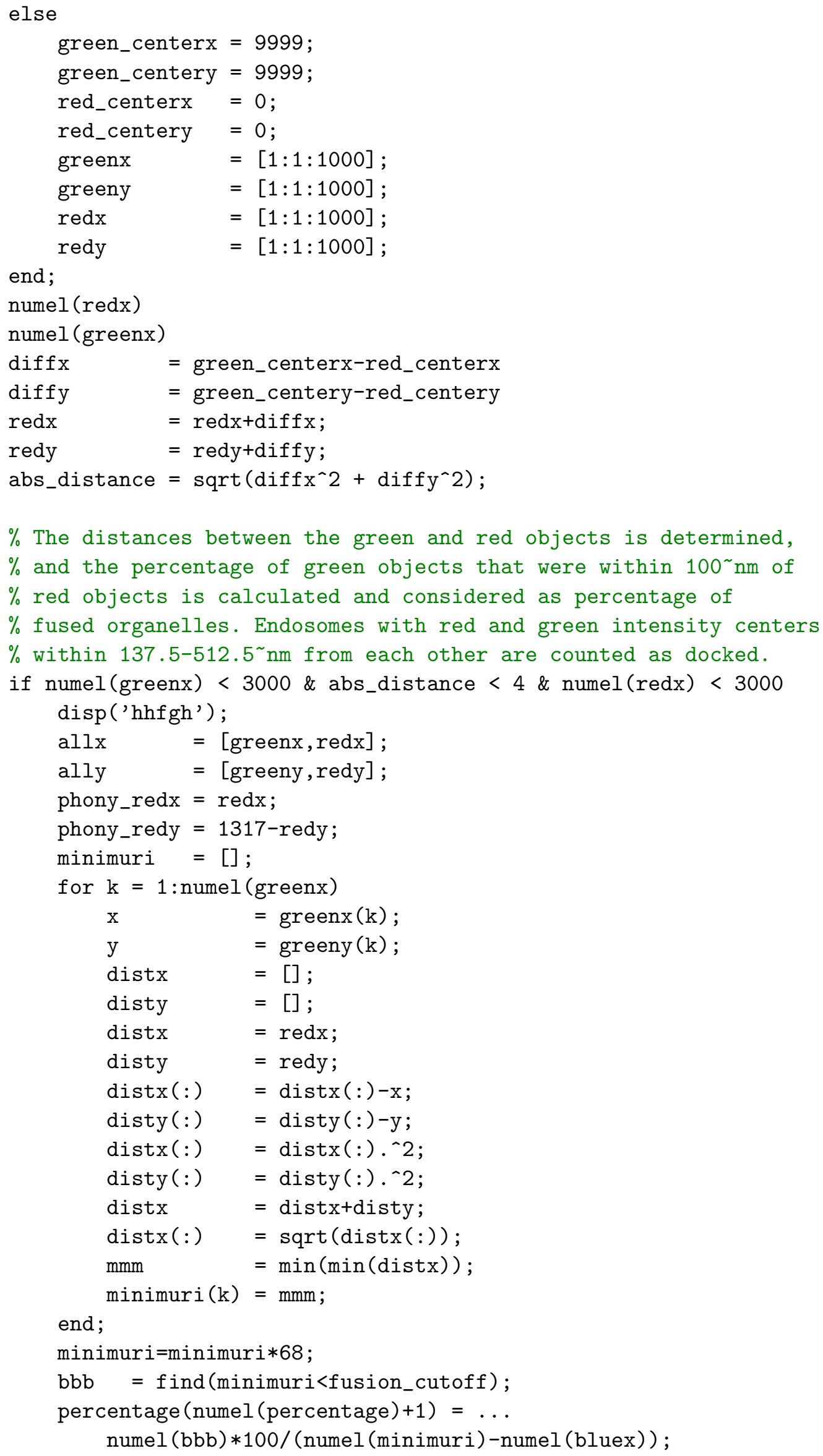




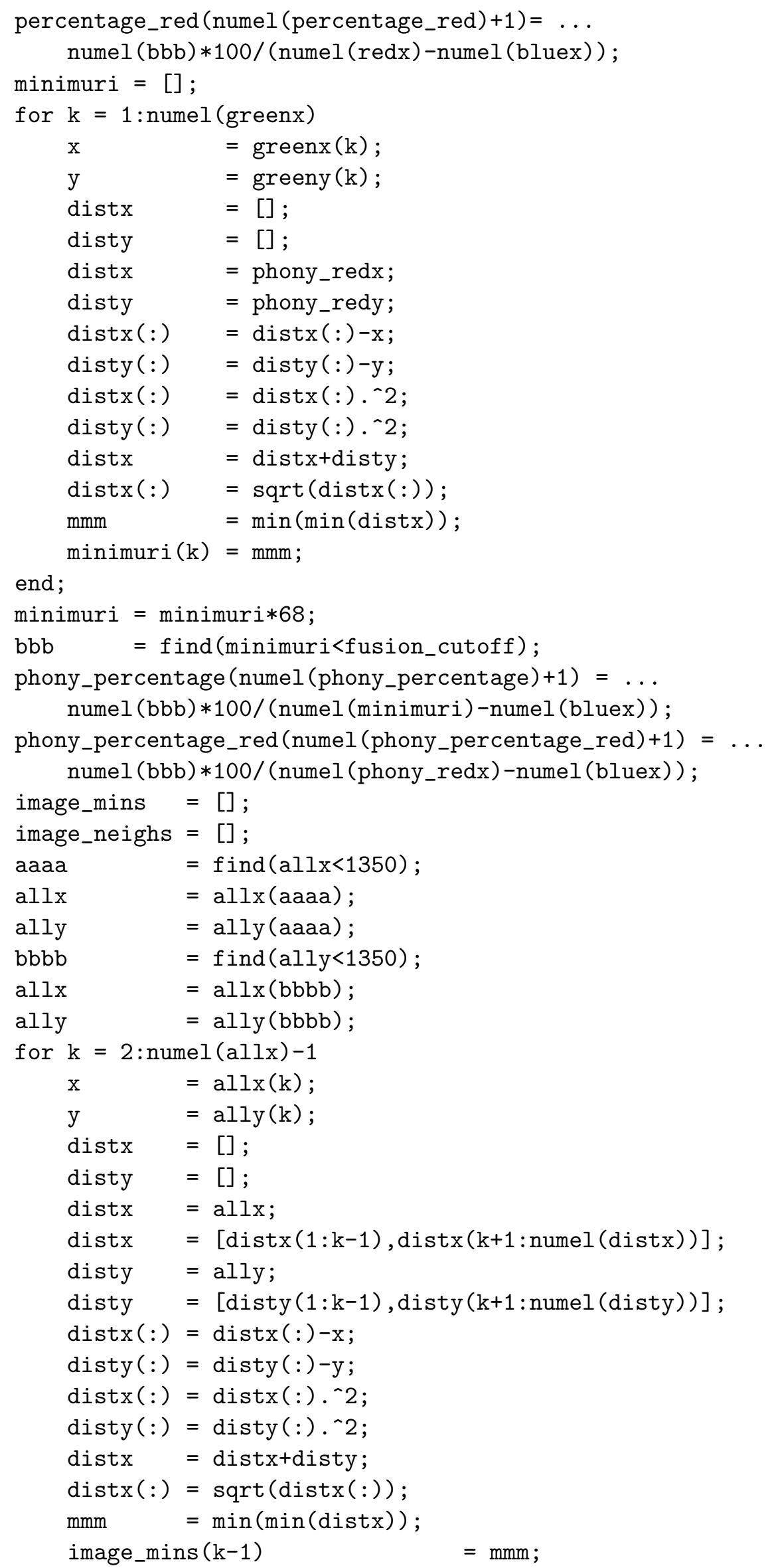




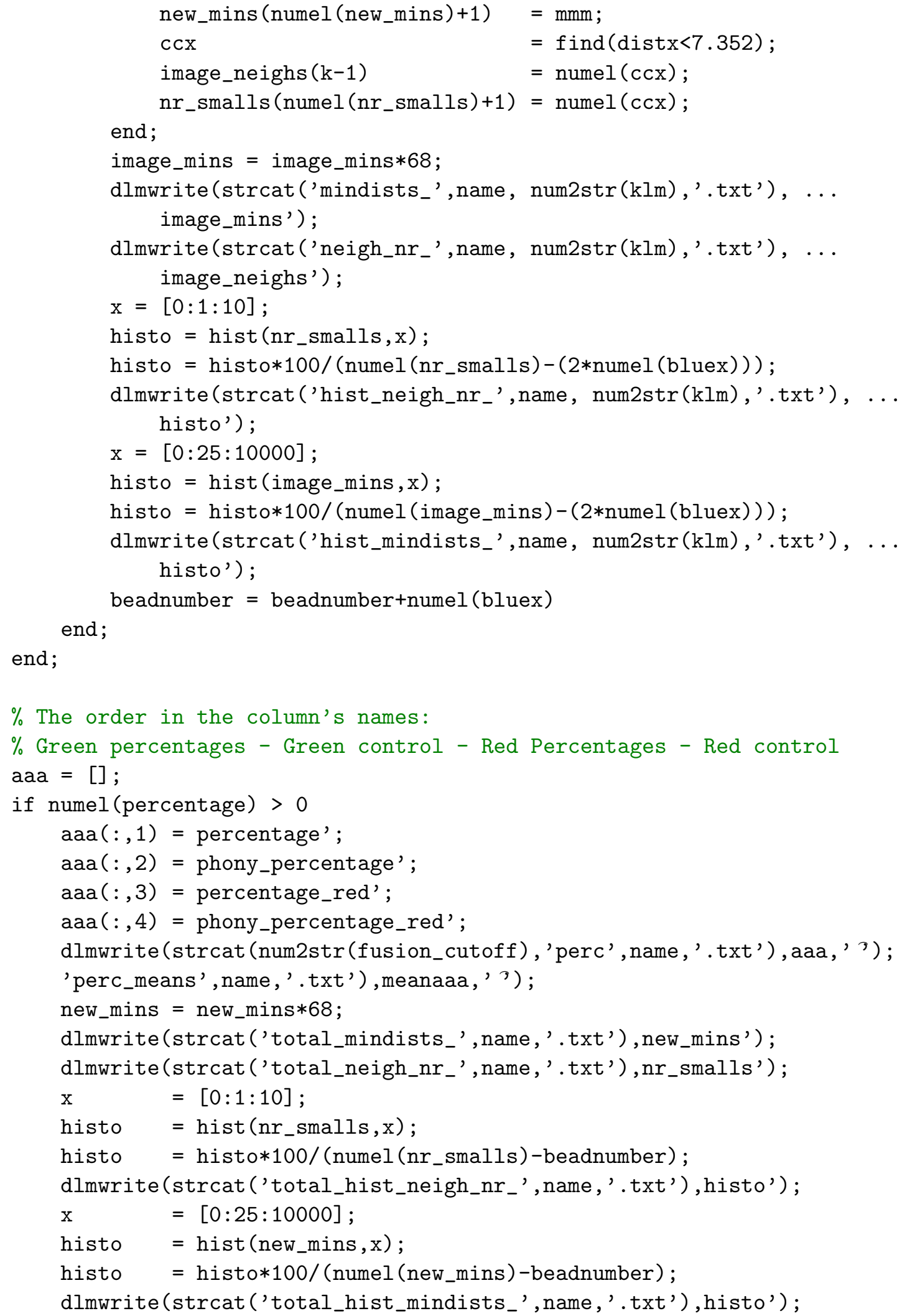




\section{BIBLIOGRAPHY}

Aalto, M.K., Ronne, H., and Keränen, S.: Yeast syntaxins Sso1p and Sso2p belong to a family of related membrane proteins that function in vesicular transport. EMBO J 12 (11): 4095-4104, 1993.

Advani, R.J., Bae, H.R., Bock, J.B., Chao, D.S., Doung, Y.C., Prekeris, R., Yoo, J.S., and Scheller, R.H.: Seven novel mammalian SNARE proteins localize to distinct membrane compartments. J Biol Chem 273 (17): 10.317-10.324, 1998.

Aguado, F., Maj, G., Ruiz-Montasell, B., Canals, J.M., Casanova, A., Marsal, J., and Blasi, J.: Expression of synaptosomal-associated protein SNAP-25 in endocrine anterior pituitary cells. Eur J Cell Biol 69 (4): 351-359, 1996.

Aikawa, Y., Lynch, K.L., Boswell, K.L., and Martin, T.F.J.: A second SNARE role for exocytic SNAP25 in endosome fusion. Mol Biol Cell 17 (5): 2113-2124, 2006.

Amessou, M., Fradagrada, A., Falguires, T., Lord, J.M., Smith, D.C., Roberts, L.M., Lamaze, C., and Johannes, L.: Syntaxin 16 and syntaxin 5 are required for efficient retrograde transport of several exogenous and endogenous cargo proteins. J Cell Sci 120 (Pt 8): 1457-1468, 2007.

Antonin, W., Holroyd, C., Tikkanen, R., Höning, S., and Jahn, R.: The R-SNARE endobrevin/VAMP-8 mediates homotypic fusion of early endosomes and late endosomes. Mol Biol Cell 11 (10): 3289-3298, 2000.

Antonin, W., Fasshauer, D., Becker, S., Jahn, R., and Schneider, T.R.: Crystal structure of the endosomal SNARE complex reveals common structural principles of all SNAREs. Nat Struct Biol 9 (2): 107-111, 2002. 
Aoyagi, K., Sugaya, T., Umeda, M., Yamamoto, S., Terakawa, S., and Takahashi, M.: The activation of exocytotic sites by the formation of phosphatidylinositol 4,5bisphosphate microdomains at syntaxin clusters. J Biol Chem 280 (17): 17.34617.352, 2005.

Arunachalam, L., Han, L., Tassew, N.G., He, Y., Wang, L., Xie, L., Fujita, Y., Kwan, E., Davletov, B., Monnier, P.P., Gaisano, H.Y., and Sugita, S.: Munc18-1 is critical for plasma membrane localization of syntaxin1 but not of SNAP-25 in PC12 cells. Mol Biol Cell 19 (2): 722-734, 2008.

Atlashkin, V., Kreykenbohm, V., Eskelinen, E.L., Wenzel, D., Fayyazi, A., and von Mollard, G.F.: Deletion of the SNARE vti1b in mice results in the loss of a single SNARE partner, syntaxin 8. Mol Cell Biol 23 (15): 5198-5207, 2003.

Bennett, M.K., Garca-Arrars, J.E., Elferink, L.A., Peterson, K., Fleming, A.M., Hazuka, C.D., and Scheller, R.H.: The syntaxin family of vesicular transport receptors. Cell 74 (5): 863-873, 1993.

Bentley, M., Liang, Y., Mullen, K., Xu, D., Sztul, E., and Hay, J.C.: SNARE status regulates tether recruitment and function in homotypic COPII vesicle fusion. $J$ Biol Chem 281 (50): 38.825-38.833, 2006.

Bergeland, T., Haugen, L., Landsverk, O.J.B., Stenmark, H., and Bakke, O.: Cellcycle-dependent binding kinetics for the early endosomal tethering factor EEA1. EMBO Rep 9 (2): 171-178, 2008.

Bethani, I., Lang, T., Geumann, U., Sieber, J.J., Jahn, R., and Rizzoli, S.O.: The specificity of SNARE pairing in biological membranes is mediated by both proofreading and spatial segregation. EMBO J 26 (17): 3981-3992, 2007.

Bhattacharya, S., Stewart, B.A., Niemeyer, B.A., Burgess, R.W., McCabe, B.D., Lin, P., Boulianne, G., O'Kane, C.J., and Schwarz, T.L.: Members of the synaptobrevin/vesicle-associated membrane protein (VAMP) family in Drosophila are functionally interchangeable in vivo for neurotransmitter release and cell viability. Proc Natl Acad Sci U S A 99 (21): 13.867-13.872, 2002. 
Block, M.R., Glick, B.S., Wilcox, C.A., Wieland, F.T., and Rothman, J.E.: Purification of an N-ethylmaleimide-sensitive protein catalyzing vesicular transport. Proc Natl Acad Sci U S A 85 (21): 7852-7856, 1988.

Bock, J.B., Matern, H.T., Peden, A.A., and Scheller, R.H.: A genomic perspective on membrane compartment organization. Nature 409 (6822): 839-841, 2001.

Borisovska, M., Zhao, Y., Tsytsyura, Y., Glyvuk, N., Takamori, S., Matti, U., Rettig, J., Südhof, T., and Bruns, D.: v-SNAREs control exocytosis of vesicles from priming to fusion. EMBO J 24 (12): 2114-2126, 2005.

Bose, A., Guilherme, A., Huang, S., Hubbard, A.C., Lane, C.R., Soriano, N.A., and Czech, M.P.: The v-SNARE Vtila regulates insulin-stimulated glucose transport and Acrp30 secretion in 3T3-L1 adipocytes. J Biol Chem 280 (44): 36.946-36.951, 2005.

Brandhorst, D., Zwilling, D., Rizzoli, S.O., Lippert, U., Lang, T., and Jahn, R.: Homotypic fusion of early endosomes: SNAREs do not determine fusion specificity. Proc Natl Acad Sci U S A 103 (8): 2701-2706, 2006.

Braun, S. and Jentsch, S.: SM-protein-controlled ER-associated degradation discriminates between different SNAREs. EMBO Rep 8 (12): 1176-1182, 2007.

Bryant, N.J. and James, D.E.: Vps45p stabilizes the syntaxin homologue Tlg2p and positively regulates SNARE complex formation. EMBO J 20 (13): 3380-3388, 2001.

Bryant, N.J. and James, D.E.: The Sec1p/Munc18 (SM) protein, Vps45p, cycles on and off membranes during vesicle transport. J Cell Biol 161 (4): 691-696, 2003.

Carr, C.M., Grote, E., Munson, M., Hughson, F.M., and Novick, P.J.: Sec1p binds to SNARE complexes and concentrates at sites of secretion. J Cell Biol 146 (2): 333-344, 1999.

Chamberlain, L.H., Burgoyne, R.D., and Gould, G.W.: SNARE proteins are highly enriched in lipid rafts in PC12 cells: implications for the spatial control of exocytosis. Proc Natl Acad Sci U S A 98 (10): 5619-5624, 2001. 
Choudhury, A., Marks, D.L., Proctor, K.M., Gould, G.W., and Pagano, R.E.: Regulation of caveolar endocytosis by syntaxin 6-dependent delivery of membrane components to the cell surface. Nat Cell Biol 8 (4): 317-328, 2006.

Christoforidis, S., McBride, H.M., Burgoyne, R.D., and Zerial, M.: The Rab5 effector EEA1 is a core component of endosome docking. Nature 397 (6720): 621-625, 1999.

Clary, D.O., Griff, I.C., and Rothman, J.E.: SNAPs, a family of NSF attachment proteins involved in intracellular membrane fusion in animals and yeast. Cell 61 (4): 709-721, 1990.

Coe, J.G., Lim, A.C., Xu, J., and Hong, W.: A role for Tlg1p in the transport of proteins within the Golgi apparatus of Saccharomyces cerevisiae. Mol Biol Cell 10 (7): 2407-2423, 1999.

Conner, S.D. and Schmid, S.L.: Regulated portals of entry into the cell. Nature 422 (6927): 37-44, 2003.

Cook, N.R., Row, P.E., and Davidson, H.W.: Lysosome associated membrane protein 1 (Lamp1) traffics directly from the TGN to early endosomes. Traffic $\mathbf{5}$ (9): 685-699, 2004.

D'Andrea-Merrins, M., Chang, L., Lam, A.D., Ernst, S.A., and Stuenkel, E.L.: Munc18c interaction with syntaxin 4 monomers and SNARE complex intermediates in GLUT4 vesicle trafficking. J Biol Chem 282 (22): 16.553-16.566, 2007.

Darsow, T., Rieder, S.E., and Emr, S.D.: A multispecificity syntaxin homologue, Vam3p, essential for autophagic and biosynthetic protein transport to the vacuole. J Cell Biol 138 (3): 517-529, 1997.

Delgado-Martnez, I., Nehring, R.B., and Srensen, J.B.: Differential abilities of SNAP-25 homologs to support neuronal function. J Neurosci 27 (35): 93809391, 2007.

Diaz, R., Mayorga, L., and Stahl, P.: In vitro fusion of endosomes following receptormediated endocytosis. J Biol Chem 263 (13): 6093-6100, 1988. 
Dietrich, L.E.P., Boeddinghaus, C., LaGrassa, T.J., and Ungermann, C.: Control of eukaryotic membrane fusion by N-terminal domains of SNARE proteins. Biochim Biophys Acta 1641 (2-3): 111-119, 2003.

Dulubova, I., Khvotchev, M., Liu, S., Huryeva, I., Südhof, T.C., and Rizo, J.: Munc18-1 binds directly to the neuronal SNARE complex. Proc Natl Acad Sci U S A 104 (8): 2697-2702, 2007.

Elferink, L.A., Trimble, W.S., and Scheller, R.H.: Two vesicle-associated membrane protein genes are differentially expressed in the rat central nervous system. J Biol Chem 264 (19): 11.061-11.064, 1989.

Fasshauer, D., Antonin, W., Margittai, M., Pabst, S., and Jahn, R.: Mixed and non-cognate SNARE complexes. Characterization of assembly and biophysical properties. J Biol Chem 274 (22): 15.440-15.446, 1999.

Fasshauer, D., Sutton, R.B., Brunger, A.T., and Jahn, R.: Conserved structural features of the synaptic fusion complex: SNARE proteins reclassified as Q- and R-SNAREs. Proc Natl Acad Sci U S A 95 (26): 15.781-15.786, 1998.

Fasshauer, D.: Structural insights into the SNARE mechanism. Biochim Biophys Acta 1641 (2-3): 87-97, 2003.

Fasshauer, D., Antonin, W., Subramaniam, V., and Jahn, R.: SNARE assembly and disassembly exhibit a pronounced hysteresis. Nat Struct Biol 9 (2): 144-151, 2002 .

Fries, E. and Rothman, J.E.: Transport of vesicular stomatitis virus glycoprotein in a cell-free extract. Proc Natl Acad Sci U S A 77 (7): 3870-3874, 1980.

Fujiwara, T., Mishima, T., Kofuji, T., Chiba, T., Tanaka, K., Yamamoto, A., and Akagawa, K.: Analysis of knock-out mice to determine the role of HPC-1/syntaxin 1A in expressing synaptic plasticity. J Neurosci 26 (21): 5767-5776, 2006.

Ganley, I.G., Espinosa, E., and Pfeffer, S.R.: A syntaxin 10-SNARE complex distinguishes two distinct transport routes from endosomes to the trans-Golgi in human cells. J Cell Biol 180 (1): 159-172, 2008. 
Gengyo-Ando, K., Kuroyanagi, H., Kobayashi, T., Murate, M., Fujimoto, K., Okabe, S., and Mitani, S.: The SM protein VPS-45 is required for RAB-5-dependent endocytic transport in Caenorhabditis elegans. EMBO Rep 8 (2): 152-157, 2007.

Gerst, J.E.: SNARE regulators: matchmakers and matchbreakers. Biochim Biophys Acta 1641 (2-3): 99-110, 2003.

Geumann, U., Barysch, S.V., Hoopmann, P., Jahn, R., and Rizzoli, S.O.: SNARE Function Is Not Involved in Early Endosome Docking. Mol Biol Cell 2008.

Gorvel, J.P., Chavrier, P., Zerial, M., and Gruenberg, J.: rab5 controls early endosome fusion in vitro. Cell 64 (5): 915-925, 1991.

Greene, L.A. and Tischler, A.S.: Establishment of a noradrenergic clonal line of rat adrenal pheochromocytoma cells which respond to nerve growth factor. Proc Natl Acad Sci U S A 73 (7): 2424-2428, 1976.

Grosshans, B.L., Ortiz, D., and Novick, P.: Rabs and their effectors: achieving specificity in membrane traffic. Proc Natl Acad Sci U S A 103 (32): 11.82111.827, 2006.

Gruenberg, J. and Howell, K.E.: Membrane traffic in endocytosis: insights from cell-free assays. Annu Rev Cell Biol 5: 453-481, 1989.

Hong, W.: SNAREs and traffic. Biochim Biophys Acta 1744 (2): 120-144, 2005.

Humeau, Y., Doussau, F., Grant, N.J., and Poulain, B.: How botulinum and tetanus neurotoxins block neurotransmitter release. Biochimie 82 (5): 427-446, 2000.

Huttner, W.B., Schiebler, W., Greengard, P., and Camilli, P.D.: Synapsin I (protein I), a nerve terminal-specific phosphoprotein. III. Its association with synaptic vesicles studied in a highly purified synaptic vesicle preparation. J Cell Biol 96 (5): 1374-1388, 1983.

Itin, C., Rancao, C., Nakajima, Y., and Pfeffer, S.R.: A novel assay reveals a role for soluble N-ethylmaleimide-sensitive fusion attachment protein in mannose 6phosphate receptor transport from endosomes to the trans Golgi network. J Biol Chem 272 (44): 27.737-27.744, 1997. 
Jahn, R. and Niemann, H.: Molecular mechanisms of clostridial neurotoxins. Ann N Y Acad Sci 733: 245-255, 1994.

Jahn, R. and Scheller, R.H.: SNAREs-engines for membrane fusion. Nat Rev Mol Cell Biol 7 (9): 631-643, 2006.

Kawasaki, F., Mattiuz, A.M., and Ordway, R.W.: Synaptic physiology and ultrastructure in comatose mutants define an in vivo role for NSF in neurotransmitter release. J Neurosci 18 (24): 10.241-10.249, 1998.

Khvotchev, M., Dulubova, I., Sun, J., Dai, H., Rizo, J., and Südhof, T.C.: Dual modes of Munc18-1/SNARE interactions are coupled by functionally critical binding to syntaxin-1 N terminus. J Neurosci 27 (45): 12.147-12.155, 2007.

Klenchin, V.A., Kowalchyk, J.A., and Martin, T.F.: Large dense-core vesicle exocytosis in PC12 cells. Methods 16 (2): 204-208, 1998.

Kosodo, Y., Noda, Y., Adachi, H., and Yoda, K.: Binding of Sly1 to Sed5 enhances formation of the yeast early Golgi SNARE complex. J Cell Sci 115 (Pt 18): 3683-3691, 2002.

Kreykenbohm, V., Wenzel, D., Antonin, W., Atlachkine, V., and von Mollard, G.F.: The SNAREs vtila and vtilb have distinct localization and SNARE complex partners. Eur J Cell Biol 81 (5): 273-280, 2002.

Lang, T., Bruns, D., Wenzel, D., Riedel, D., Holroyd, P., Thiele, C., and Jahn, R.: SNAREs are concentrated in cholesterol-dependent clusters that define docking and fusion sites for exocytosis. EMBO J 20 (9): 2202-2213, 2001.

Latham, C.F., Lopez, J.A., Hu, S.H., Gee, C.L., Westbury, E., Blair, D.H., Armishaw, C.J., Alewood, P.F., Bryant, N.J., James, D.E., and Martin, J.L.: Molecular dissection of the Munc18c/syntaxin4 interaction: implications for regulation of membrane trafficking. Traffic 7 (10): 1408-1419, 2006.

Lencer, W.I. and Tsai, B.: The intracellular voyage of cholera toxin: going retro. Trends Biochem Sci 28 (12): 639-645, 2003. 
Link, E., McMahon, H., von Mollard, G.F., Yamasaki, S., Niemann, H., Südhof, T.C., and Jahn, R.: Cleavage of cellubrevin by tetanus toxin does not affect fusion of early endosomes. J Biol Chem 268 (25): 18.423-18.426, 1993.

Liu, Y. and Barlowe, C.: Analysis of Sec22p in endoplasmic reticulum/Golgi transport reveals cellular redundancy in SNARE protein function. Mol Biol Cell 13 (9): 3314-3324, 2002.

Low, S.H., Vasanji, A., Nanduri, J., He, M., Sharma, N., Koo, M., Drazba, J., and Weimbs, T.: Syntaxins 3 and 4 are concentrated in separate clusters on the plasma membrane before the establishment of cell polarity. Mol Biol Cell 17 (2): 977-989, 2006.

Malsam, J., Kreye, S., and Söllner, T.H.: Membrane fusion: SNAREs and regulation. Cell Mol Life Sci 65 (18): 2814-2832, 2008.

Marsal, J., Ruiz-Montasell, B., Blasi, J., Moreira, J.E., Contreras, D., Sugimori, M., and Llins, R.: Block of transmitter release by botulinum C1 action on syntaxin at the squid giant synapse. Proc Natl Acad Sci U S A 94 (26): 14.871-14.876, 1997.

Maxfield, F.R. and McGraw, T.E.: Endocytic recycling. Nat Rev Mol Cell Biol 5 (2): 121-132, 2004.

McBride, H.M., Rybin, V., Murphy, C., Giner, A., Teasdale, R., and Zerial, M.: Oligomeric complexes link Rab5 effectors with NSF and drive membrane fusion via interactions between EEA1 and syntaxin 13. Cell 98 (3): 377-386, 1999.

McNew, J.A., Parlati, F., Fukuda, R., Johnston, R.J., Paz, K., Paumet, F., Söllner, T.H., and Rothman, J.E.: Compartmental specificity of cellular membrane fusion encoded in SNARE proteins. Nature 407 (6801): 153-159, 2000.

McNew, J.A., Sogaard, M., Lampen, N.M., Machida, S., Ye, R.R., Lacomis, L., Tempst, P., Rothman, J.E., and Söllner, T.H.: Ykt6p, a prenylated SNARE essential for endoplasmic reticulum-Golgi transport. J Biol Chem 272 (28): 17.77617.783, 1997. 
Medine, C.N., Rickman, C., Chamberlain, L.H., and Duncan, R.R.: Munc18-1 prevents the formation of ectopic SNARE complexes in living cells. J Cell Sci 120 (Pt 24): 4407-4415, 2007.

Mellman, I.: Endocytosis and molecular sorting. Annu Rev Cell Dev Biol 12: 575625, 1996.

Miller, S.E., Collins, B.M., McCoy, A.J., Robinson, M.S., and Owen, D.J.: A SNARE-adaptor interaction is a new mode of cargo recognition in clathrin-coated vesicles. Nature 450 (7169): 570-574, 2007.

Mills, I.G., Urb, S., and Clague, M.J.: Relationships between EEA1 binding partners and their role in endosome fusion. J Cell Sci 114 (Pt 10): 1959-1965, 2001.

von Mollard, G.F., Stahl, B., Walch-Solimena, C., Takei, K., Daniels, L., Khoklatchev, A., Camilli, P.D., Südhof, T.C., and Jahn, R.: Localization of Rab5 to synaptic vesicles identifies endosomal intermediate in synaptic vesicle recycling pathway. Eur J Cell Biol 65 (2): 319-326, 1994.

Montecucco, C. and Schiavo, G.: Mechanism of action of tetanus and botulinum neurotoxins. Mol Microbiol 13 (1): 1-8, 1994.

Montecucco, C., Schiavo, G., and Pantano, S.: SNARE complexes and neuroexocytosis: how many, how close? Trends Biochem Sci 30 (7): 367-372, 2005.

Mukherjee, S., Ghosh, R.N., and Maxfield, F.R.: Endocytosis. Physiol Rev 77 (3): 759-803, 1997.

Nielsen, E., Christoforidis, S., Uttenweiler-Joseph, S., Miaczynska, M., Dewitte, F., Wilm, M., Hoflack, B., and Zerial, M.: Rabenosyn-5, a novel Rab5 effector, is complexed with hVPS45 and recruited to endosomes through a FYVE finger domain. J Cell Biol 151 (3): 601-612, 2000.

Novick, P., Field, C., and Schekman, R.: Identification of 23 complementation groups required for post-translational events in the yeast secretory pathway. Cell 21 (1): 205-215, 1980. 
Novick, P. and Schekman, R.: Secretion and cell-surface growth are blocked in a temperature-sensitive mutant of Saccharomyces cerevisiae. Proc Natl Acad Sci U $S A 76$ (4): 1858-1862, 1979.

Okayama, M., Arakawa, T., Mizoguchi, I., Tajima, Y., and Takuma, T.: SNAP-23 is not essential for constitutive exocytosis in HeLa cells. FEBS Lett 581 (24): 4583-4588, 2007.

Palade, G.: Intracellular aspects of the process of protein synthesis. Science 189 (4200): 347-358, 1975.

Paolo, G.D. and Camilli, P.D.: Phosphoinositides in cell regulation and membrane dynamics. Nature 443 (7112): 651-657, 2006.

Parlati, F., McNew, J.A., Fukuda, R., Miller, R., Söllner, T.H., and Rothman, J.E.: Topological restriction of SNARE-dependent membrane fusion. Nature 407 (6801): 194-198, 2000.

Paumet, F., Rahimian, V., and Rothman, J.E.: The specificity of SNARE-dependent fusion is encoded in the SNARE motif. Proc Natl Acad Sci U S A 101 (10): 33763380, 2004.

Peng, R. and Gallwitz, D.: Sly1 protein bound to Golgi syntaxin Sed5p allows assembly and contributes to specificity of SNARE fusion complexes. J Cell Biol 157 (4): 645-655, 2002.

Pfeffer, S.R.: Rab GTPases: specifying and deciphering organelle identity and function. Trends Cell Biol 11 (12): 487-491, 2001.

Pfeffer, S.R.: Unsolved mysteries in membrane traffic. Annu Rev Biochem 76: 629$645,2007$.

Predescu, S.A., Predescu, D.N., Shimizu, K., Klein, I.K., and Malik, A.B.: Cholesterol-dependent syntaxin-4 and SNAP-23 clustering regulates caveolar fusion with the endothelial plasma membrane. J Biol Chem 280 (44): 37.13037.138, 2005. 
Prekeris, R., Klumperman, J., Chen, Y.A., and Scheller, R.H.: Syntaxin 13 mediates cycling of plasma membrane proteins via tubulovesicular recycling endosomes. $J$ Cell Biol 143 (4): 957-971, 1998.

Price, A., Seals, D., Wickner, W., and Ungermann, C.: The docking stage of yeast vacuole fusion requires the transfer of proteins from a cis-SNARE complex to a Rab/Ypt protein. J Cell Biol 148 (6): 1231-1238, 2000.

Proctor, K.M., Miller, S.C.M., Bryant, N.J., and Gould, G.W.: Syntaxin 16 controls the intracellular sequestration of GLUT4 in 3T3-L1 adipocytes. Biochem Biophys Res Commun 347 (2): 433-438, 2006.

Protopopov, V., Govindan, B., Novick, P., and Gerst, J.E.: Homologs of the synaptobrevin/VAMP family of synaptic vesicle proteins function on the late secretory pathway in S. cerevisiae. Cell 74 (5): 855-861, 1993.

Pryor, P.R., Jackson, L., Gray, S.R., Edeling, M.A., Thompson, A., Sanderson, C.M., Evans, P.R., Owen, D.J., and Luzio, J.P.: Molecular basis for the sorting of the SNARE VAMP7 into endocytic clathrin-coated vesicles by the ArfGAP Hrb. Cell 134 (5): 817-827, 2008.

Rickman, C., Meunier, F.A., Binz, T., and Davletov, B.: High affinity interaction of syntaxin and SNAP-25 on the plasma membrane is abolished by botulinum toxin E. J Biol Chem 279 (1): 644-651, 2004.

Riedel, D., Antonin, W., Fernandez-Chacon, R., de Toledo, G.A., Jo, T., Geppert, M., Valentijn, J.A., Valentijn, K., Jamieson, J.D., Südhof, T.C., and Jahn, R.: Rab3D is not required for exocrine exocytosis but for maintenance of normally sized secretory granules. Mol Cell Biol 22 (18): 6487-6497, 2002.

Riederer, M.A., Soldati, T., Shapiro, A.D., Lin, J., and Pfeffer, S.R.: Lysosome biogenesis requires Rab9 function and receptor recycling from endosomes to the trans-Golgi network. J Cell Biol 125 (3): 573-582, 1994.

Rizo, J., Chen, X., and Ara, D.: Unraveling the mechanisms of synaptotagmin and 
SNARE function in neurotransmitter release. Trends Cell Biol 16 (7): 339-350, 2006.

Rizzoli, S.O., Bethani, I., Zwilling, D., Wenzel, D., Siddiqui, T.J., Brandhorst, D., and Jahn, R.: Evidence for early endosome-like fusion of recently endocytosed synaptic vesicles. Traffic 7 (9): 1163-1176, 2006.

Rybin, V., Ullrich, O., Rubino, M., Alexandrov, K., Simon, I., Seabra, M.C., Goody, R., and Zerial, M.: GTPase activity of Rab5 acts as a timer for endocytic membrane fusion. Nature 383 (6597): 266-269, 1996.

Scales, S.J., Chen, Y.A., Yoo, B.Y., Patel, S.M., Doung, Y.C., and Scheller, R.H.: SNAREs contribute to the specificity of membrane fusion. Neuron 26 (2): 457464, 2000.

Schägger, H. and von Jagow, G.: Tricine-sodium dodecyl sulfate-polyacrylamide gel electrophoresis for the separation of proteins in the range from 1 to $100 \mathrm{kDa}$. Anal Biochem 166 (2): 368-379, 1987.

Schoch, S., Dek, F., Königstorfer, A., Mozhayeva, M., Sara, Y., Südhof, T.C., and Kavalali, E.T.: SNARE function analyzed in synaptobrevin/VAMP knockout mice. Science 294 (5544): 1117-1122, 2001.

Scott, B.L., Komen, J.S.V., Irshad, H., Liu, S., Wilson, K.A., and McNew, J.A.: Sec1p directly stimulates SNARE-mediated membrane fusion in vitro. J Cell Biol 167 (1): 75-85, 2004.

Seals, D.F., Eitzen, G., Margolis, N., Wickner, W.T., and Price, A.: A Ypt/Rab effector complex containing the Sec1 homolog Vps33p is required for homotypic vacuole fusion. Proc Natl Acad Sci U S A 97 (17): 9402-9407, 2000.

Shen, J., Tareste, D.C., Paumet, F., Rothman, J.E., and Melia, T.J.: Selective activation of cognate SNAREpins by Sec1/Munc18 proteins. Cell 128 (1): 183$195,2007$. 
Shorter, J., Beard, M.B., Seemann, J., Dirac-Svejstrup, A.B., and Warren, G.: Sequential tethering of Golgins and catalysis of SNAREpin assembly by the vesicletethering protein p115. J Cell Biol 157 (1): 45-62, 2002.

Sieber, J.J., Willig, K.I., Heintzmann, R., Hell, S.W., and Lang, T.: The SNARE motif is essential for the formation of syntaxin clusters in the plasma membrane. Biophys J 90 (8): 2843-2851, 2006.

Sieber, J.J., Willig, K.I., Kutzner, C., Gerding-Reimers, C., Harke, B., Donnert, G., Rammner, B., Eggeling, C., Hell, S.W., Grubmüller, H., and Lang, T.: Anatomy and dynamics of a supramolecular membrane protein cluster. Science 317 (5841): 1072-1076, 2007.

Simonsen, A., Gaullier, J.M., D'Arrigo, A., and Stenmark, H.: The Rab5 effector EEA1 interacts directly with syntaxin-6. J Biol Chem 274 (41): 28.857-28.860, 1999.

Söllner, T., Whiteheart, S.W., Brunner, M., Erdjument-Bromage, H., Geromanos, S., Tempst, P., and Rothman, J.E.: SNAP receptors implicated in vesicle targeting and fusion. Nature 362 (6418): 318-324, 1993.

Steegmaier, M., Klumperman, J., Foletti, D.L., Yoo, J.S., and Scheller, R.H.: Vesicle-associated membrane protein 4 is implicated in trans-Golgi network vesicle trafficking. Mol Biol Cell 10 (6): 1957-1972, 1999.

Steinman, R.M., Brodie, S.E., and Cohn, Z.A.: Membrane flow during pinocytosis. A stereologic analysis. J Cell Biol 68 (3): 665-687, 1976.

Stenmark, H., Parton, R.G., Steele-Mortimer, O., Lütcke, A., Gruenberg, J., and Zerial, M.: Inhibition of rab5 GTPase activity stimulates membrane fusion in endocytosis. EMBO J 13 (6): 1287-1296, 1994.

Sudhof, T.C.: The synaptic vesicle cycle. Annu Rev Neurosci 27: 509-547, 2004.

Suga, K., Hattori, H., Saito, A., and Akagawa, K.: RNA interference-mediated silencing of the syntaxin 5 gene induces Golgi fragmentation but capable of transporting vesicles. FEBS Lett 579 (20): 4226-4234, 2005. 
Sun, W., Yan, Q., Vida, T.A., and Bean, A.J.: Hrs regulates early endosome fusion by inhibiting formation of an endosomal SNARE complex. J Cell Biol 162 (1): 125-137, 2003.

Sutton, R.B., Fasshauer, D., Jahn, R., and Brunger, A.T.: Crystal structure of a SNARE complex involved in synaptic exocytosis at $2.4 \mathrm{~A}$ resolution. Nature 395 (6700): 347-353, 1998.

Sztul, E. and Lupashin, V.: Role of tethering factors in secretory membrane traffic. Am J Physiol Cell Physiol 290 (1): C11-C26, 2006.

Takamori, S., Holt, M., Stenius, K., Lemke, E.A., Grnborg, M., Riedel, D., Urlaub, H., Schenck, S., Brügger, B., Ringler, P., Müller, S.A., Rammner, B., Gräter, F., Hub, J.S., Groot, B.L.D., Mieskes, G., Moriyama, Y., Klingauf, J., Grubmüller, H., Heuser, J., Wieland, F., and Jahn, R.: Molecular anatomy of a trafficking organelle. Cell 127 (4): 831-846, 2006.

Tang, B.L., Tan, A.E., Lim, L.K., Lee, S.S., Low, D.Y., and Hong, W.: Syntaxin 12, a member of the syntaxin family localized to the endosome. J Biol Chem 273 (12): 6944-6950, 1998.

Tang, J., Maximov, A., Shin, O.H., Dai, H., Rizo, J., and Südhof, T.C.: A complexin/synaptotagmin 1 switch controls fast synaptic vesicle exocytosis. Cell 126 (6): 1175-1187, 2006.

Togneri, J., Cheng, Y.S., Munson, M., Hughson, F.M., and Carr, C.M.: Specific SNARE complex binding mode of the Sec1/Munc-18 protein, Sec1p. Proc Natl Acad Sci U S A 103 (47): 17.730-17.735, 2006.

Tsui, M.M. and Banfield, D.K.: Yeast Golgi SNARE interactions are promiscuous. J Cell Sci 113 ( Pt 1): 145-152, 2000.

Ungermann, C., Price, A., and Wickner, W.: A new role for a SNARE protein as a regulator of the Ypt7/Rab-dependent stage of docking. Proc Natl Acad Sci U S A 97 (16): 8889-8891, 2000. 
Verhage, M., Maia, A.S., Plomp, J.J., Brussaard, A.B., Heeroma, J.H., Vermeer, H., Toonen, R.F., Hammer, R.E., van den Berg, T.K., Missler, M., Geuze, H.J., and Südhof, T.C.: Synaptic assembly of the brain in the absence of neurotransmitter secretion. Science 287 (5454): 864-869, 2000.

Voets, T., Toonen, R.F., Brian, E.C., de Wit, H., Moser, T., Rettig, J., Südhof, T.C., Neher, E., and Verhage, M.: Munc18-1 promotes large dense-core vesicle docking. Neuron 31 (4): 581-591, 2001.

Walch-Solimena, C., Blasi, J., Edelmann, L., Chapman, E.R., von Mollard, G.F., and Jahn, R.: The t-SNAREs syntaxin 1 and SNAP-25 are present on organelles that participate in synaptic vesicle recycling. J Cell Biol 128 (4): 637-645, 1995.

Wang, C.C., Ng, C.P., Lu, L., Atlashkin, V., Zhang, W., Seet, L.F., and Hong, W.: A role of VAMP8/endobrevin in regulated exocytosis of pancreatic acinar cells. Dev Cell 7 (3): 359-371, 2004.

Wang, L., Merz, A.J., Collins, K.M., and Wickner, W.: Hierarchy of protein assembly at the vertex ring domain for yeast vacuole docking and fusion. J Cell Biol 160 (3): 365-374, 2003.

Wang, Y., Tai, G., Lu, L., Johannes, L., Hong, W., and Tang, B.L.: Trans-Golgi network syntaxin 10 functions distinctly from syntaxins 6 and 16. Mol Membr Biol 22 (4): 313-325, 2005.

Washbourne, P., Thompson, P.M., Carta, M., Costa, E.T., Mathews, J.R., LopezBendit, G., Molnr, Z., Becher, M.W., Valenzuela, C.F., Partridge, L.D., and Wilson, M.C.: Genetic ablation of the t-SNARE SNAP-25 distinguishes mechanisms of neuroexocytosis. Nat Neurosci 5 (1): 19-26, 2002.

Watson, R.T., Hou, J.C., and Pessin, J.E.: Recycling of IRAP from the plasma membrane back to the insulin-responsive compartment requires the Q-SNARE syntaxin 6 but not the GGA clathrin adaptors. J Cell Sci 121 (Pt 8): 1243-1251, 2008. 
van Weering, J.R.T., Toonen, R.F., and Verhage, M.: The role of Rab3a in secretory vesicle docking requires association/dissociation of guanidine phosphates and Munc18-1. PLoS ONE 2 (7): e616, 2007.

Wendler, F. and Tooze, S.: Syntaxin 6: the promiscuous behaviour of a SNARE protein. Traffic 2 (9): 606-611, 2001.

Wickner, W. and Schekman, R.: Membrane fusion. Nat Struct Mol Biol 15 (7): 658-664, 2008.

Yang, B., Gonzalez, L., Prekeris, R., Steegmaier, M., Advani, R.J., and Scheller, R.H.: SNARE interactions are not selective. Implications for membrane fusion specificity. J Biol Chem 274 (9): 5649-5653, 1999.

Yang, C., Mora, S., Ryder, J.W., Coker, K.J., Hansen, P., Allen, L.A., and Pessin, J.E.: VAMP3 null mice display normal constitutive, insulin- and exerciseregulated vesicle trafficking. Mol Cell Biol 21 (5): 1573-1580, 2001.

Zerial, M. and McBride, H.: Rab proteins as membrane organizers. Nat Rev Mol Cell Biol 2 (2): 107-117, 2001.

Zilly, F.E., Srensen, J.B., Jahn, R., and Lang, T.: Munc18-bound syntaxin readily forms SNARE complexes with synaptobrevin in native plasma membranes. PLoS Biol 4 (10): e330, 2006.

Zwilling, D., Cypionka, A., Pohl, W.H., Fasshauer, D., Walla, P.J., Wahl, M.C., and Jahn, R.: Early endosomal SNAREs form a structurally conserved SNARE complex and fuse liposomes with multiple topologies. EMBO J 26 (1): 9-18, 2007. 


\section{Curriculum Vitæ}

\section{Personal Details}

Name

Date of birth

Place of birth

Address

Phone

E-mail

Graduate Studies

since 2006

Academic Degree

Master's Thesis

2006

\section{Undergraduate Studies}

$2004-2005$

Diploma Thesis

2004
Ioanna Bethani

April $13^{\text {th }} 1982$

Athens, Greece

Nikolaistr. 28, 37073 Göttingen, Germany

$+49-551-9954090$

ibethan@gwdg.de

\section{Education}

International MSc/PhD/MD-PhD Program Neurosciences,

Department of Neurobiology,

Max-Planck-Institute for Biophysical Chemistry,

Supervisor: Prof. R. Jahn

Master of Science

Characterization of SNARE interactions

from the early endosomal compartment

Department of Neurobiology,

Max-Planck-Institute for Biophysical Chemistry,

Supervisor: Prof. R. Jahn

International MSc/PhD/MD-PhD Program Neurosciences,

Max-Planck-Research School for Neurosciences, Göttingen

Investigating the mechanisms of Amyloid Precursor Protein (APP)

regulation by neuronal proteins Fe65, X11a and mDab1

Department of Physiology,

National and Kapodistrian University of Athens, Greece,

Supervisor: Prof. S. Efthimiopoulos 


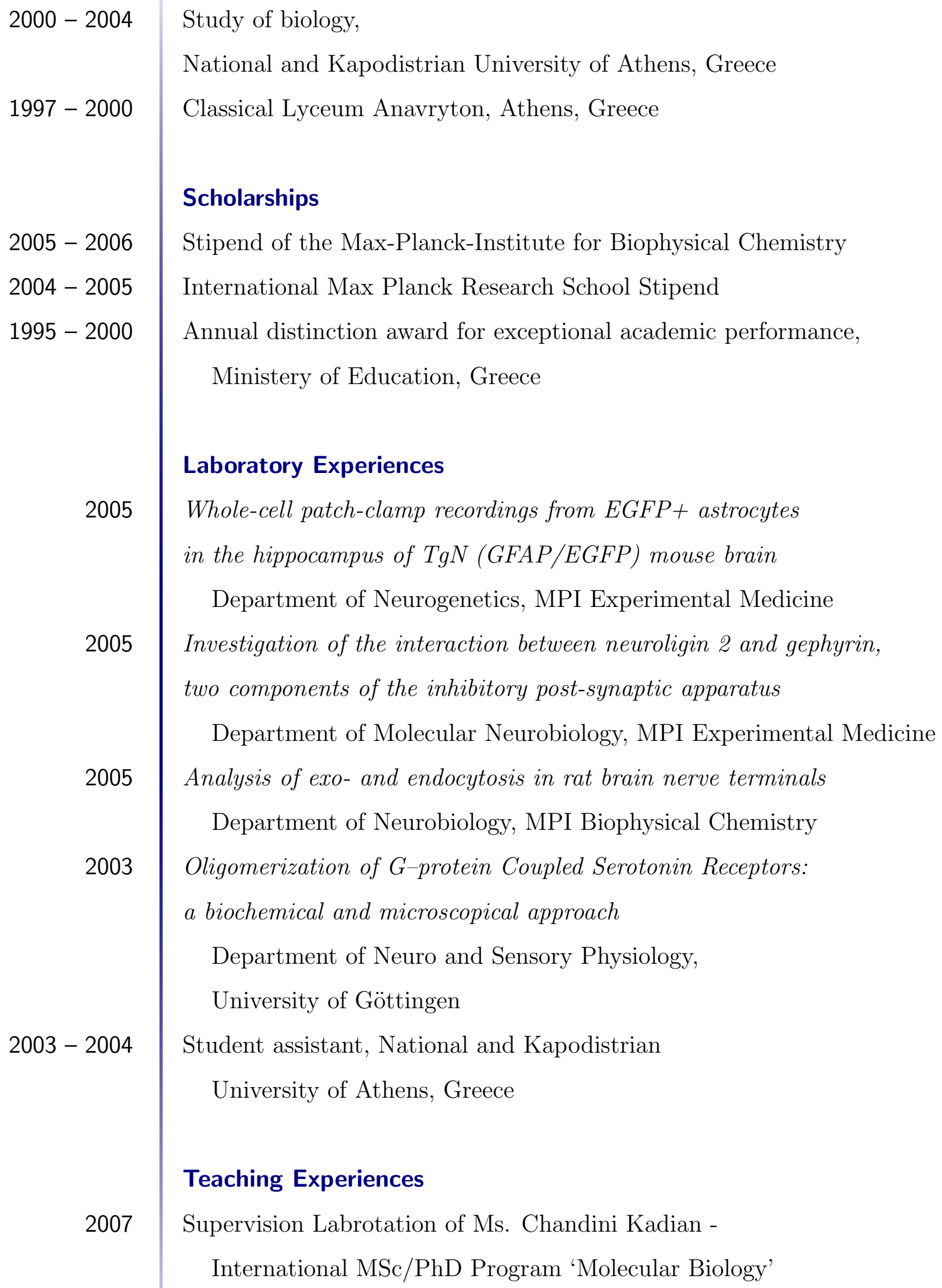

Scholarships

2005 - 2006 Stipend of the Max-Planck-Institute for Biophysical Chemistry

2004 - 2005 International Max Planck Research School Stipend

1995 - 2000 Annual distinction award for exceptional academic performance,

Ministery of Education, Greece

\section{Laboratory Experiences}

Whole-cell patch-clamp recordings from EGFP+ astrocytes in the hippocampus of $\operatorname{TgN}$ (GFAP/EGFP) mouse brain

Department of Neurogenetics, MPI Experimental Medicine Investigation of the interaction between neuroligin 2 and gephyrin, two components of the inhibitory post-synaptic apparatus

Department of Molecular Neurobiology, MPI Experimental Medicine Analysis of exo- and endocytosis in rat brain nerve terminals

Department of Neurobiology, MPI Biophysical Chemistry

2003 Oligomerization of G-protein Coupled Serotonin Receptors:

a biochemical and microscopical approach

Department of Neuro and Sensory Physiology,

University of Göttingen

2003 - $2004 \quad$ Student assistant, National and Kapodistrian

University of Athens, Greece

\section{Teaching Experiences}

2007 Supervision Labrotation of Ms. Chandini Kadian -

International MSc/PhD Program 'Molecular Biology' 
2005 - 2009 Tutorials on the lectures for 'Protein Sorting and Processing',

2006 - 2008 Tutor for the European Initiative for Communicators of Science (EICOS)

2008 Method course on the functional analysis of early endosomal fusion

(Graduate School for Neurosciences and Molecular Biosciences,

Göttingen, Germany)

\section{Conferencess}

The 48th Annual Meeting of the American Society for Cell Biology,

San Francisco, USA

Poster Presentation

The Golgi Meeting 2008, Pavia, Italy

Poster Presentation

SFB Retreat, Schloss Ringberg, Kreuth, Germany

Oral Presentation

2nd International PhD Student Symposium: 'Neurizons',

Göttingen, Germany

Poster Presentation

5th Forum of European Neuroscience, Vienna, Austria

Poster Presentation

26th Meeting of the Hellenic Society of Biology, Volos, Greece

Poster Presentation

\section{Activities and Interests}

Playing classical guitar, member of amateur theater groups,

felting, reading, traveling. 


\section{List of Publications}

Bethani I., Werner A., Kadian C., Geumann U., Jahn R., Rizzoli S.O.: Endosomal fusion upon SNARE knock-down is maintained by residual SNARE activity and enhanced docking. Submitted.

Halemani N., Bethani I., Rizzoli S.O., Lang T.: Structure and dynamics of a two-helix-SNARE-complex in live cells. Submitted.

Parisiadou L., Bethani I., Michaki V., Krousti K., Rapti G., Efthimiopoulos S.: Homer2 and Homer3 interact with amyloid precursor protein and inhibit Abeta production. Neurobiol Dis. 30 (3): 353 - 64, 2008.

Bethani I., Lang T., Geumann U., Sieber J.J., Jahn R., Rizzoli S.O.: The specificity of SNARE pairing in biological membranes is mediated by both proof-reading and spatial segregation. EMBO J. 26 (17): 3981 - 92, 2007.

Rizzoli S.O., Bethani I., Zwilling D., Wenzel D., Siddiqui T.J., Brandhorst D., Jahn R.: Evidence for early endosome-like fusion of recently endocytosed synaptic vesicles. Traffic. 7 (9): $1163-76,2006$.

Parisiadou L., Fassa A., Fotinopoulou A., Bethani I., Efthimiopoulos S.: Presenilin 1 and cadherins: stabilization of cell-cell adhesion and proteolysis-dependent regulation of transcription. Neurodegener Dis. 1 (4-5): 184 - 91, 2004. 